\title{
ARTICLE
}

Cite this: DOI: 10.1039/x0xx00000x

\section{Sticking and patching: tuning and anchoring cyclometallated ruthenium(II) complexes}

\author{
Cathrin D. Ertl, ${ }^{a}$ Daniel P. Ris, ${ }^{a}$ Stefan C. Meier, ${ }^{b}$ Edwin C. Constable ${ }^{a}$, Catherine \\ E. Housecroft, ${ }^{* a}$ Markus Neuburger ${ }^{a}$ and Jennifer A. Zampese ${ }^{a}$
}

Received 00th January 2012,

Accepted 00th January 2012

DOI: $10.1039 / \times 0 \times x 00000 x$

www.rsc.org/
This paper is dedicated to the memory of Kenneth (Ken) Wade, a pioneer in his field, and whose creative thinking and ability to encourage his students to think set him apart in the scientific world.

\begin{abstract}
A series of $\left[\mathrm{Ru}(\mathrm{bpy})_{2}(\mathrm{C} \wedge \mathrm{N})\right]\left[\mathrm{PF}_{6}\right](\mathrm{HC} \wedge \mathrm{N}=2$-phenylpyridine derivative $)$ complexes functionalized in the cyclometallating $\mathrm{C} \wedge \mathrm{N}$ phenyl ring with $\mathrm{F}, \mathrm{Me}, \mathrm{OMe}, \mathrm{CO}_{2} \mathrm{Me}, \mathrm{S}^{\mathrm{t}} \mathrm{Bu}, \mathrm{SO}_{2} \mathrm{Me}$ (ligands $\mathrm{H1}-\mathrm{H} 6$ ) or in the $\mathrm{C} \wedge \mathrm{N}$ pyridine ring with $4-\mathrm{CO}_{2} \mathrm{Me}$ or $4-\mathrm{C}_{6} \mathrm{H}_{4} \mathrm{P}(\mathrm{O})(\mathrm{OEt})_{2}$ substituents (ligands $\mathrm{H} \mathbf{7}$ or $\mathrm{H} 9$ ) have been prepared and characterized; representative crystal structures confirm the structural features of the complexes. When the $\mathrm{C} \wedge \mathrm{N}$ ligand contains a $\mathrm{CO}_{2} \mathrm{H}$ substituent (ligand $\mathrm{H}_{2} 8$ ), deprotonation in addition to cyclometallation occurs to give a neutral, zwitter-ionic complex $\left[R u(b p y)_{2}(8)\right]$. The synthesis of the cationic complexes requires addition of a silver(l) salt $\left(\mathrm{AgPF}_{6}\right)$ to abstract $\mathrm{Cl}^{-}$from cis- $\left[\mathrm{Ru}(\mathrm{bpy})_{2} \mathrm{Cl}_{2}\right]$ and ${ }^{1} \mathrm{H}$ NMR spectroscopic data are consistent with interactions between $\mathrm{Ag}^{+}$and the coordinated $\mathrm{C} \wedge \mathrm{N}$ ligand in $\left[R u(b p y)_{2}(C \wedge N)\right]^{+}$. The absorption spectra of $\left[R u(b p y)_{2}(C \wedge N)\right]\left[P F_{6}\right](C \wedge N=1-6)$ are similar, but the introduction of the anchoring domains in $\left[R u(b p y)_{2}(C \wedge N)\right]\left[P F_{6}\right]$ with $C \wedge N=\mathbf{7}$ or 9 enhances the absorption response; the greatest influence is observed in $\left[\mathrm{Ru}(\mathrm{bpy})_{2}(\mathbf{9})\right]^{+}$with the introduction of the 4$\mathrm{C}_{6} \mathrm{H}_{4} \mathrm{P}(\mathrm{O})(\mathrm{OEt})_{2}$ substituent. Trends in emission and electrochemical behaviours of the complexes are interpreted in terms of the influence of the electronic properties of the $\mathrm{C} \wedge \mathrm{N}$ ligand substituents on the energies of the HOMO which is localized on the $C \wedge N$ ligand and $\mathrm{Ru}$ centre. This study provides an optimized synthetic route to the phosphonate ester derivative $\left[\mathrm{Ru}(\mathrm{bpy})_{2}(\mathbf{9})\right]\left[\mathrm{PF}_{6}\right]$, designed to anchor to a semiconductor surface; this complex also exhibits the most favourable absorption properties among the complexes studied.
\end{abstract}

\section{Introduction}

The tris-chelate $\left[\mathrm{Ru}(\mathrm{bpy})_{2}(\mathrm{ppy})\right]^{+}$(bpy $=2,2^{\prime}$-bipyridine, Hppy $=2$-phenylpyridine) is an archetype cyclometallated analogue ${ }^{1,2}$ of $\left[\mathrm{Ru}(\mathrm{bpy})_{3}\right]^{2+}$. Over the last few years, there has been a renewed interest in cyclometallated ruthenium(II) complexes in the context of their application in n-type dye-sensitized solar cells (DSCs). The replacement of thiocyanate ligands in conventional ruthenium(II) sensitizers by cyclometallating ligands ${ }^{3,4,5}$ was first established by van Koten and coworkers ${ }^{6}$ using a $\mathrm{C}^{\wedge} \mathrm{N}^{\wedge} \mathrm{N}$ donor set. In 2009, Grätzel and coworkers reported a $10.1 \%$ global efficiency for a DSC containing $\left[\mathrm{Ru}(\mathrm{dcbpy})_{2}\left(\mathrm{~F}_{2} \mathrm{ppy}\right)\right]^{+} \quad\left(\mathrm{dcbpy}=2,2^{\prime}\right.$-bipyridine-4,4'dicarboxylic acid, $\mathrm{H}\left(\mathrm{F}_{2}\right.$ ppy $)=2-(2,4$-difluorophenyl $)$ pyridine $){ }^{7}$ Replacing thiocyanate by an anionic $\mathrm{C}^{\wedge} \mathrm{N}$ ligand provides a means of tuning the HOMO-LUMO properties of the dyes. The HOMO of a $\left[\mathrm{Ru}\left(\mathrm{N}^{\wedge} \mathrm{N}\right)_{2}\left(\mathrm{C}^{\wedge} \mathrm{N}\right)\right]^{+}$complex is localized on the metal and $\mathrm{C}^{\wedge} \mathrm{N}$ ligand, ${ }^{8}$ and therefore the energy of the HOMO can be altered by introducing electron-withdrawing or electron- donating substituents into the phenyl ring. This strategy has been successfully applied to tune orbital energies and emission properties of cyclometallated iridium(III) complexes in organic light-emitting diodes (OLEDs) and light-emitting electrochemical cells (LECs). ${ }^{9}$ An advantage of $\left[\mathrm{Ru}\left(\mathrm{N}^{\wedge} \mathrm{N}\right)_{2}\left(\mathrm{C}^{\wedge} \mathrm{N}\right)\right]^{+}$dyes in DSCs is the significant red-shift in their absorption spectra yielding improved photoresponse. The potential for enhanced photon-to-current conversion efficiencies has prompted active exploration of cyclometallated ruthenium(II) sensitizers for n-type DSCs. ${ }^{8,10,11}$ The effects of combining $\left[\mathrm{Ru}\left(\mathrm{N}^{\wedge} \mathrm{N}\right)_{2}\left(\mathrm{C}^{\wedge} \mathrm{N}\right)\right]^{+}$sensitizers with the cobalt-based redox shuttle $\left[\mathrm{Co}(\mathrm{dmbpy})_{3}\right]^{3+/ 2+}\left(\mathrm{dmbpy}=4,4^{\prime}\right.$-dimethyl-2,2'bipyridine) have also recently been reported. ${ }^{12}$

In $\left[\mathrm{Ru}\left(\mathrm{N}^{\wedge} \mathrm{N}\right)_{2}\left(\mathrm{C}^{\wedge} \mathrm{N}\right)\right]^{+}$dyes for n-type DSCs, the cyclometallating domain constitutes the ancillary ligand. However, the localization of the HOMO on the $\mathrm{C}^{\wedge} \mathrm{N}$ ligand and ruthenium means that $\left[\mathrm{Ru}\left(\mathrm{N}^{\wedge} \mathrm{N}\right)_{2}\left(\mathrm{C}^{\wedge} \mathrm{N}\right)\right]^{+}$complexes are of potential interest as sensitizers in either p-type or tandem DSCs. ${ }^{13}$ In a p-type DSC, excitation of the anchored dye is 
followed by rapid hole injection into a p-type semiconductor (e.g. NiO). This is the inverse of electron injection from dye to n-type semiconductor, leading to contrary frontier orbital requirements of dyes in $\mathrm{n}$ - and p-type DSCs. The viability of ruthenium(II) complexes containing cyclometallated ligands (both $\mathrm{C}^{\wedge} \mathrm{N}$ and $\mathrm{N}^{\wedge} \mathrm{C}^{\wedge} \mathrm{N}$ ) for $\mathrm{NiO}$ sensitization has been established, ${ }^{14,15}$ but investigations of these dyes remain limited.

We report here an investigation of two families of $\left[\operatorname{Ru}\left(\mathrm{N}^{\wedge} \mathrm{N}\right)_{2}\left(\mathrm{C}^{\wedge} \mathrm{N}\right)\right]^{n+}(n=0,1)$ complexes with various $\mathrm{C}^{\wedge} \mathrm{N}$ ligand-functionalization (Schemes 1 and 2) and the consequential effects on the absorption and electrochemical properties. Functionalization includes the introduction of different anchoring domains (Scheme 2). Both carboxylic and phosphonic acid substituents are ubiquitous among anchoring groups to attach sensitizers to $\mathrm{TiO}_{2}$ in n-type DSCs, and these anchors are also appropriate for adsorption of dyes on p-type NiO. ${ }^{16}$ In the current work, we focus on a phosphonate ester rather than phosphonic acid-functionalized $C^{\wedge} \mathrm{N}$ ligand (Scheme 2). Hydrolytic deprotection of the ruthenium-bound diethyl phosphonates does not always proceed to completion $^{17,18,19,20,21}$ and phosphonate esters have been successfully used to modify $\mathrm{TiO}_{2}$ surfaces, negating the need for this step. ${ }^{22,23}$

\section{Experimental}

\section{General}

Microwave reactions were carried out in a Biotage Initiator 8 reactor. ${ }^{1} \mathrm{H},{ }^{13} \mathrm{C}$ and ${ }^{31} \mathrm{P}$ NMR spectra were recorded on Bruker Avance III-250, III-400 or III-500 spectrometers at $295 \mathrm{~K}$. The chemical shifts were referenced with respect to residual solvent peaks $(\partial(T M S)=0 \mathrm{ppm})$. IR spectra were recorded on a Perkin Elmer FT-IR Spectrum Two spectrometer using solid samples. ESI mass spectra (solution samples in $\mathrm{MeCN}$ ) and high resolution ESI-MS were measured on Bruker Esquire $3000^{\text {plus }}$ and Bruker maXis 4G instruments, respectively; LC-ESI-MS and MALDI-TOF mass spectra employed a combination of Shimadzu (LC) and Bruker AmaZon X instruments or a Microflex Bruker Daltonics instrument, respectively. An Agilent 8453 spectrophotometer was used to record absorption spectra, and photoluminescence measurements were performed on a Shimadzu RF-5301 PC spectrofluorometer.

Electrochemical measurements were made with a CHI 900B instrument using a glassy carbon working electrode, platinumwire auxiliary electrode, and silver-wire pseudo-reference electrode. Redox potentials were determined by both cyclic and square wave voltammetry. HPLC grade, argon degassed MeCN solutions $\left(\approx 10^{-4} \mathrm{~mol} \mathrm{\textrm {dm } ^ { - 3 }}\right)$ of samples were used in the presence of $0.1 \mathrm{M}\left[{ }^{\mathrm{n}} \mathrm{Bu}_{4} \mathrm{~N}\right]\left[\mathrm{PF}_{6}\right]$ as supporting electrolyte; the scan rate was $0.1 \mathrm{~V} \mathrm{~s}^{-1}$ and ferrocene $\left(\mathrm{Fc}^{+} / \mathrm{Fc}\right)$ was the internal standard.

The compounds $\left[\mathrm{Ru}(\mathrm{bpy})_{2}(\mathrm{ppy})\right]\left[\mathrm{PF}_{6}\right],{ }^{1} \mathrm{H} \mathbf{1},{ }^{24} \mathrm{H} 2,{ }^{25} \mathrm{H} 3,{ }^{25}$ $\mathrm{H} 4,{ }^{26} \mathrm{H5},{ }^{27} \mathrm{H6},{ }^{27} \mathrm{H} 7,{ }^{28} \mathrm{H}_{2} 8^{8}$ were prepared by literature methods.

\section{2-Phenyl-4-(4-bromophenyl)pyridine-6-carboxylic acid}

$N$-Phenacylpyridinium chloride (3.79 g, $162 \mathrm{mmol}),(E)-4-(4-$ bromophenyl)-2-oxobut-3-enoic acid (4.14 g, $16.2 \mathrm{mmol})$ and $\mathrm{NH}_{4} \mathrm{OAc}(12.5 \mathrm{~g}, 162 \mathrm{mmol})$ were dissolved in $\mathrm{H}_{2} \mathrm{O}(120 \mathrm{~mL})$ and the solution heated at $100^{\circ} \mathrm{C}$ for $16 \mathrm{~h}$. The precipitate was separated by filtration and washed with water and acetone. After drying under vacuum, the product was obtained as an offwhite solid (2.09 g, $5.9 \mathrm{mmol}, 36 \%) .{ }^{1} \mathrm{H} \mathrm{NMR}(250 \mathrm{MHz}$, DMSO- $\left.d_{6}\right) \delta / \mathrm{ppm} 8.24-8.19\left(\mathrm{~m}, 2 \mathrm{H}, \mathrm{H}^{\mathrm{F} 2}\right), 8.16(\mathrm{~d}, J=1.7 \mathrm{~Hz}$, $\left.1 \mathrm{H}, \mathrm{H}^{\mathrm{E} 5 / \mathrm{E} 3}\right), 8.05\left(\mathrm{~d}, J=1.7 \mathrm{~Hz}, 1 \mathrm{H}, \mathrm{H}^{\mathrm{E} 3 / \mathrm{E} 5}\right), 7.91-7.85(\mathrm{~m}, 2 \mathrm{H}$, $\left.\mathrm{H}^{\mathrm{G} 2 / \mathrm{G} 3}\right), 7.77-7.65\left(\mathrm{~m}, 2 \mathrm{H}, \mathrm{H}^{\mathrm{G} 3 / \mathrm{G} 2}\right), 7.55-7.34\left(\mathrm{~m}, 3 \mathrm{H}, \mathrm{H}^{\mathrm{F} 3+\mathrm{F} 4}\right)$, $7.06\left(\mathrm{br}, 1 \mathrm{H}, \mathrm{H}^{\mathrm{COOH}}\right)$. The product was used in the next step without further characterization.

\section{2-Phenyl-4-(4-bromophenyl)pyridine}

2-Phenyl-4-(4-bromophenyl)pyridine-6-carboxylic acid (750 $\mathrm{mg}, 2.12 \mathrm{mmol})$ was suspended in ethane-1,2-diol $(200 \mathrm{~mL})$ and heated at $200^{\circ} \mathrm{C}$ for $24 \mathrm{~h}$. The resulting black solution was poured into water $(500 \mathrm{~mL})$ and extracted with $\mathrm{CH}_{2} \mathrm{Cl}_{2}(3 \times 20$ $\mathrm{mL})$. The organic layer was concentrated and purified by flash

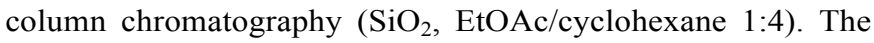
product was initially a brown oil that solidified on standing to give an off-white solid (440 mg, $1.42 \mathrm{mmol}, 67 \%) .{ }^{1} \mathrm{H}$ NMR $\left(500 \mathrm{MHz}, \mathrm{CDCl}_{3}\right) \delta / \mathrm{ppm}: 8.75\left(\mathrm{dd}, J=5.2,0.8 \mathrm{~Hz}, 1 \mathrm{H}, \mathrm{H}^{\mathrm{E} 6}\right)$, $8.07-8.02\left(\mathrm{~m}, 2 \mathrm{H}, \mathrm{H}^{\mathrm{F} 2}\right), 7.89\left(\mathrm{dd}, J=1.9,0.8 \mathrm{~Hz}, 1 \mathrm{H}, \mathrm{H}^{\mathrm{E} 3}\right)$, $7.64\left(\mathrm{~m}, 2 \mathrm{H}, \mathrm{H}^{\mathrm{G} 3}\right), 7.56\left(\mathrm{~m}, 2 \mathrm{H}, \mathrm{H}^{\mathrm{G} 2}\right), 7.50\left(\mathrm{~m}, 2 \mathrm{H}, \mathrm{H}^{\mathrm{F} 3}\right), 7.46$ $\left(\mathrm{m}, 1 \mathrm{H}, \mathrm{H}^{\mathrm{F} 4}\right), 7.43\left(\mathrm{dd}, J=5.2,1.8 \mathrm{~Hz}, 1 \mathrm{H}, \mathrm{H}^{\mathrm{E} 5}\right) .{ }^{13} \mathrm{C}\left\{{ }^{1} \mathrm{H}\right\}$

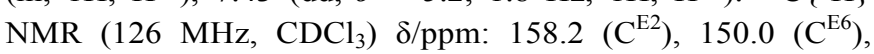
$148.6\left(\mathrm{C}^{\mathrm{E} 4}\right), 139.0\left(\mathrm{C}^{\mathrm{F} 1}\right), 137.4\left(\mathrm{C}^{\mathrm{G} 1}\right), 132.5\left(\mathrm{C}^{\mathrm{G} 3}\right), 129.5\left(\mathrm{C}^{\mathrm{F} 4}\right)$, $129.0\left(\mathrm{C}^{\mathrm{F} 3}\right), 128.8\left(\mathrm{C}^{\mathrm{G} 2}\right), 127.2\left(\mathrm{C}^{\mathrm{F} 2}\right), 123.8\left(\mathrm{C}^{\mathrm{G} 4}\right), 120.1\left(\mathrm{C}^{\mathrm{E} 5}\right)$, $118.7\left(\mathrm{C}^{\mathrm{E} 3}\right)$. LC-ESI-MS $\mathrm{m} / \mathrm{z}$ : $310.0[\mathrm{M}+\mathrm{H}]^{+}$(calc. 310.0 ). Found $\mathrm{C} 65.85, \mathrm{H} 4.19, \mathrm{~N} 4.48 ; \mathrm{C}_{17} \mathrm{H}_{12} \mathrm{BrN}$ requires $\mathrm{C} 65.83, \mathrm{H}$ $3.90, \mathrm{~N} 4.52$.

\section{Compound H9}

2-Phenyl-4-(4-bromophenyl)pyridine (400 mg, $1.29 \mathrm{mmol}$ ), $\mathrm{Pd}\left(\mathrm{PPh}_{3}\right)_{4}(149 \mathrm{mg}, 0.129 \mathrm{mmol})$ and $\mathrm{Cs}_{2} \mathrm{CO}_{3}(882 \mathrm{mg}, 2.71$ $\mathrm{mmol})$ were added to anhydrous THF $(10 \mathrm{~mL})$ in a microwave vial under argon. Diethyl phosphite (712 $\mathrm{mg}, 5.16 \mathrm{mmol})$ was added and the reaction mixture heated at $110^{\circ} \mathrm{C}$ for $90 \mathrm{~min}$ in a microwave reactor. The reaction mixture was filtered giving a yellow solution. Solvent was evaporated under reduced pressure, the brown residue dissolved in $\mathrm{CH}_{2} \mathrm{Cl}_{2}(10 \mathrm{~mL})$ and the mixture stirred with charcoal for $30 \mathrm{~min}$. Filtration through Celite prior to removal of the solvent gave a bright yellow oily residue. This was dissolved in acetone $(5 \mathrm{~mL})$ and filtered through a short silica plug eluting with acetone/cyclohexane 2:3. Flash chromatography $\left(\mathrm{SiO}_{2}\right.$, EtOAc) followed by preparative TLC $\left(\mathrm{SiO}_{2}\right.$, cyclohexane/acetone 2:3) yielded $\mathrm{H} 9$ as a white solid (268 mg, $0.729 \mathrm{mmol}, 57 \%)$. ${ }^{1} \mathrm{H}$ NMR (500 MHz, $\left.295 \mathrm{~K}, \mathrm{CD}_{3} \mathrm{CN}\right) \delta / \mathrm{ppm}: 8.73\left(\mathrm{dd}, J=5.1,0.9 \mathrm{~Hz}, 1 \mathrm{H}, \mathrm{H}^{\mathrm{E} 6}\right)$, $8.16-8.14\left(\mathrm{~m}, 2 \mathrm{H}, \mathrm{H}^{\mathrm{F} 2}\right), 8.12\left(\mathrm{dd}, J=1.7,0.8 \mathrm{~Hz}, 1 \mathrm{H}, \mathrm{H}^{\mathrm{E} 3}\right)$, 7.95-7.87 (m, 4H, $\left.\mathrm{H}^{\mathrm{G} 2+\mathrm{G} 3}\right), 7.60\left(\mathrm{dd}, J=5.1,1.7 \mathrm{~Hz}, 1 \mathrm{H}, \mathrm{H}^{\mathrm{E} 5}\right)$, 7.54-7.45 (m, 3H, $\left.\mathrm{H}^{\mathrm{F} 3+\mathrm{F} 4}\right)$, 4.15-4.04 (m, $\left.4 \mathrm{H}, \mathrm{H}^{\mathrm{PO}\left(\mathrm{OCH}_{2} \mathrm{Me}\right)}{ }_{2}\right)$, $1.30\left(\mathrm{td}, J=7.0,0.6 \mathrm{~Hz}, 6 \mathrm{H}, \mathrm{H}^{\mathrm{PO}\left(\mathrm{OCH}_{2} M e\right)}{ }_{2}\right) .{ }^{13} \mathrm{C}\left\{{ }^{1} \mathrm{H}\right\} \mathrm{NMR}(126$ 
$\left.\mathrm{MHz}, \mathrm{CD}_{3} \mathrm{CN}\right) \delta / \mathrm{ppm}: 158.5\left(\mathrm{C}^{\mathrm{E} 2}\right), 151.0\left(\mathrm{C}^{\mathrm{E} 6}\right), 149.1\left(\mathrm{C}^{\mathrm{E} 4}\right)$, $142.9\left(\mathrm{~d}, J=3.2 \mathrm{~Hz}, \mathrm{C}^{\mathrm{G} 1}\right), 139.8\left(\mathrm{C}^{\mathrm{F} 1}\right), 133.2(\mathrm{~d}, J=10 \mathrm{~Hz}$, $\left.\mathrm{C}^{\mathrm{G} 3}\right), 130.7\left(\mathrm{~d}, J=187.8 \mathrm{~Hz}, \mathrm{C}^{\mathrm{G} 4}\right), 130.3\left(\mathrm{C}^{\mathrm{F} 4}\right), 129.7\left(\mathrm{C}^{\mathrm{F} 3}\right)$, $128.4\left(\mathrm{~d}, J=14.9 \mathrm{~Hz}, \mathrm{C}^{\mathrm{G} 2}\right), 128.0\left(\mathrm{C}^{\mathrm{F} 2}\right), 121.5\left(\mathrm{C}^{\mathrm{E} 5}\right), 119.6$ $\left(\mathrm{C}^{\mathrm{E} 3}\right), 63.1\left(\mathrm{~d}, J=5.6 \mathrm{~Hz}, 2 \mathrm{C}, \mathrm{C}_{2}^{\mathrm{PO}\left(\mathrm{OCH}_{2} \mathrm{Me}\right)}{ }_{2}\right), 16.7(\mathrm{~d}, J=6.2$ $\left.\mathrm{Hz}, 2 \mathrm{C}, \mathrm{C}^{\mathrm{PO}\left(\mathrm{OCH}_{2} \mathrm{Me}\right)}{ }_{2}\right) .{ }^{31} \mathrm{P}\left\{{ }^{1} \mathrm{H}\right\} \mathrm{NMR}\left(162 \mathrm{MHz}, \mathrm{CD}_{3} \mathrm{CN}\right) \delta /$ ppm: 17.1. ESI MS m/z: $368.4[\mathrm{M}+\mathrm{H}]^{+}$(calc. 368.1), 390.4 $[\mathrm{M}+\mathrm{Na}]^{+}$(calc. 390.1). Found $\mathrm{C}$ 67.24, H 6.24, N 3.58; $\mathrm{C}_{21} \mathrm{H}_{22} \mathrm{NO}_{3} \mathrm{P} \cdot 0.5 \mathrm{H}_{2} \mathrm{O}$ requires $\mathrm{C} 67.01, \mathrm{H} 6.16, \mathrm{~N} 3.81$.

\section{$\left[\operatorname{Ru}(\text { bpy })_{2}\left({\text { ppy })]\left[P_{6}\right]}\right.\right.$}

The compound was prepared as previously reported. ${ }^{1}$ For comparative purposes, the NMR spectra were recorded and assigned as follows. ${ }^{1} \mathrm{H}$ NMR $\left(500 \mathrm{MHz}, \mathrm{CD}_{3} \mathrm{CN}\right) \delta / \mathrm{ppm}: 8.45$ $\left(\mathrm{dt}, J=8.3,1.1 \mathrm{~Hz}, 1 \mathrm{H}, \mathrm{H}^{\mathrm{A} 3}\right), 8.38(\mathrm{ddd}, J=8.2,1.4,0.8 \mathrm{~Hz}$, $\left.1 \mathrm{H}, \mathrm{H}^{\mathrm{B} 3}\right), 8.32\left(\mathrm{~m}, 1 \mathrm{H}, \mathrm{H}^{\mathrm{C} 3}\right), 8.30\left(\mathrm{~m}, 1 \mathrm{H}, \mathrm{H}^{\mathrm{D} 3}\right), 8.05(\mathrm{ddd}, J=$ $\left.5.7,1.6,0.8 \mathrm{~Hz}, 1 \mathrm{H}, \mathrm{H}^{\mathrm{B} 6}\right), 8.01\left(\mathrm{dt}, J=8.3,1.2 \mathrm{~Hz}, 1 \mathrm{H}, \mathrm{H}^{\mathrm{E} 3}\right)$, 7.97 (ddd, $\left.J=8.2,7.6,1.6 \mathrm{~Hz}, 1 \mathrm{H}, \mathrm{H}^{\mathrm{A} 4}\right), 7.85-7.76(\mathrm{~m}, 5 \mathrm{H}$, $\left.\mathrm{H}^{\mathrm{A} 6+\mathrm{F} 3+\mathrm{C} 4+\mathrm{B} 4+\mathrm{D} 6}\right), 7.77\left(\mathrm{ddd}, J=8.2,7.7,1.4 \mathrm{~Hz}, 1 \mathrm{H}, \mathrm{H}^{\mathrm{D} 4}\right), 7.72$ (ddd, $\left.J=5.7,1.5,0.8 \mathrm{~Hz}, 1 \mathrm{H}, \mathrm{H}^{\mathrm{C} 6}\right), 7.68(\mathrm{ddd}, J=8.2,7.4,1.6$ $\mathrm{Hz}, 1 \mathrm{H}, \mathrm{H}^{\mathrm{E} 4}$ ), 7.56 (ddd, $\left.J=5.7,1.6,0.8 \mathrm{~Hz}, 1 \mathrm{H}, \mathrm{H}^{\mathrm{E} 6}\right), 7.40$ (ddd, $\left.J=7.6,5.4,1.2 \mathrm{~Hz}, 1 \mathrm{H}, \mathrm{H}^{\mathrm{A} 5}\right), 7.22-7.18(\mathrm{~m}, 3 \mathrm{H}$, $\left.\mathrm{H}^{\mathrm{C} 5+\mathrm{D} 5+\mathrm{B} 5}\right), 6.92\left(\mathrm{ddd}, J=7.2,5.7,1.5 \mathrm{~Hz}, 1 \mathrm{H}, \mathrm{H}^{\mathrm{E} 5}\right), 6.89$ (ddd, $\left.J=7.4,7.3,1.3 \mathrm{~Hz}, 1 \mathrm{H}, \mathrm{H}^{\mathrm{F} 4}\right), 6.82(\mathrm{dt}, J=7.3,1.4 \mathrm{~Hz}, 1 \mathrm{H}$, $\left.\mathrm{H}^{\mathrm{F} 5}\right), 6.41\left(\mathrm{ddd}, J=7.4,1.3,0.5 \mathrm{~Hz}, 1 \mathrm{H}, \mathrm{H}^{\mathrm{F} 6}\right) .{ }^{13} \mathrm{C}\left\{{ }^{1} \mathrm{H}\right\} \mathrm{NMR}$ $\left(126 \mathrm{MHz}, \mathrm{CD}_{3} \mathrm{CN}\right) \delta / \mathrm{ppm}: 193.8\left(\mathrm{C}^{\mathrm{F} 1}\right), 168.3\left(\mathrm{C}^{\mathrm{E} 2}\right), 158.7$ $\left(\mathrm{C}^{\mathrm{B} 2}\right), 157.8\left(\mathrm{C}^{\mathrm{C} 2}\right), 157.6\left(\mathrm{C}^{\mathrm{D} 2}\right), 156.1\left(\mathrm{C}^{\mathrm{A} 2}\right), 155.0\left(\mathrm{C}^{\mathrm{B} 6}\right), 151.3$ $\left(\mathrm{C}^{\mathrm{E} 6}\right), 151.2\left(\mathrm{C}^{\mathrm{D} 6}\right), 151.0\left(\mathrm{C}^{\mathrm{C} 6}\right), 150.0\left(\mathrm{C}^{\mathrm{A} 6}\right), 146.6\left(\mathrm{C}^{\mathrm{F} 2}\right), 137.2$ $\left(\mathrm{C}^{\mathrm{A} 4}\right), 136.7\left(\mathrm{C}^{\mathrm{E} 4}\right), 136.2\left(\mathrm{C}^{\mathrm{F} 6}\right), 135.9\left(\mathrm{C}^{\mathrm{B} 4}\right), 134.7\left(\mathrm{C}^{\mathrm{C} 4}\right), 134.5$ $\left(\mathrm{C}^{\mathrm{D} 4}\right), 129.4\left(\mathrm{C}^{\mathrm{F} 5}\right), 128.0\left(\mathrm{C}^{\mathrm{A} 5}\right), 127.3\left(\mathrm{C}^{\mathrm{C} 5}\right), 127.2\left(\mathrm{C}^{\mathrm{B} 5}\right), 127.0$ $\left(\mathrm{C}^{\mathrm{D} 5}\right), 125.8\left(\mathrm{C}^{\mathrm{F} 3}\right), 124.4\left(\mathrm{C}^{\mathrm{B} 3}\right), 124.1\left(\mathrm{C}^{\mathrm{A} 3}\right), 124.0\left(\mathrm{C}^{\mathrm{D} 3}\right), 123.9$ $\left(\mathrm{C}^{\mathrm{C} 3}\right), 123.2\left(\mathrm{C}^{\mathrm{E} 5}\right), 121.8\left(\mathrm{C}^{\mathrm{F} 4}\right), 119.8\left(\mathrm{C}^{\mathrm{E} 3}\right)$.

\section{$\left[\mathrm{Ru}(\mathrm{bpy})_{2}(\mathbf{1})\right]\left[\mathrm{PF}_{6}\right]$}

cis-[Ru(bpy $\left.)_{2} \mathrm{Cl}_{2}\right](50.0 \mathrm{mg}, 0.103 \mathrm{mmol}), \mathrm{H} 1$ (49.0 mg, 0.283 $\mathrm{mmol}$ ) and $\mathrm{AgBF}_{4}(40.2 \mathrm{mg}, 0.206 \mathrm{mmol})$ were dissolved in $\mathrm{CH}_{2} \mathrm{Cl}_{2}(7.5 \mathrm{~mL})$ and the dark purple solution heated at reflux for $24 \mathrm{~h}$. The reaction mixture was filtered through Celite to remove precipitated $\mathrm{AgCl}$. The dark red-purple solution was concentrated under reduced pressure. $n$-Hexane $(50 \mathrm{~mL})$ was added to the dark purple oily residue and the mixture was left in a refrigerator overnight. The solution was decanted to separate a dark purple-red precipitate which was dried under reduced pressure. The solid was dissolved in $\mathrm{MeCN}(10 \mathrm{~mL})$ and an excess of aqueous $\mathrm{NH}_{4} \mathrm{PF}_{6}$ was added. The precipitate was collected by filtration, washed with $\mathrm{H}_{2} \mathrm{O}$ and $\mathrm{Et}_{2} \mathrm{O}$ and redissolved with $\mathrm{CH}_{2} \mathrm{Cl}_{2}$. The solution was concentrated under reduced pressure and purified by flash chromatography $\left(\mathrm{Al}_{2} \mathrm{O}_{3}\right.$, acetone/toluene $1: 1$ changing to $4: 1$ and pure acetone). $\left[\mathrm{Ru}(\mathrm{bpy})_{2}(\mathbf{1})\right]\left[\mathrm{PF}_{6}\right]$ was obtained as a dark purple solid (39 $\mathrm{mg}$, $0.053 \mathrm{mmol}, 52 \%) .{ }^{1} \mathrm{H}$ NMR (500 MHz, $\left.\mathrm{CD}_{3} \mathrm{CN}\right) \delta / \mathrm{ppm}: 8.45$ $\left(\mathrm{dt}, J=8.2,1.1 \mathrm{~Hz}, 1 \mathrm{H}, \mathrm{H}^{\mathrm{A} 3}\right), 8.38(\mathrm{dt}, J=8.2,1.2 \mathrm{~Hz}, 1 \mathrm{H}$, $\left.\mathrm{H}^{\mathrm{B} 3}\right), 8.33\left(\mathrm{~m}, 1 \mathrm{H}, \mathrm{H}^{\mathrm{C} 3}\right), 8.31\left(\mathrm{~m}, 1 \mathrm{H}, \mathrm{H}^{\mathrm{D} 3}\right), 8.04(\mathrm{ddd}, J=5.7$, 1.6, $\left.0.8 \mathrm{~Hz}, 1 \mathrm{H}, \mathrm{H}^{\mathrm{B} 6}\right), 7.98\left(\mathrm{~m}, 2 \mathrm{H}, \mathrm{H}^{\mathrm{A} 4+\mathrm{E} 3}\right), 7.88-7.79(\mathrm{~m}, 5 \mathrm{H}$, $\left.\mathrm{H}^{\mathrm{F} 3+\mathrm{C} 4+\mathrm{A} 6+\mathrm{B} 4+\mathrm{D} 4}\right), 7.78$ (ddd, $\left.J=5.8,1.5,0.8 \mathrm{~Hz}, 1 \mathrm{H}, \mathrm{H}^{\mathrm{D} 6}\right), 7.71$ (ddd, $\left.J=5.7,1.5,0.8 \mathrm{~Hz}, 1 \mathrm{H}, \mathrm{H}^{\mathrm{C} 6}\right), 7.68(\mathrm{ddd}, J=8.2,7.4,1.6$
$\left.\mathrm{Hz}, 1 \mathrm{H}, \mathrm{H}^{\mathrm{E} 4}\right), 7.54\left(\mathrm{ddd}, J=5.7,1.6,0.8 \mathrm{~Hz}, 1 \mathrm{H}, \mathrm{H}^{\mathrm{E} 6}\right), 7.41$ (ddd, $\left.J=7.6,5.4,1.2 \mathrm{~Hz}, 1 \mathrm{H}, \mathrm{H}^{\mathrm{A} 5}\right), 7.22\left(\mathrm{~m}, 3 \mathrm{H}, \mathrm{H}^{\mathrm{C} 5+\mathrm{D} 5+\mathrm{B} 5}\right)$, $6.92\left(\mathrm{ddd}, J=7.3,5.7,1.4 \mathrm{~Hz}, 1 \mathrm{H}, \mathrm{H}^{\mathrm{E} 5}\right), 6.60(\mathrm{ddd}, J=9.3,8.5$, $\left.2.7 \mathrm{~Hz}, 1 \mathrm{H}, \mathrm{H}^{\mathrm{F} 4}\right), 6.05\left(\mathrm{dd}, J=9.6,2.7 \mathrm{~Hz}, 1 \mathrm{H}, \mathrm{H}^{\mathrm{F} 6}\right) ;{ }^{13} \mathrm{C}\left\{{ }^{1} \mathrm{H}\right\}$ NMR (126 MHz, CD $\left.{ }_{3} \mathrm{CN}\right) \delta / p p m: 198.9\left(\mathrm{~d}, J=3.2 \mathrm{~Hz}, \mathrm{C}^{\mathrm{F} 1}\right)$, $167.3\left(\mathrm{C}^{\mathrm{E} 2}\right), 164.0\left(\mathrm{~d}, J=252 \mathrm{~Hz}, \mathrm{C}^{\mathrm{F} 5}\right), 158.6\left(\mathrm{C}^{\mathrm{B} 2}\right), 157.9$ $\left(\mathrm{C}^{\mathrm{C} 2}\right), 157.6\left(\mathrm{C}^{\mathrm{D} 2}\right), 156.1\left(\mathrm{C}^{\mathrm{A} 2}\right), 155.1\left(\mathrm{C}^{\mathrm{B} 6}\right), 151.3\left(\mathrm{C}^{\mathrm{E} 6}\right), 151.2$ $\left(\mathrm{C}^{\mathrm{D} 6}\right), 151.1\left(\mathrm{C}^{\mathrm{C} 6}\right), 150.2\left(\mathrm{C}^{\mathrm{A} 6}\right), 142.9\left(\mathrm{~d}, J=1.9 \mathrm{~Hz}, \mathrm{C}^{\mathrm{F} 2}\right)$, $137.5\left(\mathrm{C}^{\mathrm{A} 4}\right), 136.8\left(\mathrm{C}^{\mathrm{E} 4}\right), 136.1\left(\mathrm{C}^{\mathrm{B} 4}\right), 135.1\left(\mathrm{C}^{\mathrm{C} 4}\right), 135.0\left(\mathrm{C}^{\mathrm{D} 4}\right)$, $128.0\left(\mathrm{C}^{\mathrm{A} 5}\right), 127.5\left(\mathrm{C}^{\mathrm{C} 5}\right), 127.3\left(\mathrm{C}^{\mathrm{B} 5}\right), 127.2\left(\mathrm{C}^{\mathrm{D} 5}\right), 126.8(\mathrm{~d}, J=$ $\left.8.7 \mathrm{~Hz}, \mathrm{C}^{\mathrm{F} 3}\right), 124.4\left(\mathrm{C}^{\mathrm{B} 3}\right), 124.2\left(\mathrm{C}^{\mathrm{A} 3}\right), 124.1\left(2 \mathrm{C}, \mathrm{C}^{\mathrm{C} 3+\mathrm{D} 3}\right)$, $123.1\left(\mathrm{C}^{\mathrm{E} 5}\right), 121.2\left(\mathrm{~d}, J=14.4 \mathrm{~Hz} \mathrm{C}^{\mathrm{F} 6}\right), 119.7\left(\mathrm{C}^{\mathrm{E} 3}\right), 108.7(\mathrm{~d}, J$ $\left.=23.5 \mathrm{~Hz}, \mathrm{C}^{\mathrm{F} 4}\right) .{ }^{19} \mathrm{~F}\left\{{ }^{1} \mathrm{H}\right\}$ NMR $\left(377 \mathrm{MHz}, \mathrm{CD}_{3} \mathrm{CN}\right) \delta / \mathrm{ppm}$ :$72.9\left(\mathrm{~d}, J=706 \mathrm{~Hz}, 6 \mathrm{~F},\left[\mathrm{PF}_{6}\right]^{-}\right),-114.5\left(\mathrm{~s}, 1 \mathrm{~F}, \mathrm{~F}^{\mathrm{F} 5}\right)$. ESI-MS $m / z: 586.4\left[\mathrm{M}-\mathrm{PF}_{6}\right]^{+}$(calc. 586.1). UV-Vis $\left(\mathrm{MeCN}, 1 \times 10^{-5}\right.$ M) $\lambda / \mathrm{nm}\left(\varepsilon / \mathrm{dm}^{3} \mathrm{~mol}^{-1} \mathrm{~cm}^{-1}\right): 248$ (33900), 296 (61300), 368 (11600), 399 (11200), 485 (9000), 538 (10200). Emission $\left(\mathrm{MeCN}, \mathrm{c}=5 \times 10^{-5} \mathrm{M}, \lambda_{\text {exc }}=560 \mathrm{~nm}\right): \lambda_{\max }{ }^{\mathrm{em}}=776 \mathrm{~nm}$. Found $\mathrm{C} 49.77, \mathrm{H} 3.53, \mathrm{~N} 9.36 ; \mathrm{C}_{31} \mathrm{H}_{23} \mathrm{~F}_{7} \mathrm{~N}_{5} \mathrm{PRu} \cdot \mathrm{H}_{2} \mathrm{O}$ requires C 49.74, H 3.37, N 9.36.

\section{$\left[\mathbf{R u}(\mathrm{bpy})_{2}(2)\right]\left[\mathrm{PF}_{6}\right]$}

cis-[Ru(bpy) $\left.)_{2} \mathrm{Cl}_{2}\right](120 \mathrm{mg}, 0.248 \mathrm{mmol}), \mathrm{H} 2(92.2 \mathrm{mg}, 0.09$ $\mathrm{mL}, 0.545 \mathrm{mmol})$ and $\mathrm{AgPF}_{6}(138 \mathrm{mg}, 0.545 \mathrm{mmol})$ were dissolved in $\mathrm{CH}_{2} \mathrm{Cl}_{2} / \mathrm{MeOH}(3: 1,16 \mathrm{~mL})$ and the dark purple solution heated at reflux for $2 \mathrm{~h}$. The mixture was filtered through Celite and the filtrate concentrated under reduced pressure and subjected to flash chromatography $\left(\mathrm{Al}_{2} \mathrm{O}_{3}\right.$, acetone/pentane 1:2 changing to pure acetone). Fractions were combined and solvent volume reduced; $n$-hexane was then added to precipitate a dark purple solid. $\mathrm{CH}_{2} \mathrm{Cl}_{2}(20 \mathrm{~mL})$ was added and the solution was layered with $\mathrm{Et}_{2} \mathrm{O}(100 \mathrm{~mL})$. $\left[\mathrm{Ru}(\mathrm{bpy})_{2}(\mathbf{2})\right]\left[\mathrm{PF}_{6}\right]$ precipitated as a dark purple crystalline solid after a few days (108 mg, $0.149 \mathrm{mmol}, 60 \%) .{ }^{1} \mathrm{H}$ NMR (500 MHz, $\left.\mathrm{CD}_{3} \mathrm{CN}\right) \delta / \mathrm{ppm}: 8.44\left(\mathrm{dt}, J=8.2,1.1 \mathrm{~Hz}, 1 \mathrm{H}, \mathrm{H}^{\mathrm{A} 3}\right)$, $8.37\left(\mathrm{ddd}, J=8.2,1.3,0.7 \mathrm{~Hz}, 1 \mathrm{H}, \mathrm{H}^{\mathrm{B} 3}\right), 8.32\left(\mathrm{~m}, 1 \mathrm{H}, \mathrm{H}^{\mathrm{C} 3}\right)$, $8.30\left(\mathrm{~m}, 1 \mathrm{H}, \mathrm{H}^{\mathrm{D} 3}\right), 8.07\left(\mathrm{ddd}, J=5.7,1.6,0.8 \mathrm{~Hz}, 1 \mathrm{H}, \mathrm{H}^{\mathrm{B} 6}\right)$, 7.97 (ddd, $\left.J=8.2,7.6,1.6 \mathrm{~Hz}, 1 \mathrm{H}, \mathrm{H}^{\mathrm{A} 4}\right), 7.95(\mathrm{dt}, J=8.3,1.2$ $\left.\mathrm{Hz}, 1 \mathrm{H}, \mathrm{H}^{\mathrm{E} 3}\right), 7.83-7.76\left(\mathrm{~m}, 5 \mathrm{H}, \mathrm{H}^{\mathrm{A} 6+\mathrm{C} 4+\mathrm{B} 4+\mathrm{D} 6+\mathrm{D} 4}\right), 7.73(\mathrm{~d}, J=$ $\left.7.8 \mathrm{~Hz}, 1 \mathrm{H}, \mathrm{H}^{\mathrm{F} 3}\right), 7.71\left(\mathrm{ddd}, J=5.7,1.5,0.8 \mathrm{~Hz}, 1 \mathrm{H}, \mathrm{H}^{\mathrm{C} 6}\right), 7.65$ (ddd, $\left.J=8.2,7.4,1.6 \mathrm{~Hz}, 1 \mathrm{H}, \mathrm{H}^{\mathrm{E} 4}\right), 7.51(\mathrm{ddd}, J=5.7,1.6,0.8$ $\mathrm{Hz}, 1 \mathrm{H}, \mathrm{H}^{\mathrm{E} 6}$ ), 7.39 (ddd, $\left.J=7.6,5.4,1.2 \mathrm{~Hz}, 1 \mathrm{H}, \mathrm{H}^{\mathrm{A} 5}\right), 7.22-$ $7.18\left(\mathrm{~m}, 3 \mathrm{H}, \mathrm{H}^{\mathrm{C} 5+\mathrm{D} 5+\mathrm{B} 5}\right), 6.87(\mathrm{ddd}, J=7.3,5.7,1.4 \mathrm{~Hz}, 1 \mathrm{H}$, $\left.\mathrm{H}^{\mathrm{E} 5}\right), 6.71\left(\mathrm{ddd}, J=8.0,1.9,0.7 \mathrm{~Hz}, 1 \mathrm{H}, \mathrm{H}^{\mathrm{F} 4}\right), 6.24(\mathrm{dt}, J=1.9$, $\left.0.6 \mathrm{~Hz}, 1 \mathrm{H}, \mathrm{H}^{\mathrm{F} 6}\right), 2.03\left(\mathrm{~s}, 3 \mathrm{H}, \mathrm{H}^{\mathrm{Me}}\right) .{ }^{13} \mathrm{C}\left\{{ }^{1} \mathrm{H}\right\} \mathrm{NMR}(126 \mathrm{MHz}$, $\left.\mathrm{CD}_{3} \mathrm{CN}\right) \delta /$ ppm: $193.8\left(\mathrm{C}^{\mathrm{F} 1}\right), 168.3\left(\mathrm{C}^{\mathrm{E} 2}\right), 158.7\left(\mathrm{C}^{\mathrm{B} 2}\right), 157.8$ $\left(\mathrm{C}^{\mathrm{C} 2}\right), 157.7\left(\mathrm{C}^{\mathrm{D} 2}\right), 156.1\left(\mathrm{C}^{\mathrm{A} 2}\right), 155.0\left(\mathrm{C}^{\mathrm{B} 6}\right), 151.2\left(\mathrm{C}^{\mathrm{E} 6}\right), 151.1$ $\left(\mathrm{C}^{\mathrm{D} 6}\right), 150.9\left(\mathrm{C}^{\mathrm{C} 6}\right), 150.0\left(\mathrm{C}^{\mathrm{A} 6}\right), 143.8\left(\mathrm{C}^{\mathrm{F} 2}\right), 139.1\left(\mathrm{C}^{\mathrm{F} 5}\right), 137.2$ $\left(\mathrm{C}^{\mathrm{A} 4}\right), 136.9\left(\mathrm{C}^{\mathrm{F} 6}\right), 136.6\left(\mathrm{C}^{\mathrm{E} 4}\right), 135.8\left(\mathrm{C}^{\mathrm{B} 4}\right), 134.6\left(\mathrm{C}^{\mathrm{C} 4}\right), 134.4$ $\left(\mathrm{C}^{\mathrm{D} 4}\right), 128.0\left(\mathrm{C}^{\mathrm{A} 5}\right), 127.3\left(\mathrm{C}^{\mathrm{C} 5}\right), 127.1\left(\mathrm{C}^{\mathrm{B} 5}\right), 127.0\left(\mathrm{C}^{\mathrm{D} 5}\right), 125.0$ $\left(\mathrm{C}^{\mathrm{F} 3}\right), 124.4\left(\mathrm{C}^{\mathrm{B} 3}\right), 124.1\left(\mathrm{C}^{\mathrm{A} 3}\right), 123.93\left(\mathrm{C}^{\mathrm{D} 3}\right), 123.88\left(\mathrm{C}^{\mathrm{C} 3}\right)$, $123.0\left(\mathrm{C}^{\mathrm{F} 4}\right), 122.7\left(\mathrm{C}^{\mathrm{E} 5}\right), 119.4\left(\mathrm{C}^{\mathrm{E} 3}\right), 21.8\left(\mathrm{C}^{\mathrm{Me}}\right)$. ESI MS $m / z$ : $582.4\left[\mathrm{M}-\mathrm{PF}_{6}\right]^{+}$(calc. 582.1). UV-Vis $\left(\mathrm{MeCN}, 1 \times 10^{-5} \mathrm{M}\right)$

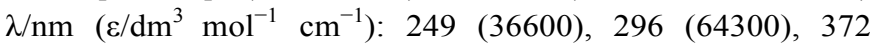
(11900), 405 (11300), 490 (8500), 548 (9500). Emission $\left(\mathrm{MeCN}, 5 \times 10^{-5} \mathrm{M}, \lambda_{\mathrm{exc}}=575 \mathrm{~nm}\right): \lambda_{\max }^{\mathrm{em}}=805 \mathrm{~nm}$. Found $\mathrm{C}$ 
52.62, $\mathrm{H}$ 3.95, N 9.66; $\mathrm{C}_{32} \mathrm{H}_{26} \mathrm{~F}_{6} \mathrm{~N}_{5} \mathrm{PRu}$ requires $\mathrm{C} 52.90, \mathrm{H}$ $3.61, \mathrm{~N} 9.64$.

\section{$\left[\mathrm{Ru}(\mathrm{bpy})_{2}(\mathbf{3})\right]\left[\mathrm{PF}_{6}\right]$}

cis-[Ru(bpy $\left.)_{2} \mathrm{Cl}_{2}\right](150 \mathrm{mg}, 0.310 \mathrm{mmol}), \mathrm{H} 3$ (126 mg, 0.681 $\mathrm{mmol})$ and $\mathrm{AgPF}_{6}(172 \mathrm{mg}, 0.681 \mathrm{mmol})$ were dissolved in $\mathrm{CH}_{2} \mathrm{Cl}_{2} / \mathrm{MeOH}(3: 1,16 \mathrm{~mL})$ and the dark purple solution heated at reflux for $2 \mathrm{~h}$. The mixture was filtered through Celite and the filtrate concentrated under reduced pressure and subjected to flash chromatography (basic $\mathrm{Al}_{2} \mathrm{O}_{3}$, EtOAc/cyclohexane 1:4 then pure $\mathrm{CH}_{2} \mathrm{Cl}_{2}$ ). The fractions were concentrated, and $n$-hexane was added to precipitate dark purple $\left[\mathrm{Ru}(\mathrm{bpy})_{2}(\mathbf{3})\right]\left[\mathrm{PF}_{6}\right](99 \mathrm{mg}, 0.133 \mathrm{mmol}, 43 \%) .{ }^{1} \mathrm{H} \mathrm{NMR}$ $\left(500 \mathrm{MHz}, \mathrm{CD}_{3} \mathrm{CN}\right) \delta / \mathrm{ppm}: 8.45\left(\mathrm{dt}, J=8.2,1.1 \mathrm{~Hz}, 1 \mathrm{H}, \mathrm{H}^{\mathrm{A} 3}\right)$, 8.37 (ddd, $\left.J=8.2,1.4,0.8 \mathrm{~Hz}, 1 \mathrm{H}, \mathrm{H}^{\mathrm{B} 3}\right), 8.31\left(\mathrm{~m}, 1 \mathrm{H}, \mathrm{H}^{\mathrm{C} 3}\right)$, $8.30\left(\mathrm{~m}, 1 \mathrm{H}, \mathrm{H}^{\mathrm{D} 3}\right), 8.09\left(\mathrm{ddd}, J=5.7,1.6,0.8 \mathrm{~Hz}, 1 \mathrm{H}, \mathrm{H}^{\mathrm{B} 6}\right)$, $7.97\left(\mathrm{ddd}, J=8.2,7.5,1.6 \mathrm{~Hz}, 1 \mathrm{H}, \mathrm{H}^{\mathrm{A} 4}\right), 7.86\left(\mathrm{~m}, 2 \mathrm{H}, \mathrm{H}^{\mathrm{E} 3+\mathrm{A} 6}\right)$, 7.83-7.76 (m, 5H, $\left.\mathrm{H}^{\mathrm{D} 6+\mathrm{B} 4+\mathrm{D} 4+\mathrm{F} 3+\mathrm{C} 4}\right), 7.73(\mathrm{ddd}, J=5.7,1.5,0.8$ $\left.\mathrm{Hz}, 1 \mathrm{H}, \mathrm{H}^{\mathrm{C} 6}\right), 7.62\left(\mathrm{ddd}, J=8.2,7.4,1.6 \mathrm{~Hz}, 1 \mathrm{H}, \mathrm{H}^{\mathrm{E} 4}\right), 7.48$ $\left(\mathrm{ddd}, J=5.7,1.6,0.8 \mathrm{~Hz}, 1 \mathrm{H}, \mathrm{H}^{\mathrm{E} 6}\right), 7.40$ (ddd, $J=7.6,5.4,1.2$ $\left.\mathrm{Hz}, 1 \mathrm{H}, \mathrm{H}^{\mathrm{A} 5}\right), 7.21\left(\mathrm{~m}, 3 \mathrm{H}, \mathrm{H}^{\mathrm{C} 5+\mathrm{D} 5+\mathrm{B} 5}\right), 6.83(\mathrm{ddd}, J=7.2,5.7$, $\left.1.4 \mathrm{~Hz}, 1 \mathrm{H}, \mathrm{H}^{\mathrm{E} 5}\right), 6.46\left(\mathrm{dd}, J=8.5,2.6 \mathrm{~Hz}, 1 \mathrm{H}, \mathrm{H}^{\mathrm{F} 4}\right), 5.84(\mathrm{~d}, J$ $\left.=2.6 \mathrm{~Hz}, 1 \mathrm{H}, \mathrm{H}^{\mathrm{F} 6}\right), 3.52\left(\mathrm{~s}, 3 \mathrm{H}, \mathrm{H}^{\mathrm{OMe}}\right) .{ }^{13} \mathrm{C}\left\{{ }^{1} \mathrm{H}\right\} \mathrm{NMR}(126$ $\left.\mathrm{MHz}, \mathrm{CD}_{3} \mathrm{CN}\right) \delta / \mathrm{ppm}: 196.4\left(\mathrm{C}^{\mathrm{F} 1}\right), 168.0\left(\mathrm{C}^{\mathrm{E} 2}\right), 160.8\left(\mathrm{C}^{\mathrm{F} 5}\right)$, $158.7\left(\mathrm{C}^{\mathrm{B} 2}\right), 158.0\left(\mathrm{C}^{\mathrm{C} 2}\right), 157.7\left(\mathrm{C}^{\mathrm{D} 2}\right), 156.2\left(\mathrm{C}^{\mathrm{A} 2}\right), 155.0\left(\mathrm{C}^{\mathrm{B} 6}\right)$, $151.2\left(\mathrm{C}^{\mathrm{D} 6}\right), 151.1\left(\mathrm{C}^{\mathrm{C} 6}\right), 151.0\left(\mathrm{C}^{\mathrm{E} 6}\right), 150.2\left(\mathrm{C}^{\mathrm{A} 6}\right), 139.4\left(\mathrm{C}^{\mathrm{F} 2}\right)$, $137.3\left(\mathrm{C}^{\mathrm{A} 4}\right), 136.6\left(\mathrm{C}^{\mathrm{E} 4}\right), 135.9\left(\mathrm{C}^{\mathrm{B} 4}\right), 134.7\left(\mathrm{C}^{\mathrm{C} 4}\right), 134.6\left(\mathrm{C}^{\mathrm{D} 4}\right)$, $128.0\left(\mathrm{C}^{\mathrm{A} 5}\right), 127.3\left(\mathrm{C}^{\mathrm{C} 5}\right), 127.2\left(\mathrm{C}^{\mathrm{B} 5}\right), 127.0\left(\mathrm{C}^{\mathrm{D} 5}\right), 126.5\left(\mathrm{C}^{\mathrm{F} 3}\right)$, $124.4\left(\mathrm{C}^{\mathrm{B} 3}\right), 124.2\left(\mathrm{C}^{\mathrm{A} 3}\right), 124.0\left(\mathrm{C}^{\mathrm{D} 3}\right), 123.9\left(\mathrm{C}^{\mathrm{C} 3}\right), 122.1\left(\mathrm{C}^{\mathrm{E} 5}\right)$, $120.5\left(\mathrm{C}^{\mathrm{F} 6}\right), 119.0\left(\mathrm{C}^{\mathrm{E} 3}\right), 107.7\left(\mathrm{C}^{\mathrm{F} 4}\right), 55.1\left(\mathrm{C}^{\mathrm{OMe}}\right)$. ESI-MS $m / z$ : $598.3\left[\mathrm{M}-\mathrm{PF}_{6}\right]^{+}$(calc. 598.1). UV-Vis $\left(\mathrm{MeCN}, 1 \times 10^{-5} \mathrm{M}\right)$ $\lambda / \mathrm{nm}\left(\varepsilon / \mathrm{dm}^{3} \mathrm{~mol}^{-1} \mathrm{~cm}^{-1}\right): 252$ (37100), 296 (70800), 372 (11500), 398 (10900), 486 (8100), 547 (9400). Emission $\left(\mathrm{MeCN}, 5 \times 10^{-5} \mathrm{M}, \lambda_{\text {exc }}=570 \mathrm{~nm}\right): \lambda_{\max }{ }^{\mathrm{em}}=800 \mathrm{~nm}$. Found C 51.92, $\mathrm{H} 3.82, \mathrm{~N} 9.37 ; \mathrm{C}_{32} \mathrm{H}_{26} \mathrm{~F}_{6} \mathrm{~N}_{5} \mathrm{OPRu}$ requires $\mathrm{C} 51.76, \mathrm{H}$ $3.53, \mathrm{~N} 9.43$.

\section{$\left[\mathrm{Ru}(\mathrm{bpy})_{2}(4)\right]\left[\mathrm{PF}_{6}\right]$}

cis-[Ru(bpy) $\left.{ }_{2} \mathrm{Cl}_{2}\right](120 \mathrm{mg}, 0.247 \mathrm{mmol}), \mathrm{H} 4$ (116 mg, 0.543 mmol) and $\operatorname{AgPF}_{6}(137 \mathrm{mg}, 0.543 \mathrm{mmol})$ were dissolved in $\mathrm{CH}_{2} \mathrm{Cl}_{2} / \mathrm{MeOH}(3: 1,16 \mathrm{~mL})$ and the solution heated at reflux for $2 \mathrm{~h}$. The mixture was filtered through Celite, the volume of the filtrate reduced, and flash chromatography $\left(\mathrm{Al}_{2} \mathrm{O}_{3}\right.$, acetone/pentane 1:2 changing to acetone) used for purifiaction. The fractions were concentrated, and $n$-hexane was added to precipitate dark purple $\left[\mathrm{Ru}(\mathrm{bpy})_{2}(4)\right]\left[\mathrm{PF}_{6}\right](95 \mathrm{mg}, 0.123 \mathrm{mmol}$, $50 \%) .{ }^{1} \mathrm{H}$ NMR $\left(500 \mathrm{MHz}, \mathrm{CD}_{3} \mathrm{CN}\right) \delta / \mathrm{ppm}: 8.46(\mathrm{dt}, J=8.3$, $\left.1.1 \mathrm{~Hz}, 1 \mathrm{H}, \mathrm{H}^{\mathrm{A} 3}\right), 8.38\left(\mathrm{ddd}, J=8.2,1.3,0.7 \mathrm{~Hz}, 1 \mathrm{H}, \mathrm{H}^{\mathrm{B} 3}\right), 8.34$ (ddd, $J=8.2,1.4,0.8 \mathrm{~Hz}, 1 \mathrm{H}, \mathrm{H}^{\mathrm{C} 3}$ ), 8.31 (ddd, $J=8.2,1.4,0.8$ $\left.\mathrm{Hz}, 1 \mathrm{H}, \mathrm{H}^{\mathrm{D} 3}\right), 8.10\left(\mathrm{dt}, J=8.2,1.3 \mathrm{~Hz}, 1 \mathrm{H}, \mathrm{H}^{\mathrm{E} 3}\right), 7.99(\mathrm{~m}, 2 \mathrm{H}$, $\left.\mathrm{H}^{\mathrm{B} 6+\mathrm{A} 4}\right), 7.92\left(\mathrm{~d}, J=8.0 \mathrm{~Hz}, 1 \mathrm{H}, \mathrm{H}^{\mathrm{F} 3}\right), 7.85\left(\mathrm{~m}, 2 \mathrm{H}, \mathrm{H}^{\mathrm{C} 4+\mathrm{A} 6}\right)$, 7.83-7.72 (m, 5H, $\left.\mathrm{H}^{\mathrm{B} 4+\mathrm{D} 4+\mathrm{D} 6+\mathrm{E} 4+\mathrm{C} 6}\right), 7.63(\mathrm{ddd}, J=5.7,1.6,0.8$ $\left.\mathrm{Hz}, 1 \mathrm{H}, \mathrm{H}^{\mathrm{E} 6}\right), 7.49\left(\mathrm{dd}, J=8.1,1.8 \mathrm{~Hz}, 1 \mathrm{H}, \mathrm{H}^{\mathrm{F} 4}\right), 7.41(\mathrm{ddd}, J=$ 7.6, 5.4, 1.2 Hz, $1 \mathrm{H}, \mathrm{H}^{\mathrm{A} 5}$ ), 7.25 (ddd, $J=7.6,5.7,1.4 \mathrm{~Hz}, 1 \mathrm{H}$, $\left.\mathrm{H}^{\mathrm{C} 5}\right), 7.20\left(\mathrm{~m}, 2 \mathrm{H}, \mathrm{H}^{\mathrm{D} 5+\mathrm{B} 5}\right), 7.08\left(\mathrm{dd}, J=1.8,0.5 \mathrm{~Hz}, 1 \mathrm{H}, \mathrm{H}^{\mathrm{F} 6}\right)$, $7.00\left(\mathrm{ddd}, J=7.2,5.6,1.4 \mathrm{~Hz}, 1 \mathrm{H}, \mathrm{H}^{\mathrm{E} 5}\right), 3.69\left(\mathrm{~s}, 3 \mathrm{H}, \mathrm{H}_{2}^{\mathrm{CO}}{ }_{2}^{M e}\right)$.
${ }^{13} \mathrm{C}\left\{{ }^{1} \mathrm{H}\right\}$ NMR $\left(126 \mathrm{MHz}, \mathrm{CD}_{3} \mathrm{CN}\right) \delta / \mathrm{ppm}: 193.7\left(\mathrm{C}^{\mathrm{F} 1}\right), 168.5$ $\left(\mathrm{C}^{\mathrm{CO}}{ }_{2}^{\mathrm{Me}}\right), 167.2\left(\mathrm{C}^{\mathrm{E} 2}\right), 158.6\left(\mathrm{C}^{\mathrm{B} 2}\right), 157.8\left(\mathrm{C}^{\mathrm{C} 2}\right), 157.6\left(\mathrm{C}^{\mathrm{D} 2}\right)$, $156.1\left(\mathrm{C}^{\mathrm{A} 2}\right), 155.2\left(\mathrm{C}^{\mathrm{B} 6}\right), 151.7\left(\mathrm{C}^{\mathrm{E} 6}\right), 151.5\left(\mathrm{C}^{\mathrm{F} 2}\right), 151.3\left(\mathrm{C}^{\mathrm{D} 6}\right)$, $151.1\left(\mathrm{C}^{\mathrm{C} 6}\right), 150.1\left(\mathrm{C}^{\mathrm{A} 6}\right), 137.5\left(\mathrm{C}^{\mathrm{A} 4}\right), 136.9\left(\mathrm{C}^{\mathrm{E} 4}\right), 136.7\left(\mathrm{C}^{\mathrm{F} 6}\right)$, $136.1\left(\mathrm{C}^{\mathrm{B} 4}\right), 135.1\left(\mathrm{C}^{\mathrm{C} 4}\right), 134.9\left(\mathrm{C}^{\mathrm{D} 4}\right), 129.8\left(\mathrm{C}^{\mathrm{F} 5}\right), 128.0\left(\mathrm{C}^{\mathrm{A} 5}\right)$, $127.5\left(\mathrm{C}^{\mathrm{C} 5}\right), 127.4\left(\mathrm{C}^{\mathrm{B} 5}\right), 127.2\left(\mathrm{C}^{\mathrm{D} 5}\right), 124.6\left(\mathrm{C}^{\mathrm{F} 3}\right), 124.5\left(\mathrm{C}^{\mathrm{B} 3}\right)$, $124.3\left(\mathrm{C}^{\mathrm{E} 5}\right), 124.2\left(\mathrm{C}^{\mathrm{A} 3}\right), 124.1\left(\mathrm{C}^{\mathrm{D} 3}\right), 124.0\left(\mathrm{C}^{\mathrm{C} 3}\right), 122.9\left(\mathrm{C}^{\mathrm{F} 4}\right)$, $121.0\left(\mathrm{C}^{\mathrm{E} 3}\right), 52.3\left(\mathrm{C}_{2}^{\mathrm{CO}}{ }^{\mathrm{Me}}\right)$. ESI-MS $m / z: 626.4\left[\mathrm{M}-\mathrm{PF}_{6}\right]^{+}$(calc. 626.1). UV-Vis $\left(\mathrm{MeCN}, 1 \times 10^{-5} \mathrm{M}\right) \lambda / \mathrm{nm}\left(\varepsilon / \mathrm{dm}^{3} \mathrm{~mol}^{-1} \mathrm{~cm}^{-1}\right)$ : 250 (31600), 296 (70900), 360 (10900), 395 (7800), 485 (9400), 538 (10000). Emission (MeCN, $5 \times 10^{-5} \mathrm{M}, \lambda_{\mathrm{exc}}=565$ $\mathrm{nm}): \lambda_{\max }{ }^{\mathrm{em}}=781 \mathrm{~nm}$. Found $\mathrm{C} 50.93, \mathrm{H} 3.57, \mathrm{~N}$ 9.33; $\mathrm{C}_{33} \mathrm{H}_{26} \mathrm{~F}_{6} \mathrm{~N}_{5} \mathrm{O}_{2} \mathrm{PRu}$ requires $\mathrm{C} 51.43, \mathrm{H} 3.40, \mathrm{~N} 9.09$.

\section{$\left[\mathrm{Ru}(\mathrm{bpy})_{2}(\mathbf{5})\right]\left[\mathrm{PF}_{6}\right]$}

cis-[Ru(bpy) $\left.{ }_{2} \mathrm{Cl}_{2}\right](152 \mathrm{mg}, 0.314 \mathrm{mmol}), \mathrm{H} 5$ (168 mg, 0.691 $\mathrm{mmol})$ and $\mathrm{AgPF}_{6}(175 \mathrm{mg}, 0.691 \mathrm{mmol})$ were dissolved in $\mathrm{CH}_{2} \mathrm{Cl}_{2} / \mathrm{MeOH}(3: 1,20 \mathrm{~mL})$ and the solution heated at reflux for $2 \mathrm{~h}$. The reaction mixture was filtered through Celite, and the solution concentrated under reduced pressure and purified by flash chromatography $\left(\mathrm{Al}_{2} \mathrm{O}_{3}\right.$, EtOAc/pentane $1: 4$ changing to acetone). The fractions were concentrated under reduced pressure and $\left[\mathrm{Ru}(\mathrm{bpy})_{2}(\mathbf{5})\right]\left[\mathrm{PF}_{6}\right]$ was precipitated by addition of $n$-pentane. $\left[\mathrm{Ru}(\mathrm{bpy})_{2}(\mathbf{5})\right]\left[\mathrm{PF}_{6}\right]$ was isolated as a dark purple solid (67 mg, $0.083 \mathrm{mmol}, 26 \%) .{ }^{1} \mathrm{H} \mathrm{NMR}\left(500 \mathrm{MHz}, \mathrm{CD}_{3} \mathrm{CN}\right)$ $\delta /$ ppm: $8.46\left(\mathrm{dt}, J=8.2,1.1 \mathrm{~Hz}, 1 \mathrm{H}, \mathrm{H}^{\mathrm{A} 3}\right), 8.39(\mathrm{dt}, J=8.1,1.2$ $\left.\mathrm{Hz}, 1 \mathrm{H}, \mathrm{H}^{\mathrm{B} 3}\right), 8.32\left(\mathrm{dt}, J=8.2,1.1 \mathrm{~Hz}, 1 \mathrm{H}, \mathrm{H}^{\mathrm{C} 3}\right), 8.28(\mathrm{dt}, J=$ $\left.8.2,1.1 \mathrm{~Hz}, 1 \mathrm{H}, \mathrm{H}^{\mathrm{D} 3}\right), 8.05\left(\mathrm{ddd}, J=5.7,1.6,0.7 \mathrm{~Hz}, 1 \mathrm{H}, \mathrm{H}^{\mathrm{B} 6}\right)$, $8.02\left(\mathrm{dt}, J=8.2,1.1 \mathrm{~Hz}, 1 \mathrm{H}, \mathrm{H}^{\mathrm{E} 3}\right), 7.99(\mathrm{ddd}, J=8.2,7.6,1.6$ $\left.\mathrm{Hz}, 1 \mathrm{H}, \mathrm{H}^{\mathrm{A} 4}\right), 7.91$ (ddd, $J=5.4,1.6,0.8 \mathrm{~Hz}, 1 \mathrm{H}, \mathrm{H}^{\mathrm{A} 6}$ ), 7.84$7.75\left(\mathrm{~m}, 5 \mathrm{H}, \mathrm{H}^{\mathrm{C} 4+\mathrm{B} 4+\mathrm{D} 6+\mathrm{F} 3+\mathrm{D} 4}\right), 7.70\left(\mathrm{~m}, 2 \mathrm{H}, \mathrm{H}^{\mathrm{C} 6+\mathrm{E} 4}\right), 7.58(\mathrm{ddd}$, $\left.J=5.6,1.6,0.8 \mathrm{~Hz}, 1 \mathrm{H}, \mathrm{H}^{\mathrm{E} 6}\right), 7.42(\mathrm{ddd}, J=7.6,5.4,1.2 \mathrm{~Hz}$, $\left.1 \mathrm{H}, \mathrm{H}^{\mathrm{A} 5}\right), 7.24-7.18\left(\mathrm{~m}, 3 \mathrm{H}, \mathrm{H}^{\mathrm{C} 5+\mathrm{D} 5+\mathrm{B} 5}\right), 7.00(\mathrm{dd}, J=8.0,1.8$ $\left.\mathrm{Hz}, 1 \mathrm{H}, \mathrm{H}^{\mathrm{F} 4}\right), 6.95\left(\mathrm{ddd}, J=7.2,5.7,1.4 \mathrm{~Hz}, 1 \mathrm{H}, \mathrm{H}^{\mathrm{E} 5}\right), 6.45(\mathrm{~d}$, $\left.J=1.8 \mathrm{~Hz}, 1 \mathrm{H}, \mathrm{H}^{\mathrm{F} 6}\right), 1.05\left(\mathrm{~s}, 9 \mathrm{H}, \mathrm{H}^{\mathrm{tBu}}\right) .{ }^{13} \mathrm{C}\left\{{ }^{1} \mathrm{H}\right\}$ NMR $(126$ $\left.\mathrm{MHz}, \mathrm{CD}_{3} \mathrm{CN}\right) \delta / \mathrm{ppm}: 193.9\left(\mathrm{C}^{\mathrm{F} 1}\right), 167.7\left(\mathrm{C}^{\mathrm{E} 2}\right), 158.7\left(\mathrm{C}^{\mathrm{B} 2}\right)$, $157.9\left(\mathrm{C}^{\mathrm{C} 2}\right), 157.6\left(\mathrm{C}^{\mathrm{D} 2}\right), 156.1\left(\mathrm{C}^{\mathrm{A} 2}\right), 155.0\left(\mathrm{C}^{\mathrm{B} 6}\right), 151.4\left(\mathrm{C}^{\mathrm{E} 6}\right)$, $151.2\left(\mathrm{C}^{\mathrm{D} 6}\right), 151.0\left(\mathrm{C}^{\mathrm{C} 6}\right), 150.3\left(\mathrm{C}^{\mathrm{A} 6}\right), 146.9\left(\mathrm{C}^{\mathrm{F} 2}\right), 144.3\left(\mathrm{C}^{\mathrm{F} 6}\right)$, $137.4\left(\mathrm{C}^{\mathrm{A} 4}\right), 136.8\left(\mathrm{C}^{\mathrm{E} 4}\right), 136.0\left(\mathrm{C}^{\mathrm{B} 4}\right), 134.9\left(\mathrm{C}^{\mathrm{C} 4}\right), 134.7\left(\mathrm{C}^{\mathrm{D} 4}\right)$, $134.0\left(\mathrm{C}^{\mathrm{F} 5}\right), 130.0\left(\mathrm{C}^{\mathrm{F} 4}\right), 128.0\left(\mathrm{C}^{\mathrm{A} 5}\right), 127.4\left(\mathrm{C}^{\mathrm{C} 5}\right), 127.2\left(\mathrm{C}^{\mathrm{B} 5}\right)$, $127.1\left(\mathrm{C}^{\mathrm{D} 5}\right), 124.8\left(\mathrm{C}^{\mathrm{F} 3}\right), 124.5\left(\mathrm{C}^{\mathrm{B} 3}\right), 124.2\left(\mathrm{C}^{\mathrm{A} 3}\right), 124.0\left(\mathrm{C}^{\mathrm{C} 3}\right)$, $123.9\left(\mathrm{C}^{\mathrm{D} 3}\right), 123.6\left(\mathrm{C}^{\mathrm{E} 5}\right), 120.2\left(\mathrm{C}^{\mathrm{E} 3}\right), 46.2\left(\mathrm{C}^{\mathrm{SCMe}}\right), 31.3$ $\left(\mathrm{C}^{\mathrm{tBu}}\right)$. ESI-MS $\mathrm{m} / z$ : $656.4\left[\mathrm{M}-\mathrm{PF}_{6}\right]^{+}$(calc. 656.1). UV-Vis $\left(\mathrm{MeCN}, 1 \times 10^{-5} \mathrm{M}\right) \lambda / \mathrm{nm}\left(\varepsilon / \mathrm{dm}^{3} \mathrm{~mol}^{-1} \mathrm{~cm}^{-1}\right): 249$ (34500), 296 (65400), 362 (12200), 395 (10100), 492 (9900), 541 (10500). Emission (MeCN, $5 \times 10^{-5} \mathrm{M}, \lambda_{\mathrm{exc}}=570 \mathrm{~nm}$ ): $\lambda_{\max }^{\mathrm{em}}$ $=792 \mathrm{~nm}$. Found $\mathrm{C} 52.19, \mathrm{H} 4.41, \mathrm{~N} 8.61 ; \mathrm{C}_{35} \mathrm{H}_{32} \mathrm{~F}_{6} \mathrm{~N}_{5} \mathrm{PRuS}$ requires C 52.50, H 4.03, N 8.75.

\section{$\left[\mathbf{R u}(\mathrm{bpy})_{2}(6)\right]\left[\mathrm{PF}_{6}\right]$}

cis-[Ru(bpy $\left.)_{2} \mathrm{Cl}_{2}\right](50.0 \mathrm{mg}, 0.103 \mathrm{mmol}), \mathrm{H} 6(52.0 \mathrm{mg}, 0.223$ $\mathrm{mmol})$ and $\mathrm{AgBF}_{4}(46.0 \mathrm{mg}, 0.236 \mathrm{mmol})$ were dissolved in $\mathrm{CH}_{2} \mathrm{Cl}_{2}(7.5 \mathrm{~mL})$ and the dark purple solution heated at reflux for $24 \mathrm{~h}$. After filtration through Celite, the volume of the solution was reduced and $n$-hexane $(50 \mathrm{~mL})$ was added to the dark purple oily residue; the mixture was left in the refrigerator 
overnight. The solution was decanted and the dark purple-red precipitate dried under reduced pressure. The residue was dissolved in $\mathrm{MeCN}(10 \mathrm{~mL})$ and an excess of aqueous $\mathrm{NH}_{4} \mathrm{PF}_{6}$ was added. The precipitate was separated by filtration, washed with $\mathrm{H}_{2} \mathrm{O}$ and $\mathrm{Et}_{2} \mathrm{O}$ and redissolved with $\mathrm{CH}_{2} \mathrm{Cl}_{2}$. The solution was concentrated under reduced pressure and purified by flash chromatography $\left(\mathrm{Al}_{2} \mathrm{O}_{3}\right.$, acetone/toluene 1:1 changing to acetone). $\left[\mathrm{Ru}(\mathrm{bpy})_{2}(\mathbf{6})\right]\left[\mathrm{PF}_{6}\right]$ was isolated as a dark purple solid (23 mg, $0.029 \mathrm{mmol}, 28 \%) .{ }^{1} \mathrm{H}$ NMR (500 $\left.\mathrm{MHz}, \mathrm{CD}_{3} \mathrm{CN}\right)$ $\delta /$ ppm: $8.46\left(\mathrm{dt}, J=8.3,1.0 \mathrm{~Hz}, 1 \mathrm{H}, \mathrm{H}^{\mathrm{A} 3}\right), 8.39(\mathrm{dt}, J=8.1,1.0$ $\left.\mathrm{Hz}, 1 \mathrm{H}, \mathrm{H}^{\mathrm{B} 3}\right), 8.36\left(\mathrm{dt}, J=8.2,1.1 \mathrm{~Hz}, 1 \mathrm{H}, \mathrm{H}^{\mathrm{C} 3}\right), 8.32(\mathrm{dt}, J=$ $\left.8.2,1.0 \mathrm{~Hz}, 1 \mathrm{H}, \mathrm{H}^{\mathrm{D} 3}\right), 8.15\left(\mathrm{dt}, J=8.3,1.0 \mathrm{~Hz}, 1 \mathrm{H}, \mathrm{H}^{\mathrm{E} 3}\right), 8.01$ $\left(\mathrm{d}, J=8.2 \mathrm{~Hz}, 1 \mathrm{H}, \mathrm{H}^{\mathrm{F} 3}\right), 8.00(\mathrm{ddd}, J=8.2,7.6,1.6 \mathrm{~Hz}, 1 \mathrm{H}$, $\left.\mathrm{H}^{\mathrm{A} 4}\right), 7.97\left(\mathrm{ddd}, J=5.7,1.6,0.7 \mathrm{~Hz}, 1 \mathrm{H}, \mathrm{H}^{\mathrm{B} 6}\right), 7.87(\mathrm{ddd}, J=$ $\left.8.2, \quad 7.6, \quad 1.5 \mathrm{~Hz}, \quad 1 \mathrm{H}, \quad \mathrm{H}^{\mathrm{C} 4}\right), \quad 7.85-7.73 \quad(\mathrm{~m}, \quad 6 \mathrm{H}, \quad \mathrm{H}$ B4+A6+D4+E4+D6+C6 $), 7.65\left(\mathrm{ddd}, J=5.7,1.6,0.8 \mathrm{~Hz}, 1 \mathrm{H}, \mathrm{H}^{\mathrm{E}}\right), 7.42$ (ddd, $\left.J=7.6,5.4,1.2 \mathrm{~Hz}, 1 \mathrm{H}, \mathrm{H}^{\mathrm{A} 5}\right), 7.38(\mathrm{dd}, J=8.2,2.0 \mathrm{~Hz}$, $\left.1 \mathrm{H}, \mathrm{H}^{\mathrm{F} 4}\right), 7.27\left(\mathrm{ddd}, J=7.3,5.7,1.4 \mathrm{~Hz}, 1 \mathrm{H}, \mathrm{H}^{\mathrm{C} 5}\right), 7.24-7.20$ $\left(\mathrm{m}, 2 \mathrm{H}, \mathrm{H}^{\mathrm{D} 5+\mathrm{B} 5}\right), 7.04\left(\mathrm{ddd}, J=7.3,5.7,1.3 \mathrm{~Hz}, 1 \mathrm{H}, \mathrm{H}^{\mathrm{E} 5}\right), 6.93$ $\left(\mathrm{d}, J=1.9 \mathrm{~Hz}, 1 \mathrm{H}, \mathrm{H}^{\mathrm{F} 6}\right), 2.84\left(\mathrm{~s}, 3 \mathrm{H}, \mathrm{H}^{\mathrm{Me}}\right) .{ }^{13} \mathrm{C}\left\{{ }^{1} \mathrm{H}\right\} \mathrm{NMR}(126$ $\left.\mathrm{MHz}, 295 \mathrm{~K}, \mathrm{CD}_{3} \mathrm{CN}\right) \delta / \mathrm{ppm}: 196.2\left(\mathrm{C}^{\mathrm{F} 1}\right), 166.5\left(\mathrm{C}^{\mathrm{E} 2}\right), 158.5$ $\left(\mathrm{C}^{\mathrm{B} 2}\right), 157.8\left(\mathrm{C}^{\mathrm{C} 2}\right), 157.6\left(\mathrm{C}^{\mathrm{D} 2}\right), 156.1\left(\mathrm{C}^{\mathrm{A} 2}\right), 155.3\left(\mathrm{C}^{\mathrm{B} 6}\right), 152.1$ $\left(\mathrm{C}^{\mathrm{F} 2}\right), 151.8\left(\mathrm{C}^{\mathrm{E} 6}\right), 151.4\left(\mathrm{C}^{\mathrm{D} 6}\right), 151.2\left(\mathrm{C}^{\mathrm{C} 6}\right), 150.2\left(\mathrm{C}^{\mathrm{A} 6}\right), 140.3$ $\left(\mathrm{C}^{\mathrm{F} 5}\right), 137.6\left(\mathrm{C}^{\mathrm{A} 4}\right), 137.0\left(\mathrm{C}^{\mathrm{E} 4}\right), 136.2\left(\mathrm{C}^{\mathrm{B} 4}\right), 135.4\left(\mathrm{C}^{\mathrm{C} 4}\right), 135.3$ $\left(\mathrm{C}^{\mathrm{D} 4}\right), 133.4\left(\mathrm{C}^{\mathrm{F} 6}\right), 128.1\left(\mathrm{C}^{\mathrm{A} 5}\right), 127.6\left(\mathrm{C}^{\mathrm{C} 5}\right), 127.5\left(\mathrm{C}^{\mathrm{B} 5}\right), 127.3$ $\left(\mathrm{C}^{\mathrm{D} 5}\right), 124.9\left(\mathrm{C}^{\mathrm{F} 3}\right), 124.8\left(\mathrm{C}^{\mathrm{E} 5}\right), 124.5\left(\mathrm{C}^{\mathrm{B} 3}\right), 124.3\left(\mathrm{C}^{\mathrm{A3}}\right), 124.2$ $\left(\mathrm{C}^{\mathrm{D} 3}\right), 124.1\left(\mathrm{C}^{\mathrm{C} 3}\right), 121.3\left(\mathrm{C}^{\mathrm{E} 3}\right), 120.1\left(\mathrm{C}^{\mathrm{F} 4}\right), 44.5\left(\mathrm{C}^{\mathrm{Me}}\right)$. ESIMS $m / z$ : $646.4\left[\mathrm{M}-\mathrm{PF}_{6}\right]^{+}$(calc. 646.1). UV-Vis $\left(\mathrm{MeCN}, 1 \times 10^{-}\right.$ ${ }^{5}$ M) $\lambda / \mathrm{nm}\left(\varepsilon / \mathrm{dm}^{3} \mathrm{~mol}^{-1} \mathrm{~cm}^{-1}\right): 248$ (28400), 295 (58100), 358 (10100), 393 (6900), 485 (8500), 530 (8700). Emission (MeCN, $\left.5 \times 10^{-5} \mathrm{M}, \lambda_{\mathrm{exc}}=555 \mathrm{~nm}\right): \lambda_{\max }{ }^{\mathrm{em}}=760 \mathrm{~nm}$. Found $\mathrm{C}$ 48.57, H 3.99, N 8.38; $\mathrm{C}_{32} \mathrm{H}_{26} \mathrm{~F}_{6} \mathrm{~N}_{5} \mathrm{O}_{2}$ PRuS requires $\mathrm{C} 48.61, \mathrm{H}$ $3.31, \mathrm{~N} 8.86$.

\section{$\left[\operatorname{Ru}(\mathbf{b p y})_{2}(7)\right]\left[\mathrm{PF}_{6}\right]$}

cis-[Ru(bpy $\left.)_{2} \mathrm{Cl}_{2}\right](103 \mathrm{mg}, 0.213 \mathrm{mmol}), \mathrm{H} 7$ (100 mg, 0.469 mmol) and $\mathrm{AgPF}_{6}(119 \mathrm{mg}, 0.469 \mathrm{mmol})$ were dissolved in $\mathrm{CH}_{2} \mathrm{Cl}_{2} / \mathrm{MeOH}(3: 1,16 \mathrm{~mL})$ and the solution heated at reflux for $4 \mathrm{~h}$. The mixture was filtered through Celite and the filtrate volume reduced before flash chromatography $\left(\mathrm{Al}_{2} \mathrm{O}_{3}\right.$, acetone/pentane 1:2 changing to acetone). The fractions were concentrated and $n$-hexane was added to precipitate dark purple solid $\left[\mathrm{Ru}(\mathrm{bpy})_{2}(7)\right]\left[\mathrm{PF}_{6}\right]$ (104 mg, $\left.0.135 \mathrm{mmol}, 63 \%\right) .{ }^{1} \mathrm{H} \mathrm{NMR}$ $\left(500 \mathrm{MHz}, 295 \mathrm{~K}, \mathrm{CD}_{3} \mathrm{CN}\right) \delta / \mathrm{ppm}: 8.46(\mathrm{dt}, J=8.2,1.1 \mathrm{~Hz}$, $\left.1 \mathrm{H}, \mathrm{H}^{\mathrm{A} 3}\right), 8.43\left(\mathrm{dd}, J=1.8,0.7 \mathrm{~Hz}, 1 \mathrm{H}, \mathrm{H}^{\mathrm{E} 3}\right), 8.38(\mathrm{ddd}, J=$ $8.2,1.4,0.8 \mathrm{~Hz}, 1 \mathrm{H}, \mathrm{H}^{\mathrm{B} 3}$ ), $8.34(\mathrm{ddd}, J=8.3,1.4,0.8 \mathrm{~Hz}, 1 \mathrm{H}$, $\left.\mathrm{H}^{\mathrm{C} 3}\right), 8.30\left(\mathrm{ddd}, J=8.2,1.4,0.8 \mathrm{~Hz}, 1 \mathrm{H}, \mathrm{H}^{\mathrm{D} 3}\right), 7.98(\mathrm{~m}, 2 \mathrm{H}$, $\left.\mathrm{H}^{\mathrm{B} 6+\mathrm{A} 4}\right), 7.95\left(\mathrm{dd}, J=7.7,1.2 \mathrm{~Hz}, 1 \mathrm{H}, \mathrm{H}^{\mathrm{F} 3}\right), 7.86-7.77(\mathrm{~m}, 5 \mathrm{H}$, $\mathrm{H}^{\mathrm{C} 4+\mathrm{A} 6+\mathrm{B} 4+\mathrm{E} 6+\mathrm{D} 4}$ ), 7.74 (ddd, $\left.J=5.7,1.5,0.8 \mathrm{~Hz}, 1 \mathrm{H}, \mathrm{H}^{\mathrm{D} 6}\right), 7.71$ (ddd, $J=5.7,1.5,0.8 \mathrm{~Hz}, 1 \mathrm{H}, \mathrm{H}^{\mathrm{C} 6}$ ), 7.42 (ddd, $J=7.6,5.4,1.2$ $\left.\mathrm{Hz}, 1 \mathrm{H}, \mathrm{H}^{\mathrm{A} 5}\right), 7.33\left(\mathrm{dd}, J=5.9,1.9 \mathrm{~Hz}, 1 \mathrm{H}, \mathrm{H}^{\mathrm{E} 5}\right), 7.24(\mathrm{ddd}, J=$ 7.5, 5.7, 1.4 Hz, 1H, $\left.\mathrm{H}^{\mathrm{C} 5}\right), 7.19\left(\mathrm{~m}, 2 \mathrm{H}, \mathrm{H}^{\mathrm{B} 5+\mathrm{D} 5}\right), 6.93(\mathrm{ddd}, J=$ $\left.7.8,7.2,1.3 \mathrm{~Hz}, 1 \mathrm{H}, \mathrm{H}^{\mathrm{F} 4}\right), 6.86\left(\mathrm{td}, J=7.3,1.4 \mathrm{~Hz}, 1 \mathrm{H}, \mathrm{H}^{\mathrm{F} 5}\right)$, 6.47 (ddd, $\left.J=7.4,1.3,0.6 \mathrm{~Hz}, 1 \mathrm{H}, \mathrm{H}^{\mathrm{F} 6}\right), 3.91\left(\mathrm{~s}, 3 \mathrm{H}, \mathrm{H}^{\mathrm{Me}}\right)$. ${ }^{13} \mathrm{C}\left\{{ }^{1} \mathrm{H}\right\}$ NMR $\left(126 \mathrm{MHz}, \mathrm{CD}_{3} \mathrm{CN}\right) \delta / \mathrm{ppm}: 194.4\left(\mathrm{C}^{\mathrm{F} 1}\right), 169.7$ $\left(\mathrm{C}^{\mathrm{E} 2}\right), 163.4\left(\mathrm{C}_{2}^{\mathrm{CO}} \mathrm{Me}\right), 158.6\left(\mathrm{C}^{\mathrm{B} 2}\right), 157.4\left(\mathrm{C}^{\mathrm{D} 2}\right), 157.5\left(\mathrm{C}^{\mathrm{C} 2}\right)$,
$156.0\left(\mathrm{C}^{\mathrm{A} 2}\right), 155.3\left(\mathrm{C}^{\mathrm{B} 6}\right), 152.3\left(\mathrm{C}^{\mathrm{E} 6}\right), 151.3\left(\mathrm{C}^{\mathrm{D} 6}\right), 150.9\left(\mathrm{C}^{\mathrm{C} 6}\right)$, $150.1\left(\mathrm{C}^{\mathrm{A} 6}\right), 145.9\left(\mathrm{C}^{\mathrm{F} 2}\right), 137.6\left(\mathrm{C}^{\mathrm{A4}}\right), 137.0\left(\mathrm{C}^{\mathrm{E} 4}\right), 136.32$ $\left(\mathrm{C}^{\mathrm{F} 6}\right), 136.28\left(\mathrm{C}^{\mathrm{B} 4}\right), 135.2\left(\mathrm{C}^{\mathrm{C} 4}\right), 134.9\left(\mathrm{C}^{\mathrm{D} 4}\right), 129.9\left(\mathrm{C}^{\mathrm{F} 5}\right)$, $128.1\left(\mathrm{C}^{\mathrm{A} 5}\right), 127.5\left(\mathrm{C}^{\mathrm{C} 5}\right), 127.4\left(\mathrm{C}^{\mathrm{B} 5}\right), 127.1\left(\mathrm{C}^{\mathrm{D} 5}\right), 125.7\left(\mathrm{C}^{\mathrm{F} 3}\right)$, $124.5\left(\mathrm{C}^{\mathrm{B} 3}\right), 124.2\left(\mathrm{C}^{\mathrm{A} 3}\right), 124.1\left(\mathrm{C}^{\mathrm{D} 3}\right), 124.0\left(\mathrm{C}^{\mathrm{C} 3}\right), 122.0\left(\mathrm{C}^{\mathrm{F} 4}\right)$, $121.7\left(\mathrm{C}^{\mathrm{E} 5}\right), 118.4\left(\mathrm{C}^{\mathrm{E} 3}\right), 53.4\left(\mathrm{C}_{2}^{\mathrm{CO}}{ }_{2}^{M e}\right)$. ESI-MS $m / z: 626.4$ $\left[\mathrm{M}-\mathrm{PF}_{6}\right]^{+}$(calc. 626.1). High resolution ESI-MS $m / z$ : 626.1132 (calc. 626.1133). UV-Vis $\left(\mathrm{MeCN}, 1 \times 10^{-5} \mathrm{M}\right) \lambda / \mathrm{nm}\left(\varepsilon / \mathrm{dm}^{3}\right.$ $\left.\mathrm{mol}^{-1} \mathrm{~cm}^{-1}\right): 250$ (34800), 296 (55100), 322 (10400), 368 (8800), 438 (9100), 499 (12800). Emission (MeCN, $5 \times 10^{-5} \mathrm{M}$, $\left.\lambda_{\text {exc }}=575 \mathrm{~nm}\right): \lambda_{\max }{ }^{\mathrm{em}}=784 \mathrm{~nm}$. Found C 50.44, H 3.75, N 8.77; $\mathrm{C}_{33} \mathrm{H}_{26} \mathrm{~F}_{6} \mathrm{~N}_{5} \mathrm{O}_{2} \mathrm{PRu} \cdot \mathrm{H}_{2} \mathrm{O}$ requires C 50.26, H 3.58, N 8.88.

\section{$\left[\operatorname{Ru}(\mathbf{b p y})_{2}(8)\right]$}

The compound was prepared adapting a previously described procedure for similar complexes. ${ }^{11}\left[\mathrm{Ru}(\mathrm{bpy})_{2}(7)\right]\left[\mathrm{PF}_{6}\right](20 \mathrm{mg}$, $0.0260 \mathrm{mmol})$ was dissolved in $\mathrm{DMF} / \mathrm{H}_{2} \mathrm{O} / \mathrm{NEt}_{3}(3: 1: 1,12 \mathrm{~mL})$ and heated at reflux for $16 \mathrm{~h}$. The solvent was removed and product passed through a short silica plug using $\mathrm{MeOH} / \mathrm{CH}_{2} \mathrm{Cl}_{2}$ $1: 1$. The volume of solution was reduced and was layered with $\mathrm{Et}_{2} \mathrm{O}$. After a few days, $\left[\mathrm{Ru}(\mathrm{bpy})_{2}(\mathbf{8})\right]$ precipitated as a dark purple crystalline solid (14.2 $\mathrm{mg}, 0.0233 \mathrm{mmol}, 89 \%) .{ }^{1} \mathrm{H}$ NMR $\left(500 \mathrm{MHz}, \mathrm{CD}_{3} \mathrm{CN}\right) \delta / \mathrm{ppm}: 8.44\left(\mathrm{~m}, 2 \mathrm{H}, \mathrm{H}^{\mathrm{A} 3+\mathrm{E} 3}\right), 8.37(\mathrm{~d}, J=$ $\left.8.2 \mathrm{~Hz}, 1 \mathrm{H}, \mathrm{H}^{\mathrm{B} 3}\right), 8.31\left(\mathrm{~d}, J=8.3 \mathrm{~Hz}, 1 \mathrm{H}, \mathrm{H}^{\mathrm{C} 3}\right), 8.28(\mathrm{~d}, J=8.4$ $\left.\mathrm{Hz}, 1 \mathrm{H}, \mathrm{H}^{\mathrm{D} 3}\right), 8.07\left(\mathrm{~d}, J=5.7 \mathrm{~Hz}, 1 \mathrm{H}, \mathrm{H}^{\mathrm{B} 6}\right), 7.96(\mathrm{t}, J=7.6 \mathrm{~Hz}$, $\left.1 \mathrm{H}, \mathrm{H}^{\mathrm{A} 4}\right), 7.89\left(\mathrm{~d}, J=7.8 \mathrm{~Hz}, 1 \mathrm{H}, \mathrm{H}^{\mathrm{F} 3}\right), 7.84-7.71(\mathrm{~m}, 6 \mathrm{H}, \mathrm{H}$ A6+D6+C4+B4+D4+C6 $), 7.47\left(\mathrm{~d}, J=5.8 \mathrm{~Hz}, 1 \mathrm{H}, \mathrm{H}^{\mathrm{E} 6}\right), 7.39(\mathrm{t}, J=6.5$ $\left.\mathrm{Hz}, 1 \mathrm{H}, \mathrm{H}^{\mathrm{A} 5}\right), 7.35\left(\mathrm{dd}, J=5.6,1.4 \mathrm{~Hz}, 1 \mathrm{H}, \mathrm{H}^{\mathrm{E} 5}\right), 7.21-7.15(\mathrm{~m}$, $\left.2 \mathrm{H}, \mathrm{H}^{\mathrm{C} 5+\mathrm{B} 5+\mathrm{D} 5}\right), 6.85\left(\mathrm{t}, J=7.4 \mathrm{~Hz}, 1 \mathrm{H}, \mathrm{H}^{\mathrm{F} 4}\right), 6.79(\mathrm{t}, J=7.3$, $\left.1 \mathrm{H}, \mathrm{H}^{\mathrm{F} 5}\right), 6.39\left(\mathrm{~d}, J=7.3 \mathrm{~Hz}, 1 \mathrm{H}, \mathrm{H}^{\mathrm{F} 6}\right) .{ }^{13} \mathrm{C}\left\{{ }^{1} \mathrm{H}\right\}$ NMR $(126$ $\left.\mathrm{MHz}, \mathrm{CD}_{3} \mathrm{CN}\right) \delta / \mathrm{ppm}: 192.9\left(\mathrm{C}^{\mathrm{F} 1}\right), 167.6\left(\mathrm{C}^{\mathrm{E} 2}\right), 158.6\left(\mathrm{C}^{\mathrm{B} 2}\right)$, $157.8\left(\mathrm{C}^{\mathrm{C} 2}\right), 157.7\left(\mathrm{C}^{\mathrm{D} 2}\right), 156.2\left(\mathrm{C}^{\mathrm{A} 2}\right), 154.9\left(\mathrm{C}^{\mathrm{B} 6}\right), 151.3\left(\mathrm{C}^{\mathrm{D} 6}\right)$, $151.0\left(\mathrm{C}^{\mathrm{C} 6}\right), 150.4\left(\mathrm{C}^{\mathrm{E} 6}\right), 150.3\left(\mathrm{C}^{\mathrm{CO}}\right), 150.0\left(\mathrm{C}^{\mathrm{A} 6}\right), 149.8$ $\left(\mathrm{C}^{\mathrm{E} 4}\right), 147.2\left(\mathrm{C}^{\mathrm{F} 2}\right), 137.1\left(\mathrm{C}^{\mathrm{A} 4}\right), 136.0\left(\mathrm{C}^{\mathrm{F} 6}\right), 135.7\left(\mathrm{C}^{\mathrm{B} 4}\right), 134.5$ $\left(\mathrm{C}^{\mathrm{C} 4}\right), 134.2\left(\mathrm{C}^{\mathrm{D} 4}\right), 129.0\left(\mathrm{C}^{\mathrm{F} 5}\right), 127.9\left(\mathrm{C}^{\mathrm{A} 5}\right), 127.2\left(\mathrm{C}^{\mathrm{C} 5}\right), 127.1$ $\left(\mathrm{C}^{\mathrm{B} 5}\right), 127.0\left(\mathrm{C}^{\mathrm{D} 5}\right), 125.0\left(\mathrm{C}^{\mathrm{F} 3}\right), 124.3\left(\mathrm{C}^{\mathrm{B} 3}\right), 124.1\left(\mathrm{C}^{\mathrm{A} 3}\right), 123.8$ $\left(2 \mathrm{C}, \mathrm{C}^{\mathrm{D} 3+\mathrm{C} 3}\right), 123.3\left(\mathrm{C}^{\mathrm{E} 5}\right), 121.8\left(\mathrm{C}^{\mathrm{F} 4}\right), 119.3\left(\mathrm{C}^{\mathrm{E} 3}\right)$. MALDITOF MS (matrix: 4-nitroaniline) $\mathrm{m} / z: 612.3[\mathrm{M}+\mathrm{H}]^{+}$(calc. 612.1), $568.3\left[\mathrm{M}+\mathrm{H}-\mathrm{CO}_{2}\right]^{+}$(calc. 568.1), $456.1[\mathrm{M}+\mathrm{H}-\text { bpy }]^{+}$ (calc. 456.0). High resolution ESI-MS $m / z$ : 612.0967 (calc. 612.0976). UV-Vis $\left(\mathrm{MeCN}, 1 \times 10^{-5} \mathrm{M}\right) \lambda / \mathrm{nm}\left(\varepsilon / \mathrm{dm}^{3} \mathrm{~mol}^{-1}\right.$ $\mathrm{cm}^{-1}$ ): 249 (34500), 297 (48400), 365 (10800), 408 (10400), 492 (7100), 551 (7500). Emission ( $\mathrm{MeCN}, 5 \times 10^{-5} \mathrm{M}, \lambda_{\mathrm{exc}}=$ $580 \mathrm{~nm}): \lambda_{\max }^{\mathrm{em}}=809 \mathrm{~nm}$. Satisfactory elemental analysis could not be obtained.

\section{$\left[\operatorname{Ru}(\operatorname{bpy})_{2}(\mathbf{9})\right]\left[\mathrm{PF}_{6}\right]$}

cis-[Ru(bpy $\left.)_{2} \mathrm{Cl}_{2}\right](116 \mathrm{mg}, 0.240 \mathrm{mmol}), \mathrm{H} 9$ (194 mg, 0.528 $\mathrm{mmol})$ and $\mathrm{AgPF}_{6}(133 \mathrm{mg}, 0.528 \mathrm{mmol})$ were dissolved in $\mathrm{CH}_{2} \mathrm{Cl}_{2}(15 \mathrm{~mL})$ and the solution heated at reflux for $16 \mathrm{~h}$. After filtration through Celite, the volume of solution was reduced before flash chromatography $\left(\mathrm{Al}_{2} \mathrm{O}_{3}\right.$, acetone/pentane 1:2 changing to acetone). The fractions were combined, the volume of solvent reduced, and $n$-hexane added to precipitate dark purple $\left[\mathrm{Ru}(\mathrm{bpy})_{2}(\mathbf{1 1})\right]\left[\mathrm{PF}_{6}\right](127 \mathrm{mg}, 0.137 \mathrm{mmol}, 64 \%)$. ${ }^{1} \mathrm{H}$ NMR (500 MHz, $\mathrm{CD}_{3} \mathrm{CN}$ ) $\delta / \mathrm{ppm}: 8.47(\mathrm{dt}, J=8.3,1.1 \mathrm{~Hz}$, 
$\left.1 \mathrm{H}, \mathrm{H}^{\mathrm{A} 3}\right), 8.40\left(\mathrm{ddd}, J=8.3,1.4,0.8 \mathrm{~Hz}, 1 \mathrm{H}, \mathrm{H}^{\mathrm{B} 3}\right), 8.33(\mathrm{dt}, J=$ $\left.8.3,1.3 \mathrm{~Hz}, 1 \mathrm{H}, \mathrm{H}^{\mathrm{C} 3}\right), 8.31\left(\mathrm{dt}, J=8.3,1.1 \mathrm{~Hz}, 1 \mathrm{H}, \mathrm{H}^{\mathrm{D} 3}\right), 8.33$ $\left(\mathrm{dd}, J=2.1,0.7 \mathrm{~Hz}, 1 \mathrm{H}, \mathrm{H}^{\mathrm{E} 3}\right), 8.08(\mathrm{ddd}, J=5.7,1.6,0.8 \mathrm{~Hz}$, $\left.1 \mathrm{H}, \mathrm{H}^{\mathrm{B} 6}\right), 8.00\left(\mathrm{~m}, 2 \mathrm{H}, \mathrm{H}^{\mathrm{F} 3+\mathrm{A} 4}\right), 7.90\left(\mathrm{~m}, 4 \mathrm{H}, \mathrm{H}^{\mathrm{G} 3+\mathrm{G} 2}\right), 7.83(\mathrm{~m}$, $\left.4 \mathrm{H}, \mathrm{H}^{\mathrm{A} 6+\mathrm{D} 6+\mathrm{C} 4+\mathrm{B} 4}\right), 7.78\left(\mathrm{ddd}, J=8.2,7.5,1.5 \mathrm{~Hz}, 1 \mathrm{H}, \mathrm{H}^{\mathrm{D} 4}\right)$, $7.74\left(\mathrm{ddd}, J=5.7,1.5,0.8 \mathrm{~Hz}, 1 \mathrm{H}, \mathrm{H}^{\mathrm{C} 6}\right), 7.64(\mathrm{dd}, J=6.0,0.7$ $\left.\mathrm{Hz}, 1 \mathrm{H}, \mathrm{H}^{\mathrm{E} 6}\right), 7.43\left(\mathrm{ddd}, J=7.6,5.4,1.2 \mathrm{~Hz}, 1 \mathrm{H}, \mathrm{H}^{\mathrm{A} 5}\right), 7.22(\mathrm{~m}$, $\left.4 \mathrm{H}, \mathrm{H}^{\mathrm{D} 5+\mathrm{C} 5+\mathrm{E} 5+\mathrm{B} 5}\right), 6.92\left(\mathrm{ddd}, J=7.7,7.2,1.3 \mathrm{~Hz}, 1 \mathrm{H}, \mathrm{H}^{\mathrm{F} 4}\right.$ ), $6.85\left(\mathrm{td}, J=7.3,1.3 \mathrm{~Hz}, 1 \mathrm{H}, \mathrm{H}^{\mathrm{F} 5}\right), 6.46(\mathrm{ddd}, J=7.3,1.3,0.6$ $\left.\mathrm{Hz}, 1 \mathrm{H}, \mathrm{H}^{\mathrm{F} 6}\right), 4.08\left(\mathrm{~m}, 4 \mathrm{H}, \mathrm{H}^{\mathrm{PO}\left(\mathrm{OCH}_{2} \mathrm{Me}_{2}\right)}\right), 1.29(\mathrm{~m}, 6 \mathrm{H}$, $\left.\mathrm{H}^{\mathrm{PO}(\mathrm{OCH}}{ }_{2}{ }_{2 e}\right) .{ }^{13} \mathrm{C}\left\{{ }^{1} \mathrm{H}\right\}$ NMR $\left(126 \mathrm{MHz}, \mathrm{CD}_{3} \mathrm{CN}\right) \delta / \mathrm{ppm}: 193.8$ $\left(\mathrm{C}^{\mathrm{F} 1}\right), 168.9\left(\mathrm{C}^{\mathrm{E} 2}\right), 158.7\left(\mathrm{C}^{\mathrm{B} 2}\right), 157.72\left(\mathrm{C}^{\mathrm{C} 2}\right), 157.66\left(\mathrm{C}^{\mathrm{D} 2}\right)$, $156.1\left(\mathrm{C}^{\mathrm{A} 2}\right), 155.2\left(\mathrm{C}^{\mathrm{B} 6}\right), 151.7\left(\mathrm{C}^{\mathrm{E} 6}\right), 151.2\left(\mathrm{C}^{\mathrm{D} 6}\right), 150.9\left(\mathrm{C}^{\mathrm{C} 6}\right)$, $150.2\left(\mathrm{C}^{\mathrm{A} 6}\right), 147.0\left(\mathrm{C}^{\mathrm{E} 4}\right), 146.6\left(\mathrm{C}^{\mathrm{F} 2}\right), 142.1\left(\mathrm{~d}, J_{\mathrm{PC}}=3.2 \mathrm{~Hz}\right.$, $\left.\mathrm{C}^{\mathrm{G} 1}\right), 137.4\left(\mathrm{C}^{\mathrm{A} 4}\right), 136.4\left(\mathrm{C}^{\mathrm{F} 6}\right), 136.0\left(\mathrm{C}^{\mathrm{B} 4}\right), 134.9\left(\mathrm{C}^{\mathrm{C} 4}\right), 134.7$ $\left(\mathrm{C}^{\mathrm{D} 4}\right), 133.2\left(\mathrm{~d}, J_{\mathrm{PC}}=10.0 \mathrm{~Hz}, 2 \mathrm{C}, \mathrm{C}^{\mathrm{G} 2}\right), 130.9\left(\mathrm{~d}, J_{\mathrm{PC}}=188\right.$ $\left.\mathrm{Hz}, \mathrm{C}^{\mathrm{G} 4}\right), 129.5\left(\mathrm{C}^{\mathrm{F} 5}\right), 128.3\left(\mathrm{~d}, J_{\mathrm{PC}}=15.0 \mathrm{~Hz}, 2 \mathrm{C}, \mathrm{C}^{\mathrm{G} 3}\right), 128.1$ $\left(\mathrm{C}^{\mathrm{A} 5}\right), 127.4\left(\mathrm{C}^{\mathrm{C} 5}\right), 127.3\left(\mathrm{C}^{\mathrm{B} 5}\right), 127.0\left(\mathrm{C}^{\mathrm{D} 5}\right), 125.4\left(\mathrm{C}^{\mathrm{F} 3}\right), 124.5$ $\left(\mathrm{C}^{\mathrm{B} 3}\right), 124.2\left(\mathrm{C}^{\mathrm{A} 3}\right), 124.0\left(\mathrm{C}^{\mathrm{D} 3}\right), 123.9\left(\mathrm{C}^{\mathrm{C} 3}\right), 122.0\left(\mathrm{C}^{\mathrm{F} 4}\right), 121.1$ $\left(\mathrm{C}^{\mathrm{E} 5}\right), 117.4\left(\mathrm{C}^{\mathrm{E} 3}\right), 63.1\left(\mathrm{~d}, J_{\mathrm{PC}}=5.6 \mathrm{~Hz}, \mathrm{C}_{2}^{\mathrm{PO}\left(\mathrm{OCH}_{2}{ }_{2}\right)}\right), 16.7(\mathrm{~d}$, $\left.J_{\mathrm{PC}}=6.2 \mathrm{~Hz}, \mathrm{C}^{\mathrm{PO}\left(\mathrm{OCH}_{2} M e\right)}{ }_{2}\right) .{ }^{31} \mathrm{P} \mathrm{NMR}\left(162 \mathrm{MHz}, \mathrm{CD}_{3} \mathrm{CN}\right)$ $\delta /$ ppm: $17.0\left(\mathrm{P}^{P \mathrm{O}(\mathrm{OEt})}{ }_{2}\right),-144.6$ (hept, $\left.J_{\mathrm{PF}}=706 \mathrm{~Hz}, \mathrm{P}^{P \mathrm{~F}}{ }_{6}\right)$. ESIMS $m / z$ : $780.5\left[\mathrm{M}-\mathrm{PF}_{6}\right]^{+}$(calc. 780.2). UV-Vis $\left(\mathrm{MeCN}, 1 \times 10^{-}\right.$ ${ }^{5}$ M) $\lambda / \mathrm{nm}\left(\varepsilon / \mathrm{dm}^{3} \mathrm{~mol}^{-1} \mathrm{~cm}^{-1}\right): 253$ (45700), 297 (53600), 371 (10800), 430 (12800), 491 (11900), 537 (10700). Emission $\left(\mathrm{MeCN}, 5 \times 10^{-5} \mathrm{M}, \lambda_{\text {exc }}=570 \mathrm{~nm}\right): \lambda_{\max }{ }^{\mathrm{em}}=798 \mathrm{~nm}$. Found $\mathrm{C}$ 52.36, $\mathrm{H}$ 4.58, N 7.12; $\mathrm{C}_{41} \mathrm{H}_{37} \mathrm{~F}_{6} \mathrm{~N}_{5} \mathrm{O}_{3} \mathrm{P}_{2} \mathrm{Ru} \cdot \mathrm{H}_{2} \mathrm{O}$ requires $\mathrm{C}$ $52.23, \mathrm{H} 4.17, \mathrm{~N} 7.43$.

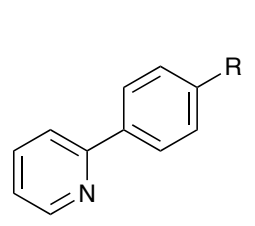

$$
\begin{array}{ll}
\text { Hppy } & R=\mathrm{H} \\
\mathrm{H} 1 & \mathrm{R}=\mathrm{F} \\
\mathrm{H} 2 & \mathrm{R}=\mathrm{Me} \\
\mathrm{H} 3 & \mathrm{R}=\mathrm{OMe} \\
\mathrm{H} 4 & \mathrm{R}=\mathrm{CO}_{2} \mathrm{Me} \\
\mathrm{H} 5 & \mathrm{R}=\mathrm{S}^{\mathrm{t}} \mathrm{Bu} \\
\mathrm{H} 6 & \mathrm{R}=\mathrm{SO}_{2} \mathrm{Me}
\end{array}
$$

Scheme 1. Structure of Hppy and 4-phenyl-functionalized derivatives.

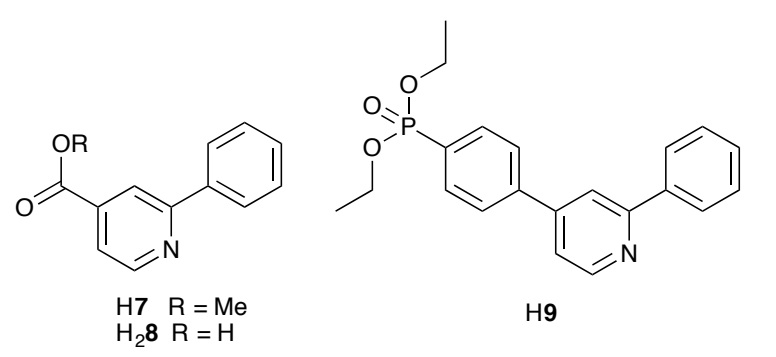

Scheme 2. Structures of cyclometallating ligands with anchoring domains.

\section{Crystallography}

Single crystal data were collected on a Bruker APEX-II diffractometer with data reduction, solution and refinement using the programs APEX, ${ }^{29}$ and CRYSTALS ${ }^{30}$ or SHELX13. ${ }^{31}$ ORTEP diagrams and structure analysis used Mercury v. 3.0. ${ }^{32,33}$ For $\left[\mathrm{Ru}(\mathrm{bpy})_{2}(4)\right]\left[\mathrm{PF}_{6}\right] \cdot \mathrm{MeCN}$, SQUEEZE ${ }^{34}$ was used as one disordered solvent molecule could not be modelled in a satisfactory way.

\section{$\left[\mathrm{Ru}(\mathrm{bpy})_{2}(4)\right]\left[\mathrm{PF}_{6}\right] \cdot \mathrm{MeCN}$}

$\mathrm{C}_{35} \mathrm{H}_{29} \mathrm{~F}_{6} \mathrm{~N}_{6} \mathrm{O}_{2} \mathrm{PRu}, M=811.69$, purple plate, triclinic, space group $P-1, a=10.6299(10), b=13.1587(12), c=14.0271(13)$ $\AA, \alpha=82.634(3), \beta=73.460(3), \gamma=75.358(3)^{\circ}, U=1816.4(3)$ $\AA^{3}, Z=2, D_{c}=1.484 \mathrm{Mg} \mathrm{m}^{-3}, \mu(\mathrm{Cu}-\mathrm{K \alpha} \alpha)=4.534 \mathrm{~mm}^{-1}, T=$ 123 K. Total 22665 reflections, 6412 unique, $R_{\text {int }}=0.034$. Refinement of 6300 reflections (488 parameters) with $I>3 \sigma(I)$ converged at final $R 1=0.0373(R 1$ all data $=0.0377), w R 2=$ $0.0571(w R 2$ all data $=0.0587)$, gof $=1.1954$. CCDC 1011911 .

\section{$\left[\mathbf{R u}(\mathrm{bpy})_{\mathbf{2}}(\mathbf{5})\right]\left[\mathrm{PF}_{\mathbf{6}}\right]$}

$\mathrm{C}_{35} \mathrm{H}_{32} \mathrm{~F}_{6} \mathrm{~N}_{5} \mathrm{PRuS}, M=800.77$, black plate, triclinic, space group $P-1, a=9.1746(6), b=13.7422(9), c=13.9635(9) \AA$, $\alpha=77.596(2), \beta=75.777(2), \gamma=81.750(2)^{\circ}, U=1659.04(19)$ $\AA^{3}, Z=2, D_{c}=1.603 \mathrm{Mg} \mathrm{m}^{-3}, \mu(\mathrm{Cu}-\mathrm{K \alpha})=5.469 \mathrm{~mm}^{-1}, T=$ $123 \mathrm{~K}$. Total 21787 reflections, 5911 unique, $R_{\text {int }}=0.0293$. Refinement of 5747 reflections (445 parameters) with $I>2 \sigma(I)$ converged at final $R 1=0.0243(R 1$ all data $=0.0250), w R 2=$ $0.0618(w R 2$ all data $=0.0622)$, gof $=1.043$. CCDC 1011912 .

\section{$\left[\mathbf{R u}(\mathbf{b p y})_{2}(\mathbf{7})\right]\left[\mathbf{P F}_{6}\right]$}

$\mathrm{C}_{33} \mathrm{H}_{26} \mathrm{~F}_{6} \mathrm{~N}_{5} \mathrm{O}_{2} \mathrm{PRu}, M=770.63$, red needle, monoclinic, space group $P 2_{1} / n, a=12.9211(7), b=16.9306(10), c=15.0031(9)$ $\AA, \beta=108.261(2)^{\circ}, U=3116.8(3) \AA^{3}, Z=4, D_{c}=1.642 \mathrm{Mg} \mathrm{m}^{-}$ ${ }^{3}, \mu(\mathrm{Cu}-\mathrm{K} \alpha)=5.239 \mathrm{~mm}^{-1}, T=123 \mathrm{~K}$. Total 53663 reflections, 5856 unique, $R_{\text {int }}=0.0384$. Refinement of 5340 reflections (435 parameters) with $I>2 \sigma(I)$ converged at final $R 1=0.0248(R 1$ all data $=0.0276), w R 2=0.0642(w R 2$ all data $=0.0664)$, gof $=$ 1.047. CCDC 1011910.

\section{Results and conclusions}

\section{Cyclometallating ligands with anchoring domains}

One convenient method of preparing carboxylic acid and phosphonic acid functionalized bpy and ppy ligands is via deprotection of the corresponding esters. ${ }^{18,35,36}$ The ester $\mathrm{H} 7$ and carboxylic acid $\mathrm{H}_{2} 8$ (Scheme 2) were prepared according to literature methods, and their spectroscopic signatures were in accord with those reported. ${ }^{28}$ The introduction of phenyl spacers between metal-binding and anchoring domains is beneficial in both $n$ - and p-type dyes, ${ }^{14,36}$ and ligand H9 was designed with this in mind. The precursor to H9 was 2-phenyl4-(4-bromophenyl)pyridine which was prepared as shown in Scheme 3. The intermediate 2-phenyl-4-(4bromophenyl)pyridine-6-carboxylic acid was prepared by adapting literature methods for similar compounds. ${ }^{37,38}$ Decarboxylation by heating in ethane-1,2-diol gave the desired product in good yield. The ${ }^{1} \mathrm{H}$ and ${ }^{13} \mathrm{C}$ NMR spectra of a $\mathrm{CDCl}_{3}$ solution of 2-phenyl-4-(4-bromophenyl)pyridine were consistent with the structure shown in Scheme 3. Attempts to obtain an electrospray mass spectrum using a conventional instrument were not successful, but use of an LC-ESI 
combination gave a base peak at $\mathrm{m} / \mathrm{z} 310.0$ arising from the $[\mathrm{M}+\mathrm{H}]^{+}$ion.

Scheme 4 shows the route to phosphonate ester H9 which is based on the method of Odobel and coworkers ${ }^{39}$ and has been successfully applied to prepare a related phosphonic esterfunctionalized bpy containing phenyl spacers. ${ }^{36}$ The base peak in the electrospray mass spectrum of $\mathrm{H} 9$ and the next most intense peak $(\mathrm{m} / \mathrm{z} 390.4$ and 368.4$)$ arose from $[\mathrm{M}+\mathrm{Na}]^{+}$and $[\mathrm{M}+\mathrm{H}]^{+}$, respectively. The ${ }^{1} \mathrm{H}$ and ${ }^{13} \mathrm{C}$ NMR spectra were assigned by $2 \mathrm{D}$ techniques and were consistent with the structure in Scheme 4. The phosphonate ester was characterized in the ${ }^{31} \mathrm{P}\left\{{ }^{1} \mathrm{H}\right\}$ NMR spectrum by a singlet at $\partial-17.1 \mathrm{ppm}$.

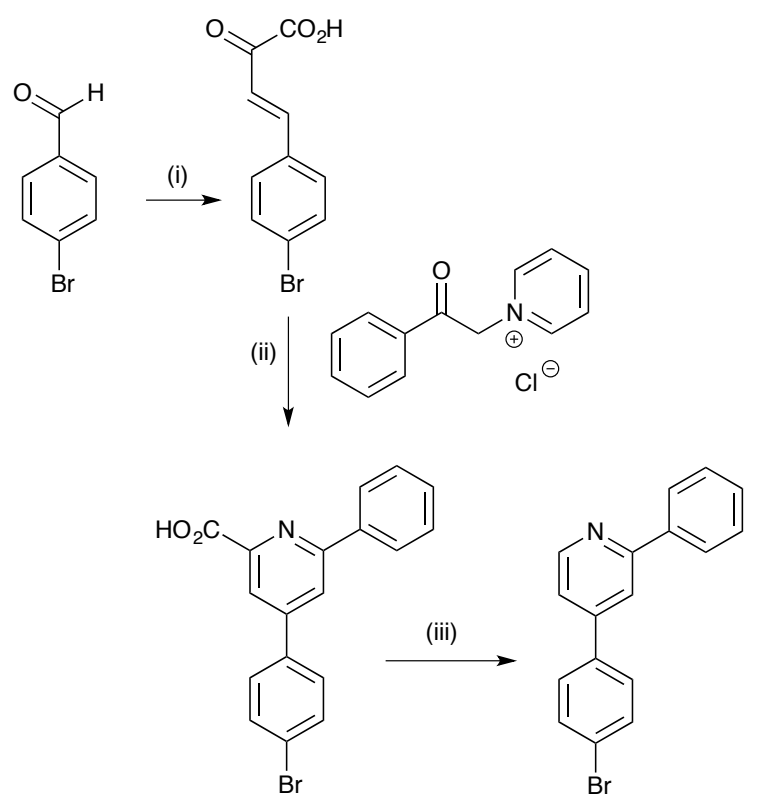

Scheme 3. Synthesis of 2-phenyl-4-(4-bromophenyl)pyridine, precursor to $\mathrm{H} \mathbf{9}$ Conditions: (i) $\mathrm{Na}\left[\mathrm{MeC}(\mathrm{O}) \mathrm{CO}_{2}\right], \mathrm{KOH}, \mathrm{EtOH} / \mathrm{H}_{2} \mathrm{O} 4: 1,0{ }^{\circ} \mathrm{C}, 3 \mathrm{~h}$; (ii) $\mathrm{NH}_{4} \mathrm{OAc}(\mathrm{aq}$ ) $100{ }^{\circ} \mathrm{C}$, 6h; (iii) ethane-1,2-diol, $200{ }^{\circ} \mathrm{C}, 24 \mathrm{~h}$.

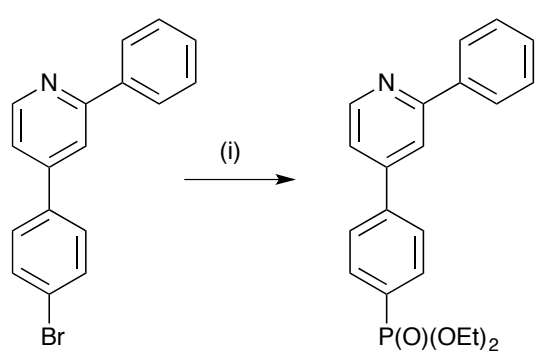

Scheme 4. Synthesis of H9. Conditions: (i) $\left[\mathrm{Pd}\left(\mathrm{PPh}_{3}\right)_{4}\right], \mathrm{Cs}_{2} \mathrm{CO}_{3}, \mathrm{HPO}(\mathrm{OEt})_{2}$, THF, microwave, $110^{\circ} \mathrm{C}, 90 \mathrm{~min}$.

\section{$\left[\operatorname{Ru}(b p y)_{2}\left(C^{\wedge} N\right)\right]\left[P_{6}\right]$ with $C^{\wedge} N=1-6$ : synthesis, $N M R$ spectroscopic and structural characterizations}

The range of $\mathrm{C}^{\wedge} \mathrm{N}$ ligands incorporated both electronwithdrawing and donating groups. The archetype complex $\left[\mathrm{Ru}(\mathrm{bpy})_{2}(\mathrm{ppy})\right]\left[\mathrm{PF}_{6}\right]$ and its derivatives $\left[\mathrm{Ru}(\mathrm{bpy})_{2}\left(\mathrm{C}^{\wedge} \mathrm{N}\right)\right]\left[\mathrm{PF}_{6}\right]$ with $\mathrm{C}^{\wedge} \mathrm{N}=1-6$ were prepared using a strategy reported previously by one of us. ${ }^{1}$ The reaction of $c i s-\left[\mathrm{Ru}(\mathrm{bpy})_{2} \mathrm{Cl}_{2}\right]$ with the cyclometallating ligand is carried out in the presence of a
silver(I) salt (typically $\mathrm{AgPF}_{6}$ or $\left.\mathrm{AgBF}_{4}\right)^{1}$ to abstract the chloride ions. After work up, the cyclometallated complexes were obtained in yields ranging from $26 \%\left(\mathrm{C}^{\wedge} \mathrm{N}=5\right)$ to $60 \%$ $\left(\mathrm{C}^{\wedge} \mathrm{N}=\mathbf{2}\right)$.

The base peak in the ESI mass spectrum of each complex was assigned to $\left[\mathrm{M}-\mathrm{PF}_{6}\right]^{+}$and was dominated by the characteristic isotope pattern of ruthenium. The ${ }^{1} \mathrm{H}$ and ${ }^{13} \mathrm{C}$ NMR spectra of $\mathrm{CD}_{3} \mathrm{CN}$ solutions of the complexes were recorded at room temperature. The spectra were assigned using a combination of COSY, HMQC, HMBC and NOESY methods. The aromatic regions of the ${ }^{1} \mathrm{H}$ NMR spectra of $\left[\mathrm{Ru}(\mathrm{bpy})_{2}\left(\mathrm{C}^{\wedge} \mathrm{N}\right)\right]\left[\mathrm{PF}_{6}\right]$ with $\mathrm{C}^{\wedge} \mathrm{N}=\mathbf{1 - 6}$ were similar and the spectrum of $\left[\mathrm{Ru}(\mathrm{bpy})_{2}(\mathbf{5})\right]\left[\mathrm{PF}_{6}\right]$ is shown in Fig. 1 as a representative example. The presence of the cyclometallating ligand renders the two bpy-ligands inequivalent (Scheme 5). In the NOESY spectrum, cross-peaks observed between the signals for $\mathrm{H}^{\mathrm{B} 6}, \mathrm{H}^{\mathrm{C} 6}$ and $\mathrm{H}^{\mathrm{F} 6}$ as well as between signals for $\mathrm{H}^{\mathrm{A} 6}$, $\mathrm{H}^{\mathrm{D} 6}$ and $\mathrm{H}^{\mathrm{E} 6}$ were especially useful during signal assignment. In Fig. 1, the lowest frequency doublet $(J=1.8 \mathrm{~Hz})$ was assigned to $\mathrm{H}^{\mathrm{F} 6}$. A change in the substituent $\mathrm{R}$ (Scheme 5) most strongly influences the signals for the ring $\mathrm{F}$ protons and to a smaller extent those of ring E. For example, the chemical shift for $\mathrm{H}^{\mathrm{F} 6}$ appears at $\partial 6.41 \mathrm{ppm}$ for $\mathrm{C}^{\wedge} \mathrm{N}=$ ppy, $\partial 6.05 \mathrm{ppm}$ for $\mathbf{1}$ (fluoro substituent), $\partial 6.24 \mathrm{ppm}$ for $2(\mathrm{Me}), \partial 5.84 \mathrm{ppm}$ for $3(\mathrm{OMe}), \partial$ $7.08 \mathrm{ppm}$ for $4\left(\mathrm{CO}_{2} \mathrm{Me}\right), \partial 6.45 \mathrm{ppm}$ for $5\left(\mathrm{~S}^{t} \mathrm{Bu}\right)$, and $\partial 6.93$ ppm for $6\left(\mathrm{SO}_{2} \mathrm{Me}\right)$.

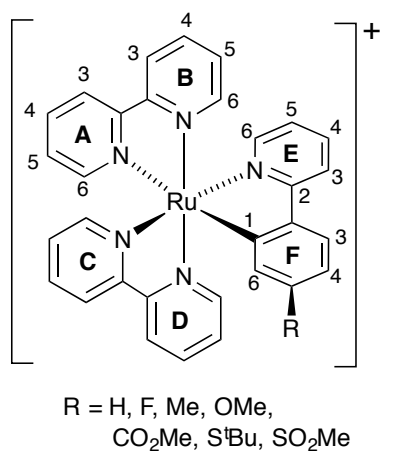

Scheme 5. Atom numbering for NMR spectroscopic assignments in complexes with ligands ppy, 1-6; an analogous scheme is used for complexes with ligands 7 , $\mathbf{8}$ and $\mathbf{9}$ with the phenyl spacer ring labelled $\mathrm{G}$ in ligand $\mathbf{9}$.

The sharp, well resolved spectrum shown in Fig. 1 is typical of a complex which has been recrystallized, or of a sample that has been standing in $\mathrm{MeCN}$ solution for several days followed by filtration through Celite. Immediately after chromatographic workup, the ${ }^{1} \mathrm{H}$ NMR spectrum of several of the $\left[\mathrm{Ru}(\mathrm{bpy})_{2}\left(\mathrm{C}^{\wedge} \mathrm{N}\right)\right]\left[\mathrm{PF}_{6}\right]$ complexes, notably those with ligands 2 and 3 (Me and OMe substituents) exhibited sharp signals for the coordinated bpy ligands and broad signals for the $\mathrm{C}^{\wedge} \mathrm{N}$ ligand. Over time, the signals sharpened (Fig. 2) and very small amounts of a colourless solid were observed in the NMR tube.

One possible explanation is that ion-pairing involving residual chloride ion might be the origin of the phenomenon. However, addition of $\left[{ }^{n} \mathrm{Bu}_{4} \mathrm{~N}\right] \mathrm{Cl}$ led only to shifts in the $\mathrm{H}^{3}$ protons of bpy (Fig. 3), consistent with the established anion- 
binding by these bpy protons in ruthenium(II) ${ }^{40}$ and iridium(III) complexes. ${ }^{41}$ We suggest that interactions between $\mathrm{Ag}^{+}$and the coordinated $\mathrm{C}^{\wedge} \mathrm{N}$ ligand may be responsible for the broadening of the $\mathrm{H}^{\mathrm{E} 5}, \mathrm{H}^{\mathrm{F} 4}$ and $\mathrm{H}^{\mathrm{F} 6}$ proton signals. Interactions between $\mathrm{Ag}^{+}$and an aromatic $\pi$-system are documented and result in similar effects on ${ }^{1} \mathrm{H}$ NMR spectra. ${ }^{42}$ Attempts to gain further insight into the phenomenon were unsuccessful. No silver adduct could be detected by mass spectrometry. Attempts to measure ${ }^{109} \mathrm{Ag}$ NMR spectra were unsuccessful; the sensitivity of the ${ }^{109} \mathrm{Ag}$ nucleus in $\mathrm{CD}_{3} \mathrm{CN}$ solution is very low and longitudinal relaxation times $\left(\mathrm{T}_{1}\right)$ were too long to run reasonable experiments even when relaxation agents were added. We cannot rule out the possibility that $\mathrm{Ag}^{+}$oxidizes $\mathrm{Ru}^{2+}$ to $\mathrm{Ru}^{3+}$ and that the changes in the ${ }^{1} \mathrm{H}$ NMR spectra arise from the presence of the paramagnetic $\mathrm{Ru}^{3+}$ ion $\left(d^{5}\right)$. However, addition of $\mathrm{Br}_{2}$ to $\left[\mathrm{Ru}(\mathrm{bpy})_{2}(3)\right]\left[\mathrm{PF}_{6}\right]$ results in the appearance of signals between the limits of $\partial+23$ and -34 ppm which is consistent with data reported for polypyridyl $\mu$-oxo complexes of ruthenium(III), ${ }^{43}$ and does not show selective broadening and shifting of signals for the 2-phenylpyridine ligand protons.

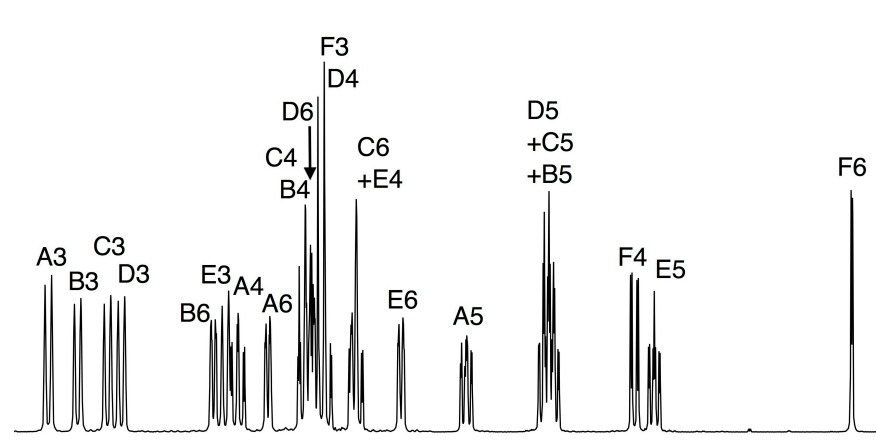

\begin{tabular}{llllllllllllllllllllll}
\hline 8.5 & 8.4 & 8.3 & 8.2 & 8.1 & 8.0 & 7.9 & 7.8 & 7.7 & 7.6 & 7.5 & 7.4 & 7.3 & 7.2 & 7.1 & 7.0 & 6.9 & 6.8 & 6.7 & 6.6 & 6.5 & 6.4
\end{tabular} Fig. 1 Aromatic region of the $500 \mathrm{MHz}{ }^{1} \mathrm{H}$ NMR spectrum of $\left[R u(b p y)_{2}(5)\right]\left[\mathrm{PF}_{6}\right]$ $\left(\mathrm{CD}_{3} \mathrm{CN}, 295 \mathrm{~K}\right)$. See Scheme 5 for the atom labelling scheme. Chemical shifts in $\partial$ $/ \mathrm{ppm}$.

Initial spectrum after workup

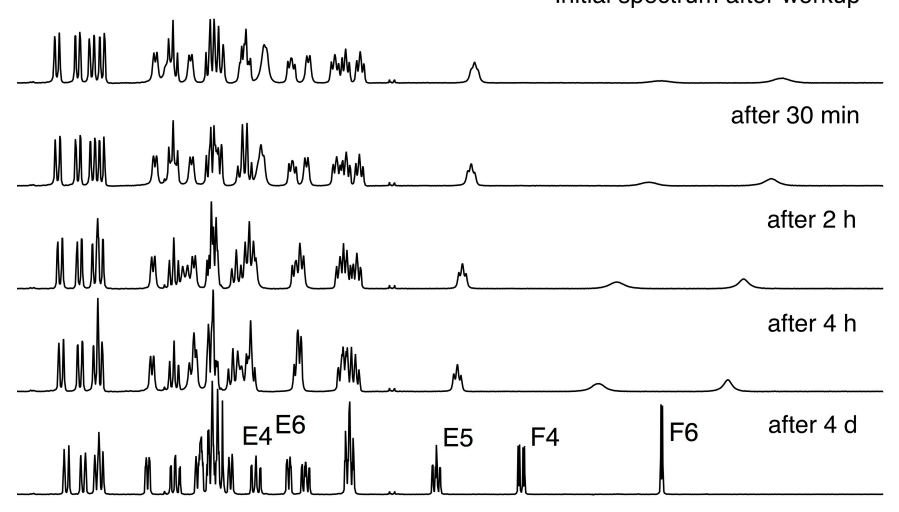

$\begin{array}{lllllllllllllllllllllll}8.5 & 8.3 & 8.1 & 7.9 & 7.7 & 7.5 & 7.3 & 7.1 & 6.9 & 6.7 & 6.5 & 6.3 & 6.1 & 5.9 & 5.7 & 5.5 & 5.3 & 5.1 & 4.1\end{array}$ Fig. 2. Time dependence of the $400 \mathrm{MHz}^{1} \mathrm{H}$ NMR spectrum (aromatic region) of $\left[\mathrm{Ru}(\mathrm{bpy})_{2}(3)\right]\left[\mathrm{PF}_{6}\right]$ in $\mathrm{CD}_{3} \mathrm{CN}(295 \mathrm{~K})$. Chemical shifts in $\partial / \mathrm{ppm}$.

8 | J. Name., 2012, 00, 1-3

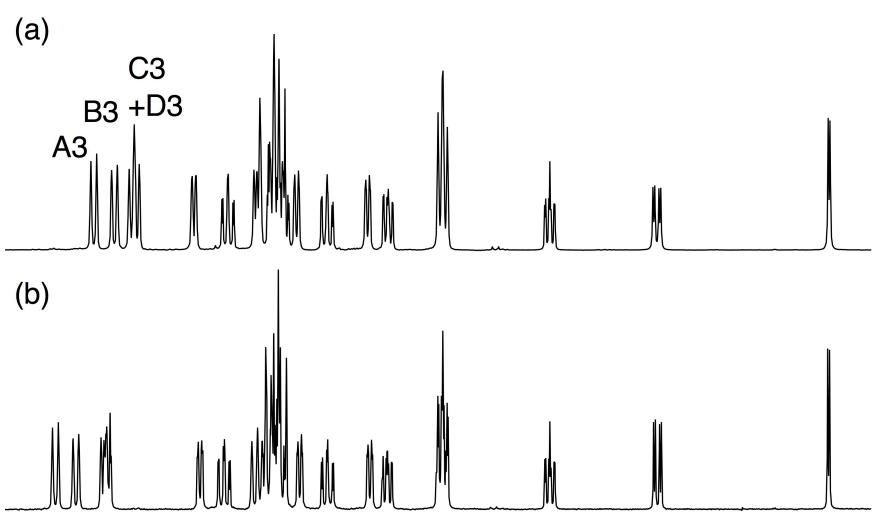

$\begin{array}{lllllllllllllll}8.6 & 8.4 & 8.2 & 8.0 & 7.8 & 7.6 & 7.4 & 7.2 & 7.0 & 6.8 & 6.6 & 6.4 & 6.2 & 6.0 & 5.8\end{array}$ Fig. 3. Room temperature $400 \mathrm{MHz}{ }^{1} \mathrm{H}$ NMR spectrum (aromatic region) of a $\mathrm{CD}_{3} \mathrm{CN}$ solution of (a) $\left[\mathrm{Ru}(\mathrm{bpy})_{2}(3)\right]\left[\mathrm{PF}_{6}\right]$ and $(\mathrm{b})$ after addition of an excess of $\left[{ }^{n} \mathrm{Bu}_{4} \mathrm{~N}\right] \mathrm{Cl}$. Chemical shifts in $\partial / \mathrm{ppm}$. See Scheme 5 for atom labelling.

We note that in order to ensure sharp signals in the ${ }^{1} \mathrm{H}$ NMR spectrum of the $\left[\mathrm{Ru}(\mathrm{bpy})_{2}\left(\mathrm{C}^{\wedge} \mathrm{N}\right)\right]\left[\mathrm{PF}_{6}\right]$ complexes, it is essential either to recrystallize the complex or to dissolve the complex in $\mathrm{MeCN}$ and leave the solution to stand for at least a week; filtration through Celite separates precipitated silver(I) salts.

Rather surprisingly, the number of structurally characterized $\left[\mathrm{Ru}\left(\mathrm{N}^{\wedge} \mathrm{N}\right)_{2}\left(\mathrm{C}^{\wedge} \mathrm{N}\right)\right]^{+}$complexes $\left(\mathrm{N}^{\wedge} \mathrm{N}=\right.$ bpy or phen-based chelate, $\mathrm{C}^{\wedge} \mathrm{N}=$ ppy or derivative of ppy) is limited to only 19 entries in the Cambridge Structural Database ${ }^{44}$ (v. 5.35 with November 2013, February and May 2014 updates, searched using Conquest v. $\left.1.16^{32}\right)$. Single crystals of $\left[\mathrm{Ru}(\mathrm{bpy})_{2}(\mathbf{4})\right]\left[\mathrm{PF}_{6}\right]^{\cdot} \mathrm{MeCN}$ and $\left[\mathrm{Ru}(\mathrm{bpy})_{2}(\mathbf{5})\right]\left[\mathrm{PF}_{6}\right]$ were grown by diffusion of $\mathrm{Et}_{2} \mathrm{O}$ into $\mathrm{MeCN}$ solutions of the complexes. The chiral tris-chelates crystallize in the centrosymmetric $P-1$ space group with both enantiomers of the octahedral cation present in the unit cell. The structures of $\Delta-\left[\mathrm{Ru}(\mathrm{bpy})_{2}(\mathbf{4})\right]^{+}$and $\Delta$-[Ru(bpy $\left.)_{2}(5)\right]^{+}$are shown in Figs. 4 and 5 and important bond distances are listed in the captions. The bond parameters within each ruthenium(II) coordination sphere are similar to those in $\left[\mathrm{Ru}(\mathrm{bpy})_{2}(\mathrm{ppy})\right]\left[\mathrm{PF}_{6}\right] .^{45,46}$ One of the disordered solvent molecules in $\left[\mathrm{Ru}(\mathrm{bpy})_{2}(\mathbf{4})\right]\left[\mathrm{PF}_{6}\right]^{\cdot} \mathrm{MeCN}$ was modelled over two sites of occupancies 68 and $32 \%$. The second could not be modelled in a satisfactory way and SQUEEZE ${ }^{34}$ was therefore used to treat the data. The $\left[\mathrm{PF}_{6}\right]^{-}$anions in both structures are ordered.

The cations in $\left[\mathrm{Ru}(\mathrm{bpy})_{2}(4)\right]\left[\mathrm{PF}_{6}\right] \cdot \mathrm{MeCN}$ are aligned along the $a$-axis with the methyl group (C33) of the ester functionality sandwiched between pyridine rings containing $\mathrm{N} 4^{\mathrm{i}}$ and $\mathrm{N} 5^{\mathrm{i}}$ (Fig. 6a, symmetry code $\mathrm{i}=1+x, y, z$ ). The C33...centroid distances are 3.41 and $4.07 \AA$. This packing interaction is augmented by face-to-face (rings with $\mathrm{N} 1$ and $\mathrm{N} 1{ }^{\mathrm{ii}}$, symmetry code ii $=1-x, 2-y,-z$ ) and edge-to-face (rings with $\mathrm{N} 1$ and $\mathrm{C} 31^{\mathrm{ii}}$ ) contacts between enantiomeric pairs of cations (Fig. 6b). For the face-to-face contact, the separation of the ring planes is $3.51 \AA$ and inter-centroid separation is $3.84 \AA$. The packing in $\left[\mathrm{Ru}(\mathrm{bpy})_{2}(\mathbf{5})\right]\left[\mathrm{PF}_{6}\right]$ features an analogous embrace between enantiomers with the distance between the ring planes (rings 
with $\mathrm{N} 5$ and $\mathrm{N} 5^{\mathrm{i}}$, symmetry code $\left.\mathrm{i}=-x, 2-y, 1-z\right)$ and intercentroid separation being $3.47 \AA$ and $3.83 \AA$ for the face-to-face $\pi$-stacking interaction. The $\left[\operatorname{Ru}(\mathrm{bpy})_{2}(\mathbf{5})\right]^{+}$cations also engage in a second embrace involving pyridine rings containing N4 and $\mathrm{N}^{\mathrm{ii}}$ (symmetry code $\mathrm{ii}=1-x, 2-y,-z$ ), but as Fig. 6c shows, slippage of the rings caused by steric demands of the ${ }^{t} \mathrm{Bu}$ groups results in the $\pi-\pi$ stacking interaction being inefficient (inter-plane and inter-centroid distances $=3.14$ and $4.60 \AA$ A, respectively).

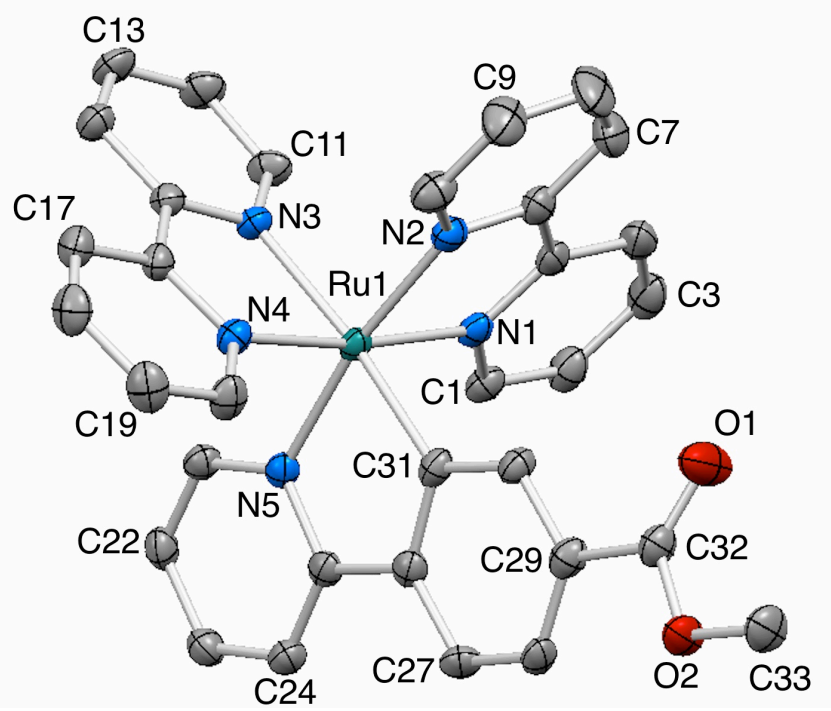

Fig. 4. Structure of the $\Delta$-[Ru(bpy $\left.)_{2}(4)\right]^{+}$cation in $\left[R u(b p y)_{2}(4)\right]\left[\mathrm{PF}_{6}\right]^{\cdot} \mathrm{MeCN}$ with ellipsoids plotted at $40 \%$ probability level and $\mathrm{H}$ atoms omitted for clarity. Selected bond parameters: Ru1-N1 $=2.050(2)$, Ru1-N2 $=2.035(3)$, Ru1-N3 = $2.140(2), \mathrm{Ru}-\mathrm{N} 4=2.066(3), \mathrm{Ru} 1-\mathrm{N} 5=2.068(3), \mathrm{Ru} 1-\mathrm{C} 31=2.025(3), \mathrm{C} 29-\mathrm{C} 32=$ 1.485(4), O1-C32 = 1.207(4), O2-C32 = 1.325(4), O2-C33 = 1.450(4) ̊̊; N1-Ru1$\mathrm{N} 2=78.89(11), \mathrm{N} 3-\mathrm{Ru} 1-\mathrm{N} 4=77.26(10), \mathrm{N} 5-\mathrm{Ru} 1-\mathrm{C} 31=79.63(11), \mathrm{O} 2-\mathrm{C} 32-\mathrm{O} 1=$ $122.0(3)^{\circ}$.

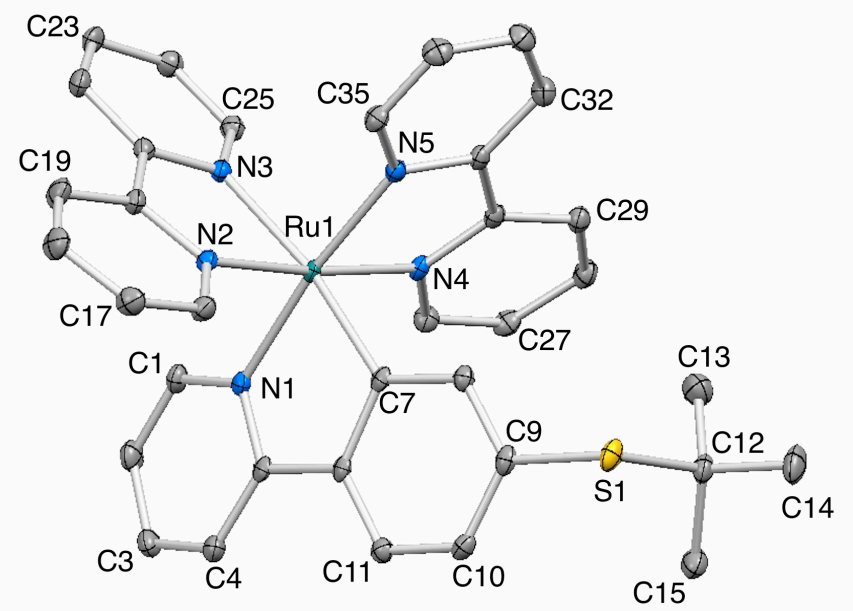

Fig. 5. Structure of the $\Delta$-[Ru(bpy $\left.)_{2}(\mathbf{5})\right]^{+}$cation in $\left[\mathrm{Ru}(\mathrm{bpy})_{2}(\mathbf{5})\right]\left[\mathrm{PF}_{6}\right]$ with ellipsoids plotted at $40 \%$ probability level and $\mathrm{H}$ atoms omitted for clarity. Selected bond distances and angles: Ru1-N5 $=2.0464(16)$, Ru1-N4 $=2.0490(15)$, Ru1-N2 = 2.0696(15), Ru1-N1 = 2.0821(16), Ru1-N3 = 2.1282(15), Ru1-C7 = 2.0326(18), $\mathrm{C} 9-\mathrm{S} 1=1.7865(19), \mathrm{S} 1-\mathrm{C} 12=1.864(2) \AA \AA$ N2-Ru1-N3 = 77.32(6), N5-Ru1-N4 = 78.92(6), C7-Ru1-N1 = 79.75(7), C9-S1-C12 = 102.95(9) ${ }^{\circ}$.

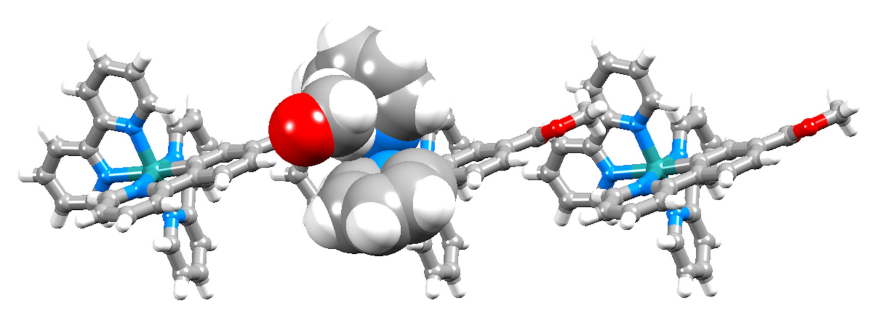

(a)
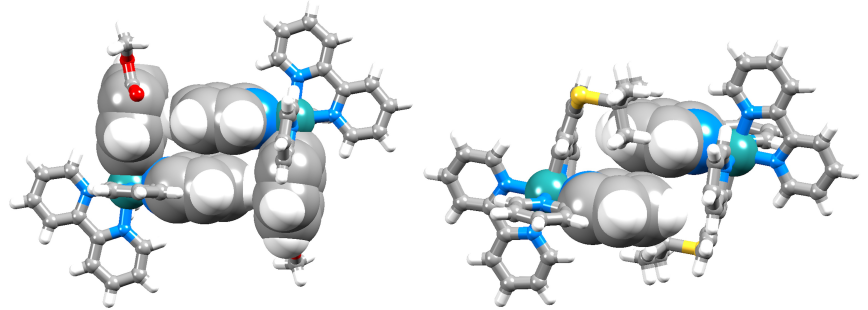

(b)

(c)

Fig. 6. (a) Packing of $\left[\mathrm{Ru}(\mathrm{bpy})_{2}(4)\right]^{+}$cations into chains along the $a$-axis. (b) $\pi-\pi$ and $\mathrm{CH}-\pi$ embrace between enantiomers in $\left[\mathrm{Ru}(\mathrm{bpy})_{2}(4)\right]\left[\mathrm{PF}_{6}\right] \cdot \mathrm{MeCN}$, and (c) slipped $\pi$-stacking interaction between enantiomers in $\left[\mathrm{Ru}(\mathrm{bpy})_{2}(\mathbf{5})\right]\left[\mathrm{PF}_{6}\right]$.

\section{$\left[R u(b p y)_{2}\left(C^{\wedge} N\right)\right]\left[P_{6}\right]$ with $C^{\wedge} N=1-6$ : electronic spectroscopy and electrochemistry}

Fig. 7 compares the solution absorption spectra of $\left[\mathrm{Ru}(\mathrm{bpy})_{2}\left(\mathrm{C}^{\wedge} \mathrm{N}\right)\right]\left[\mathrm{PF}_{6}\right] \quad\left(\mathrm{C}^{\wedge} \mathrm{N}=\mathbf{1 - 6}\right)$ with that of $\left[\mathrm{Ru}(\mathrm{bpy})_{2}(\mathrm{ppy})\right]\left[\mathrm{PF}_{6}\right]$. All complexes exhibit similar spectra with a broad spectral response, consistent with those of related $\left[\mathrm{Ru}(\mathrm{bpy})_{2}\left(\mathrm{C}^{\wedge} \mathrm{N}\right)\right]^{+}$complexes. ${ }^{2,8,14,47}$ The intense bands in the UV region around 250 and $300 \mathrm{~nm}$ arise from spin-allowed ligand-centred $\pi^{*} \leftarrow \pi$ transitions. The two, broad lower energy absorptions in the ranges $350-430 \mathrm{~nm}$ and $430-630 \mathrm{~nm}$ are typical of $\left[\mathrm{Ru}(\mathrm{bpy})_{2}\left(\mathrm{C}^{\wedge} \mathrm{N}\right)\right]^{+}$cations and have previously been assigned to MLCT bands arising from metal-to- $\mathrm{C}^{\wedge} \mathrm{N}$ and metalto-bpy transitions, respectively. ${ }^{8,14,47}$ Consistent with this, Fig. 7 and Table 1 show that the lowest energy bands are largely unaffected by the introduction of substituents into the cyclometallating ligand. Only the spectrum of $\left[\mathrm{Ru}(\mathrm{bpy})_{2}(\mathbf{6})\right]\left[\mathrm{PF}_{6}\right]$, which contains the electron-withdrawing $\mathrm{SO}_{2} \mathrm{Me}$ substituent, shows a noticeable difference (Fig. 7 and Table 1). The $350-430 \mathrm{~nm}$ absorption is most significantly influenced by the introduction of $\mathrm{CO}_{2} \mathrm{Me}$ or $\mathrm{SO}_{2} \mathrm{Me}$ groups (ligands 4 and 6, Fig. 7) and to a lesser extent by the presence of the sulfanyl group in $\mathbf{5}$. 


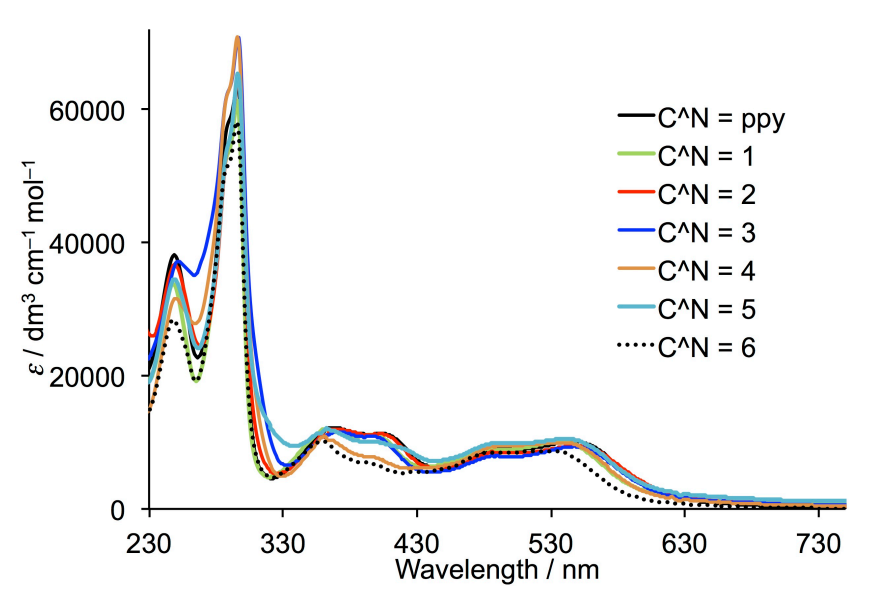

Fig. 7. Solution absorption spectra of $\left[R u(b p y)_{2}\left(C^{\wedge} N\right)\right]\left[P F_{6}\right]$ with $C^{\wedge} N=p p y$ and 1-6 (MeCN, $1 \times 10^{-5} \mathrm{~mol} \mathrm{dm}^{-3}$ ).

The room temperature photoluminescence spectra of $\mathrm{MeCN}$ solutions of the complexes show a dependence on the $\mathrm{C}^{\wedge} \mathrm{N}$ substituent (Fig. 8 and Table 1). Broad, unstructured bands are observed. As previously reported for related complexes, ${ }^{8,11,47}$ $\left[\mathrm{Ru}(\mathrm{bpy})_{2}\left(\mathrm{C}^{\wedge} \mathrm{N}\right)\right]\left[\mathrm{PF}_{6}\right]$ with $\mathrm{C}^{\wedge} \mathrm{N}=\mathbf{1 - 6}$ are very weak emitters in solution at room temperature and exhibit quantum yields of $<1 \%$ (the limit of detection of our instrument). This is consistent with work of Castellano and coworkers who were unable to obtain reliable PLQY values for $\left[\mathrm{Ru}(\mathrm{bpy})_{2}(\mathrm{ppy})\right]\left[\mathrm{PF}_{6}\right]$ and related complexes due to their extremely low values. ${ }^{47}$ Thus, we focus only on the trends in the emission maxima. Using $\left[\mathrm{Ru}(\mathrm{bpy})_{2}(\mathrm{ppy})\right]\left[\mathrm{PF}_{6}\right]$ as a reference point, the introduction of 4-methyl or 4-methoxy substituents into the phenyl ring of the $\mathrm{C}^{\wedge} \mathrm{N}$ ligand has essentially no effect on $\lambda_{\text {em }}{ }^{\max }$ (Table 1). A blue shift is observed when electronwithdrawing substituents are introduced, consistent with a lowering of the energy of the HOMO. For the most electronwithdrawing group $\left(\mathrm{SO}_{2} \mathrm{Me}\right)$, a blue-shift of $43 \mathrm{~nm}$ occurs on going from $\left[\mathrm{Ru}(\mathrm{bpy})_{2}(\mathrm{ppy})\right]\left[\mathrm{PF}_{6}\right]$ to $\left[\mathrm{Ru}(\mathrm{bpy})_{2}(6)\right]\left[\mathrm{PF}_{6}\right]$.

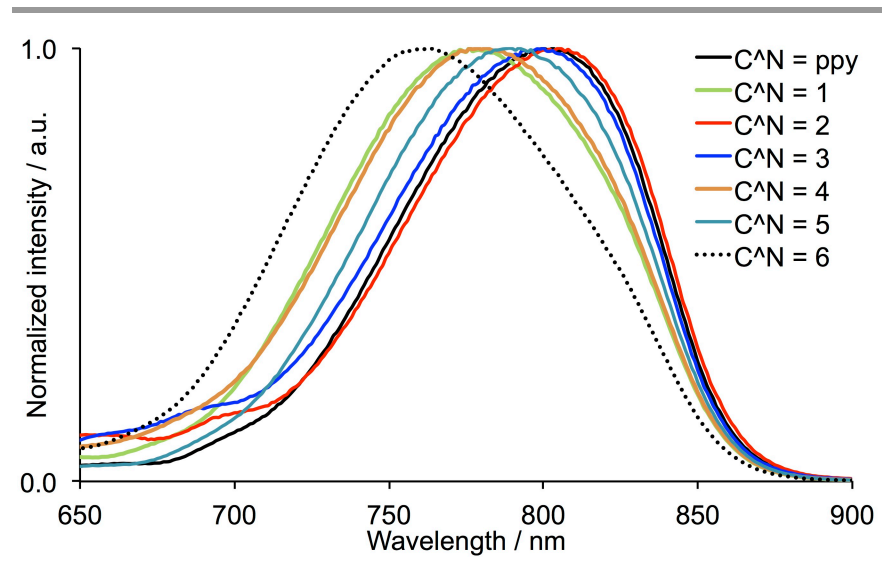

Fig. 8. Normalized solution emission spectra of $\left[R u(b p y)_{2}\left(C^{\wedge} N\right)\right]\left[P F_{6}\right]$ with $C^{\wedge} N=$ ppy and 1-6 (MeCN, $5 \times 10^{-5} \mathrm{~mol} \mathrm{dm}^{-3}$ ).
Table 1. MLCT-absorption ${ }^{\mathrm{a}}$ and emission ${ }^{\mathrm{b}}$ maxima for $\left[\mathrm{Ru}(\mathrm{bpy})_{2}\left(\mathrm{C}^{\wedge} \mathrm{N}\right)\right]\left[\mathrm{PF}_{6}\right]$ with $\mathrm{C}^{\wedge} \mathrm{N}=$ ppy and 1-6 (MeCN solutions).

\begin{tabular}{|c|c|c|c|c|}
\hline \multirow[t]{2}{*}{ Complex } & \multicolumn{2}{|c|}{ MLCT $\lambda_{\max } / \mathrm{nm}$} & \multirow{2}{*}{$\begin{array}{c}\lambda_{\text {exc }} / \\
\mathrm{nm}\end{array}$} & \multirow{2}{*}{$\begin{array}{c}\lambda_{\mathrm{em}}^{\max } \\
\mathrm{nm} \\
\end{array}$} \\
\hline & Ru-to- $\mathrm{C}^{\wedge} \mathrm{N}$ & Ru-to-bpy & & \\
\hline$\left[\mathrm{Ru}(\mathrm{bpy})_{2}(\mathrm{ppy})\right]\left[\mathrm{PF}_{6}\right]$ & 369,403 & 488,546 & 575 & 803 \\
\hline$\left[\mathrm{Ru}(\mathrm{bpy})_{2}(\mathbf{1})\right]\left[\mathrm{PF}_{6}\right]$ & 368,399 & 485,538 & 560 & 776 \\
\hline$\left[\mathrm{Ru}(\mathrm{bpy})_{2}(\mathbf{2})\right]\left[\mathrm{PF}_{6}\right]$ & 372,398 & 486,547 & 575 & 805 \\
\hline$\left[\mathrm{Ru}(\mathrm{bpy})_{2}(\mathbf{3})\right]\left[\mathrm{PF}_{6}\right]$ & 360,395 & 485,538 & 570 & 800 \\
\hline$\left[\mathrm{Ru}(\mathrm{bpy})_{2}(4)\right]\left[\mathrm{PF}_{6}\right]$ & 362,395 & 492,541 & 565 & 781 \\
\hline$\left[\mathrm{Ru}(\mathrm{bpy})_{2}(\mathbf{5})\right]\left[\mathrm{PF}_{6}\right]$ & 362,395 & 492,541 & 570 & 792 \\
\hline$\left[\mathrm{Ru}(\mathrm{bpy})_{2}(\mathbf{6})\right]\left[\mathrm{PF}_{6}\right]$ & 358,393 & 485,530 & 555 & 760 \\
\hline
\end{tabular}

${ }^{a}$ Extinction coefficients for the MLCT bands are given in the experimental section. ${ }^{\mathrm{b}}$ Degassed solutions.

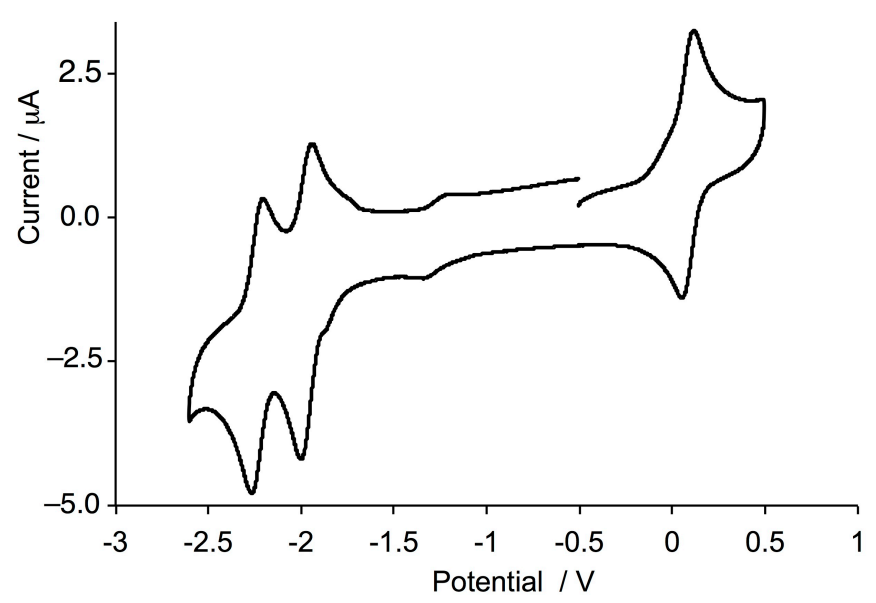

Fig. 9. Cyclic voltammogram of $\left[\mathrm{Ru}(\mathrm{bpy})_{2}(3)\right]\left[\mathrm{PF}_{6}\right]$ (degassed MeCN solution) with respect to $\mathrm{Fc} / \mathrm{Fc}^{+}$; scan rate $=0.1 \mathrm{~V} \mathrm{~s}^{-1}$.

The electrochemical data of the ruthenium(II) complexes are summarized in Table 2, and a representative cyclic voltammogram (CV) is shown in Fig. 9. Each complex undergoes a reversible metal-centred oxidation. The reversible $\mathrm{Ru}^{3+} / \mathrm{Ru}^{2+}$ process for $\left[\mathrm{Ru}(\mathrm{bpy})_{2}(\mathrm{ppy})\right]\left[\mathrm{PF}_{6}\right]$ occurs at $+0.09 \mathrm{~V}$ versus $\mathrm{Fc}^{+} / \mathrm{Fc}$; literature data are with respect to NHE or SCE. ${ }^{1,2,8}$ Introduction of electron-withdrawing substituents on going to $\left[\mathrm{Ru}(\mathrm{bpy})_{2}(\mathbf{1})\right]\left[\mathrm{PF}_{6}\right], \quad\left[\mathrm{Ru}(\mathrm{bpy})_{2}(\mathbf{4})\right]\left[\mathrm{PF}_{6}\right]$, $\left[\mathrm{Ru}(\mathrm{bpy})_{2}(\mathbf{5})\right]\left[\mathrm{PF}_{6}\right]$ and $\left[\mathrm{Ru}(\mathrm{bpy})_{2}(\mathbf{6})\right]\left[\mathrm{PF}_{6}\right]$ shifts the $\mathrm{Ru}^{3+} / \mathrm{Ru}^{2+}$ couple to higher potential in the order (for the substituents) $\mathrm{SO}_{2} \mathrm{Me}>\mathrm{CO}_{2} \mathrm{Me}>\mathrm{F}>{ }^{t} \mathrm{BuS}$. Introduction of the electrondonating $\mathrm{Me}$ or $\mathrm{OMe}$ groups in $\left[\mathrm{Ru}(\mathrm{bpy})_{2}(\mathbf{2})\right]\left[\mathrm{PF}_{6}\right]$ and $\left[\mathrm{Ru}(\mathrm{bpy})_{2}(\mathbf{3})\right]\left[\mathrm{PF}_{6}\right]$, respectively, causes small shifts to lower potential. The trend in potentials for the oxidation process is consistent with the HOMO being centred on the metal and cyclometallating ligand. Each complex exhibits two, ligandbased reversible reduction processes. The invariance of the potential for a given process (first or second reduction) is consistent with localization of the LUMO on the bpy ligands. For $\left[\mathrm{Ru}(\mathrm{bpy})_{2}(6)\right]\left[\mathrm{PF}_{6}\right]$, a third reversible reduction is observed. On going from $\left[\mathrm{Ru}(\mathrm{bpy})_{3}\right]^{2+}$ to $\left[\mathrm{Ru}(\mathrm{bpy})_{2}(\mathrm{ppy})\right]^{+}$, the ligandbased reduction processes shift to lower potential as a result of greater $\pi$-back-bonding from the ruthenium(II) centre. ${ }^{8}$ The introduction of the strongly electron-withdrawing $\mathrm{SO}_{2} \mathrm{Me}$ group in $\left[\mathrm{Ru}(\mathrm{bpy})_{2}(\mathbf{6})\right]^{+}$opposes the latter, and this is consistent with 
the appearance of a third reduction process. Note that a third process is present within the solvent accessible window in $\left[\mathrm{Ru}(\mathrm{bpy})_{3}\right]^{2+}$ but not in $\left[\mathrm{Ru}(\mathrm{bpy})_{2} \text { (ppy) }\right]^{+}{ }^{8}$

Table 2. Redox potentials for $\left[\mathrm{Ru}(\mathrm{bpy})_{2}\left(\mathrm{C}^{\wedge} \mathrm{N}\right)\right]\left[\mathrm{PF}_{6}\right]$ with $\mathrm{C}^{\wedge} \mathrm{N}=$ ppy and 1-6 (degassed MeCN solutions) with respect to $\mathrm{Fc} / \mathrm{Fc}^{+} ; 0.1 \mathrm{M}\left[{ }^{\mathrm{n}} \mathrm{Bu}_{4} \mathrm{~N}\right]\left[\mathrm{PF}_{6}\right]$ as supporting electrolyte and a scan rate of $0.1 \mathrm{~V} \mathrm{~s}^{-1}$.

\begin{tabular}{|c|c|c|c|c|c|}
\hline Complex & $\begin{array}{c}E_{1 / 2}{ }^{\mathrm{ox}} / \\
\mathrm{V}\end{array}$ & $\begin{array}{c}E_{1 / 2}{ }^{\text {redl }} \\
/ \mathrm{V}\end{array}$ & $\begin{array}{c}E_{1 / 2}{ }^{\text {red2 }} \\
/ \mathrm{V}\end{array}$ & $\begin{array}{c}E_{1 / 2}{ }^{\text {red3 }} \\
/ \mathrm{V}\end{array}$ & $\begin{array}{c}\Delta E_{1 / 2} / \\
\mathrm{V}\end{array}$ \\
\hline$\left[\mathrm{Ru}(\mathrm{bpy})_{2}(\mathrm{ppy})\right]\left[\mathrm{PF}_{6}\right]$ & +0.09 & -1.96 & -2.22 & & 2.05 \\
\hline$\left[\mathrm{Ru}(\mathrm{bpy})_{2}(\mathbf{1})\right]\left[\mathrm{PF}_{6}\right]$ & +0.15 & -1.97 & -2.22 & & 2.12 \\
\hline$\left[\mathrm{Ru}(\mathrm{bpy})_{2}(\mathbf{2})\right]\left[\mathrm{PF}_{6}\right]$ & +0.06 & -1.98 & -2.24 & & 2.04 \\
\hline$\left[\mathrm{Ru}(\mathrm{bpy})_{2}(\mathbf{3})\right]\left[\mathrm{PF}_{6}\right]$ & +0.08 & -1.98 & -2.25 & & 2.06 \\
\hline$\left[\mathrm{Ru}(\mathrm{bpy})_{2}(\mathbf{4})\right]\left[\mathrm{PF}_{6}\right]$ & +0.17 & -1.94 & -2.19 & & 2.11 \\
\hline$\left[\mathrm{Ru}(\mathrm{bpy})_{2}(\mathbf{5})\right]\left[\mathrm{PF}_{6}\right]$ & +0.11 & -1.98 & -2.24 & & 2.09 \\
\hline$\left[\mathrm{Ru}(\mathrm{bpy})_{2}(\mathbf{6})\right]\left[\mathrm{PF}_{6}\right]$ & +0.21 & -1.95 & -2.19 & -2.43 & 2.16 \\
\hline
\end{tabular}

\section{$\left[\operatorname{Ru}(\mathrm{bpy})_{2}\left(\mathrm{C}^{\wedge} \mathrm{N}\right)\right]\left[\mathrm{PF}_{6}\right]$ with $\mathrm{C}^{\wedge} \mathrm{N}=7$ and 9, and $\left[\mathrm{Ru}(\mathrm{bpy})_{2}(8)\right]$}

The compounds $\left[\mathrm{Ru}(\mathrm{bpy})_{2}\left(\mathrm{C}^{\wedge} \mathrm{N}\right)\right]\left[\mathrm{PF}_{6}\right]$ with $\mathrm{C}^{\wedge} \mathrm{N}=\mathbf{7}$ and 9 were synthesized as described for the analogues with $\mathrm{C}^{\wedge} \mathrm{N}=\mathbf{1}$ 6. The synthesis of $\left[\mathrm{Ru}(\mathrm{bpy})_{2}(\mathbf{8})\right]$ employed a method described by Bomben et al. ${ }^{11}$ for hydrolysis of a pyridine-attached ester substituent; heating $\left[\mathrm{Ru}(\mathrm{bpy})_{2}(7)\right]\left[\mathrm{PF}_{6}\right]$ in a mixture of DMF, $\mathrm{H}_{2} \mathrm{O}$ and $\mathrm{NEt}_{3}$ at $100{ }^{\circ} \mathrm{C}$ for 16 hours yielded the zwitter-ion $\left[\mathrm{Ru}(\mathrm{bpy})_{2}(\mathbf{8})\right]$ in $89 \%$ yield. For each of $\left[\mathrm{Ru}(\mathrm{bpy})_{2}\left(\mathrm{C}^{\wedge} \mathrm{N}\right)\right]\left[\mathrm{PF}_{6}\right]$ $\left(\mathrm{C}^{\wedge} \mathrm{N}=7\right.$ and 9), the base peak in the ESI mass spectrum arose from the $\left[\mathrm{M}-\mathrm{PF}_{6}\right]^{+}$ion and the isotope pattern matched that calculated. The formation of a neutral compound $\left[\mathrm{Ru}(\mathrm{bpy})_{2}(\mathbf{8})\right]$ with ligand $\mathrm{H}_{2} \mathbf{8}$ was supported by high resolution mass spectrometry and by the absence of signals for $\left[\mathrm{PF}_{6}\right]^{-}$in the ${ }^{31} \mathrm{P}$ and ${ }^{19} \mathrm{~F}$ NMR spectra. The MALDI-TOF mass spectrum of $\left[\mathrm{Ru}(\mathrm{bpy})_{2}(\mathbf{8})\right]$ showed a peak envelope at $\mathrm{m} / \mathrm{z} 612.3$ consistent with $[\mathrm{M}+\mathrm{H}]^{+}$; signals were also observed for $\left[\mathrm{M}+\mathrm{H}-\mathrm{CO}_{2}\right]^{+}$and $[\mathrm{M}+\mathrm{H}-\mathrm{bpy}]^{+}$.

The ${ }^{1} \mathrm{H}$ NMR spectra of $\mathrm{CD}_{3} \mathrm{CN}$ solutions of $\left[\mathrm{Ru}(\mathrm{bpy})_{2}\left(\mathrm{C}^{\wedge} \mathrm{N}\right)\right]\left[\mathrm{PF}_{6}\right]\left(\mathrm{C}^{\wedge} \mathrm{N}=7\right.$ and 9) showed the same broadening of signals observed for the complexes containing 16 (see Fig. 2). Sharp spectra (illustrated for $\left[\mathrm{Ru}(\mathrm{bpy})_{2}(7)\right]\left[\mathrm{PF}_{6}\right]$ in Fig. 10) were obtained once MeCN solutions of the product had been left to stand for several days and then filtered through Celite. In contrast, and consistent with the absence of $\mathrm{Ag}^{+}$ions in the synthesis, the ${ }^{1} \mathrm{H}$ NMR spectrum of a freshly prepared sample of $\left[\mathrm{Ru}(\mathrm{bpy})_{2}(\mathbf{8})\right]$ showed sharp signals which did not shift with time. Berlinguette and coworkers have previously reported $\left[\mathrm{Ru}(\mathrm{bpy})_{2}(\mathrm{H} 8)\right]\left[\mathrm{NO}_{3}\right]$, prepared under basic conditions from reaction of cis-[Ru(bpy) $\left.{ }_{2} \mathrm{Cl}_{2}\right]$ and $\mathrm{H}_{2} \mathbf{8}$ in the presence of $\mathrm{AgNO}_{3} .{ }^{8} \mathrm{We}$ note that the spectroscopic properties reported for $\left[\mathrm{Ru}(\mathrm{bpy})_{2}(\mathrm{H8})\right]\left[\mathrm{NO}_{3}\right] \quad(\mathrm{NMR}$ spectra recorded in $\left.\mathrm{CD}_{3} \mathrm{OD} / \mathrm{NaOD}\right)^{8}$ are very similar to those we observe (in $\mathrm{CD}_{3} \mathrm{OD}$ or in $\mathrm{CD}_{3} \mathrm{CN}$ ) for $\left[\mathrm{Ru}(\mathrm{bpy})_{2}(\mathbf{8})\right]$.

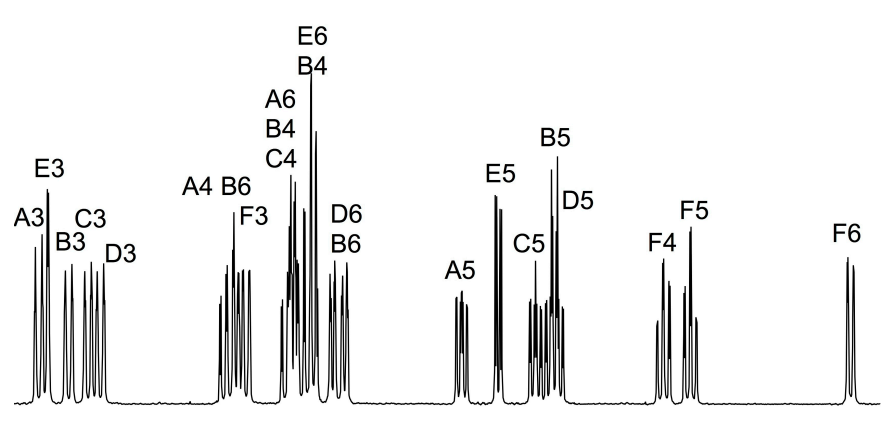

$\begin{array}{lllllllllllllllllllllll}3.5 & 8.4 & 8.3 & 8.2 & 8.1 & 8.0 & 7.9 & 7.8 & 7.7 & 7.6 & 7.5 & 7.4 & 7.3 & 7.2 & 7.1 & 7.0 & 6.9 & 6.8 & 6.7 & 6.6 & 6.5 & 6\end{array}$ Fig. 10. Room temperature $500 \mathrm{MHz}{ }^{1} \mathrm{H}$ NMR spectrum (aromatic region) of a $\mathrm{CD}_{3} \mathrm{CN}$ solution of $\left[\mathrm{Ru}(\mathrm{bpy})_{2}(7)\right]\left[\mathrm{PF}_{6}\right]$. Chemical shifts in $\partial / \mathrm{ppm}$. See Scheme 5 for atom labelling.

Solution ${ }^{1} \mathrm{H}$ and ${ }^{13} \mathrm{C}$ NMR spectra of the three complexes were assigned by $2 \mathrm{D}$-methods. In the NOESY spectrum, crosspeaks between signals for $\mathrm{H}^{\mathrm{B} 6}, \mathrm{H}^{\mathrm{C} 6}$ and $\mathrm{H}^{\mathrm{F} 6}$ and between those for $\mathrm{H}^{\mathrm{A} 6}, \mathrm{H}^{\mathrm{D} 6}$, and $\mathrm{H}^{\mathrm{E} 6}$ assisted in confirmation of ring assignment. For each complex, the doublet at lowest frequency (Fig. 10) was assigned to $\mathrm{H}^{\mathrm{F} 6}$ of the cyclometallated ring. For $\left[\mathrm{Ru}(\mathrm{bpy})_{2}(\mathbf{9})\right]\left[\mathrm{PF}_{6}\right]$, additional signals in the aromatic region were observed consistent with the presence of the phenyl spacer (ring G). For $\left[\mathrm{Ru}(\mathrm{bpy})_{2}(7)\right]\left[\mathrm{PF}_{6}\right]$, the methyl ester gives rise to singlets at 3.91 and $53.4 \mathrm{ppm}$ in the ${ }^{1} \mathrm{H}$ and ${ }^{13} \mathrm{C}$ NMR spectra, respectively. The phosphonate ester group in $\left[\mathrm{Ru}(\mathrm{bpy})_{2}(\mathbf{9})\right]\left[\mathrm{PF}_{6}\right]$ gave rise to characteristic multiplets at $\partial$ 4.08 and $1.29 \mathrm{ppm}$, and at $\partial 63.1$ and $16.7 \mathrm{ppm}$ in the ${ }^{1} \mathrm{H}$ and ${ }^{13} \mathrm{C}$ NMR spectra, respectively, and a signal at $\partial 17.0 \mathrm{ppm}$ in the ${ }^{31} \mathrm{P}$ NMR spectrum. The reaction of $c i s-\left[\mathrm{Ru}(\mathrm{bpy})_{2} \mathrm{Cl}_{2}\right]$ with $\mathrm{H} 9$ to give the diester $\left[\mathrm{Ru}(\mathrm{bpy})_{2}(\mathbf{9})\right]\left[\mathrm{PF}_{6}\right]$ as the isolated product contrasts with the tendency of diethyl $2,2^{\prime}: 6^{\prime}, 2^{\prime \prime}-$ terpyridine-4'-phosphonate to undergo partial hydrolysis to the monoester during formation of ruthenium(II) complexes. ${ }^{18,19,21}$

X-Ray quality single crystals of $\left[\mathrm{Ru}(\mathrm{bpy})_{2}(7)\right]\left[\mathrm{PF}_{6}\right]$ were grown by diffusion of $\mathrm{Et}_{2} \mathrm{O}$ into a $\mathrm{CH}_{2} \mathrm{Cl}_{2}$ solution of the compound. The octahedral tris(chelate) $\left[\mathrm{Ru}(\mathrm{bpy})_{2}(7)\right]^{+}$is chiral, and as the complex crystallizes in the centrosymmetric space group $P 2_{1} / n$, both the $\Lambda$ - and $\Delta$-enantiomers are present in the lattice. The structure of the $\Lambda-\left[\operatorname{Ru}(\text { bpy })_{2}(7)\right]^{+}$cation is shown in Fig. $11 \mathrm{a}$; the figure caption gives selected bond parameters. Each chelating ligand deviates little from planarity; the angles between the planes through the rings containing N2/N3, N4/N5 and $\mathrm{C} 1 / \mathrm{N} 1=5.1,9.6$ and $7.2^{\circ}$, respectively. $\Lambda$ - and $\Delta$ $\left[\mathrm{Ru}(\mathrm{bpy})_{2}(7)\right]^{+}$cations interact through efficient $\pi-\pi$ and $\mathrm{CH}-\pi$ contacts (Fig. 11b) to give a packing motif analogous to that in $\left[\mathrm{Ru}(\mathrm{bpy})_{2}(4)\right]\left[\mathrm{PF}_{6}\right]$ (Fig. 6b). For the face-to-face $\pi$-interaction between pyridine rings with $\mathrm{N} 5$ and $\mathrm{N} 5^{\mathrm{i}}$ (symmetry code $\mathrm{i}=1-$ $x, 1-y, 1-z)$, the inter-plane and inter-centroid distances are 3.30 and $3.61 \AA$, respectively. The complementary edge-to-face contact is between the pyridine ring with $\mathrm{N} 5$ and the cyclometallated ring with $\mathrm{C}^{\mathrm{i}}(\mathrm{C} 31 \mathrm{H} 31 \ldots$ centroid $=2.41 \AA)$. The different substitution pattern of the $\mathrm{CO}_{2} \mathrm{Me}$ group in the $\mathrm{C}^{\wedge} \mathrm{N}$ ligand in $\left[\mathrm{Ru}(\mathrm{bpy})_{2}(4)\right]^{+}$and $\left[\mathrm{Ru}(\mathrm{bpy})_{2}(7)\right]^{+}$impacts on the packing interactions involving the ester group. In contrast to the 
accommodation of the $\mathrm{CO}_{2} \mathrm{Me}$ group between pairs of pyridine rings in $\left[\mathrm{Ru}(\mathrm{bpy})_{2}(4)\right]\left[\mathrm{PF}_{6}\right]$ (Fig. 6a), methyl ester groups in $\left[\mathrm{Ru}(\mathrm{bpy})_{2}(7)\right]\left[\mathrm{PF}_{6}\right]$ are organized in head-to-tail pairs although the shortest $\mathrm{C} 12-\mathrm{O} 1 \ldots \mathrm{H} 8 \mathrm{~A}^{\mathrm{ii}}-\mathrm{C} 8^{\mathrm{ii}}$ contacts $(2.94 \AA$, symmetry code $\mathrm{ii}=2-x, 1-y, 2-z)$ are rather long to represent significant hydrogen bond interactions. ${ }^{48}$

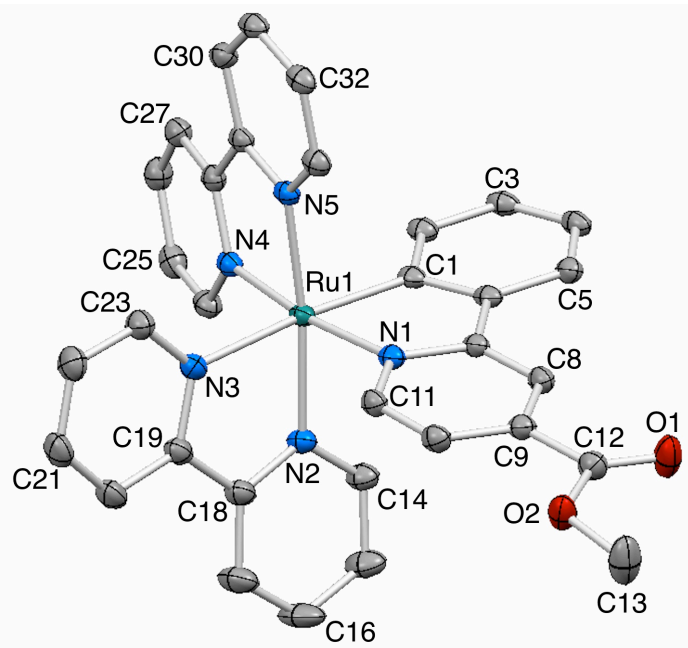

(a)

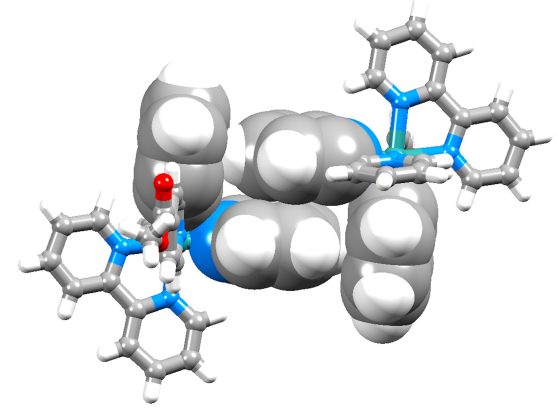

(b)

Fig. 11. (a) Structure of the $\Lambda$-[Ru(bpy $\left.)_{2}(\mathbf{7})\right]^{+}$cation in $\left[R u(b p y)_{2}(7)\right]\left[P_{6}\right]$ with ellipsoids plotted at $40 \%$ probability level and $\mathrm{H}$ atoms omitted for clarity. Selected bond parameters: Ru1-C1 = 2.0347(19), Ru1-N1 = 2.0614(17), Ru1-N2 $=2.0662(16)$, Ru1-N3 = 2.1391(17), Ru1-N4 = 2.0381(16), Ru1-N5 = 2.0399(15), $\mathrm{C} 9-\mathrm{C} 12=1.488(3), \mathrm{C} 12-\mathrm{O} 1=1.205(3), \mathrm{C} 12-\mathrm{O} 2=1.331(3), \mathrm{O} 2-\mathrm{C} 13=1.437(3) \AA$ C1-Ru1-N1 = 79.68(7), N2-Ru1-N3 = 77.59(6), N4-Ru1-N5 = 79.01(6), O1-C12$\mathrm{O} 2=124.3(2), \mathrm{O} 2-\mathrm{C} 12-\mathrm{C} 9=111.14(17), \mathrm{O} 1-\mathrm{C} 12-\mathrm{C} 9=124.54(19), \mathrm{C} 12-\mathrm{O} 2-\mathrm{C} 13$ $=116.01(18)^{\circ}$. (b) Efficient face-to-face and edge-to-face interactions between enantiomers in $\left[\mathrm{Ru}(\mathrm{bpy})_{2}(7)\right]\left[\mathrm{PF}_{6}\right]$.

Fig. 12 shows the solution absorption spectra of $\left[\mathrm{Ru}(\mathrm{bpy})_{2}(7)\right]\left[\mathrm{PF}_{6}\right],\left[\mathrm{Ru}(\mathrm{bpy})_{2}(\mathbf{9})\right]\left[\mathrm{PF}_{6}\right]$ and $\left[\mathrm{Ru}(\mathrm{bpy})_{2}(\mathbf{8})\right]$. The intense, high-energy ligand-based absorptions (below $300 \mathrm{~nm}$ ) are similar to those observed for $\left[\mathrm{Ru}(\mathrm{bpy})_{2}(\mathrm{ppy})\right]\left[\mathrm{PF}_{6}\right]$ and $\left[\mathrm{Ru}(\mathrm{bpy})_{2}\left(\mathrm{C}^{\wedge} \mathrm{N}\right)\right]\left[\mathrm{PF}_{6}\right]\left(\mathrm{C}^{\wedge} \mathrm{N}=\mathbf{1}-\mathbf{6}\right)$. The enhanced intensity of the band at $253 \mathrm{~nm}$ on going from $\left[\mathrm{Ru}(\mathrm{bpy})_{2}(7)\right]\left[\mathrm{PF}_{6}\right]$ and $\left[\mathrm{Ru}(\mathrm{bpy})_{2}(\mathbf{8})\right]$, to $\left[\mathrm{Ru}(\mathrm{bpy})_{2}(\mathbf{9})\right]\left[\mathrm{PF}_{6}\right]$ is consistent with the introduction of the phenyl spacer in the latter complex. As mentioned earlier, the 350-430 $\mathrm{nm}$ and 430-630 nm MLCT bands in $\left[\mathrm{Ru}(\mathrm{bpy})_{2}(\mathrm{ppy})\right]^{+}$arise from Ru-to-ppy and Ru-to-bpy transitions, respectively, ${ }^{8,14,47}$ and for the $\mathrm{C}^{\wedge} \mathrm{N}$ ligands with substituents in the phenyl ring, the spectra in Fig. 7 and Table 1 suggested an analogous partitioning of character. However, Fig. 12 and Table 3 show that when the $C^{\wedge} \mathrm{N}$ ligand is 7 or $\mathbf{9}$, assignments are ambiguous. The introduction of the diethyl phenylenephosphonate group has the greatest influence on the absorption in the region (red line in Fig. 12), and improves the overall spectral response of the complex.

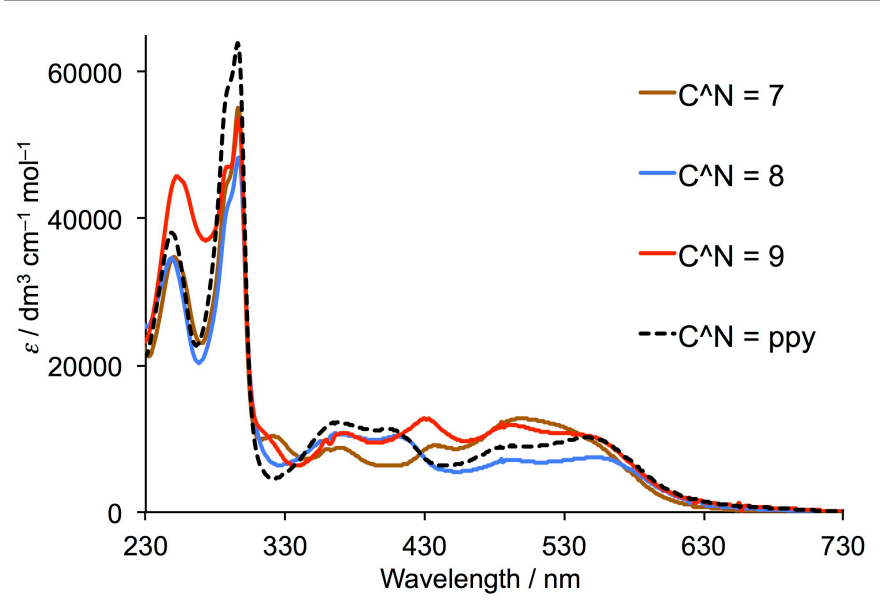

Fig. 12. Solution absorption spectra of $\left[R u(b p y)_{2}(7)\right]\left[P F_{6}\right], \quad\left[R u(b p y)_{2}(9)\right]\left[P F_{6}\right]$ and neutral complex $\left[\mathrm{Ru}(\mathrm{bpy})_{2}(\mathbf{8})\right]$ compared to $\left[\mathrm{Ru}(\mathrm{bpy})_{2}(\mathrm{ppy})\right]\left[\mathrm{PF}_{6}\right]\left(\mathrm{MeCN}, 1 \times 10^{-5}\right.$

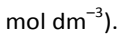

Table 3. MLCT-absorption ${ }^{\mathrm{a}}$ and emission maxima ${ }^{\mathrm{b}}$ for $\left[\mathrm{Ru}(\mathrm{bpy})_{2}(7)\right]\left[\mathrm{PF}_{6}\right]$, $\left[\mathrm{Ru}(\mathrm{bpy})_{2}(\mathbf{9})\right]\left[\mathrm{PF}_{6}\right]$ and neutral complex $\left[\mathrm{Ru}(\mathrm{bpy})_{2}(\mathbf{8})\right]$ compared to $\left[\mathrm{Ru}(\text { bpy })_{2}(\right.$ ppy $\left.)\right]\left[\mathrm{PF}_{6}\right](\mathrm{MeCN}$ solutions).

\begin{tabular}{|c|c|c|c|}
\hline Complex & MLCT $\lambda_{\max } / \mathrm{nm}$ & $\begin{array}{c}\lambda_{\text {exc }} / \\
\mathrm{nm}\end{array}$ & $\begin{array}{c}\lambda_{\mathrm{em}}^{\max } \\
\mathrm{nm}\end{array}$ \\
\hline$\left[\mathrm{Ru}(\mathrm{bpy})_{2}(\mathrm{ppy})\right]\left[\mathrm{PF}_{6}\right]$ & $369,403,488,546$ & 575 & 803 \\
\hline$\left[\mathrm{Ru}(\mathrm{bpy})_{2}(\mathbf{7})\right]\left[\mathrm{PF}_{6}\right]$ & $322,368,438,499$ & 575 & 784 \\
\hline$\left[\mathrm{Ru}(\mathrm{bpy})_{2}(\mathbf{8})\right]$ & $365,408,492,551$ & 580 & 809 \\
\hline$\left[\mathrm{Ru}(\mathrm{bpy})_{2}(\mathbf{1 1})\right]\left[\mathrm{PF}_{6}\right]$ & $371,430,491,537$ & 570 & 798 \\
\hline
\end{tabular}

${ }^{a}$ Extinction coefficients for the MLCT bands are given in the experimental section. ${ }^{\mathrm{b}}$ Degassed solutions.

The compounds $\left[\mathrm{Ru}(\mathrm{bpy})_{2}(\mathbf{8})\right], \quad\left[\mathrm{Ru}(\mathrm{bpy})_{2}(7)\right]\left[\mathrm{PF}_{6}\right]$ and $\left[\mathrm{Ru}(\mathrm{bpy})_{2}(\mathbf{9})\right]\left[\mathrm{PF}_{6}\right]$ are weakly emissive, and emission maxima are given in Table 3. As Fig. 13 shows, the only significant effect compared to $\left[\mathrm{Ru}(\mathrm{bpy})_{2}(\mathrm{ppy})\right]\left[\mathrm{PF}_{6}\right]\left(\lambda_{\max }=803 \mathrm{~nm}\right)$ is caused by the introduction of the electron-withdrawing methyl ester group in $\left[\mathrm{Ru}(\mathrm{bpy})_{2}(7)\right]\left[\mathrm{PF}_{6}\right]\left(\lambda_{\max }=784 \mathrm{~nm}\right)$, consistent with a lowering of the HOMO which is centred on the cyclometallating ligand. No such blue-shift is observed when the phenylene spacer is present between the electronwithdrawing phosphonate group and the phenylpyridine domain. 


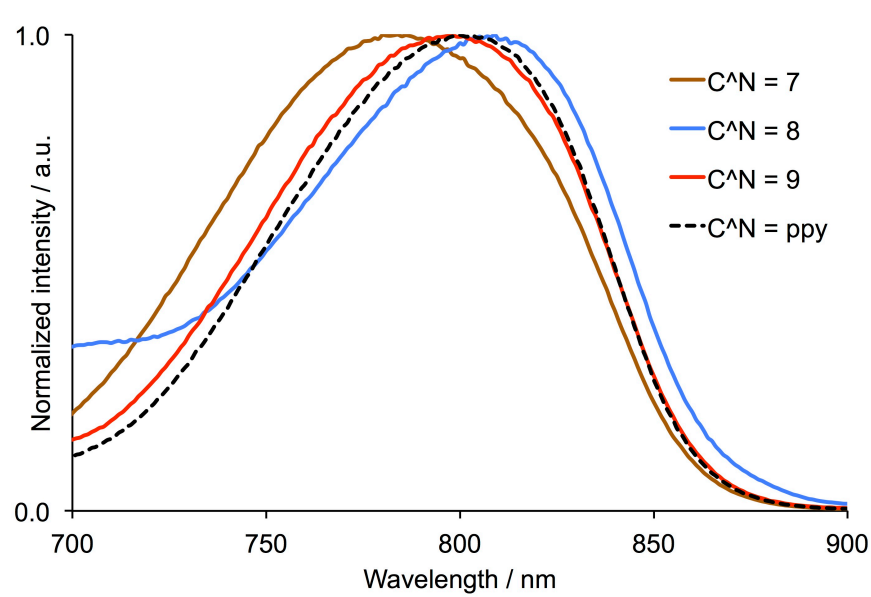

Fig. 13. Normalized solution emission spectra of $\left[R u(b p y)_{2}(7)\right]\left[\mathrm{PF}_{6}\right]$, $\left[\mathrm{Ru}(\mathrm{bpy})_{2}(\mathbf{9})\right]\left[\mathrm{PF}_{6}\right]$ and $\left[\mathrm{Ru}(\mathrm{bpy})_{2}(\mathbf{8})\right]$ compared to $\left[\mathrm{Ru}(\mathrm{bpy})_{2}(\mathrm{ppy})\right]\left[\mathrm{PF}_{6}\right] \quad(\mathrm{MeCN}, 5$ $\times 10^{-5} \mathrm{~mol} \mathrm{dm}^{-3}$ ).

Table 4. Cyclic voltammetric data for $\left[\mathrm{Ru}(\mathrm{bpy})_{2}\left(\mathrm{C}^{\wedge} \mathrm{N}\right)\right]\left[\mathrm{PF}_{6}\right]\left(\mathrm{C}^{\wedge} \mathrm{N}=\mathrm{ppy}, 7\right.$ and 9) and $\left[\mathrm{Ru}(\mathrm{bpy})_{2}(\mathbf{8})\right]$ (degassed $\mathrm{MeCN}$ solutions) with respect to $\mathrm{Fc} / \mathrm{Fc}^{+}$; $0.1 \mathrm{M}\left[{ }^{\mathrm{n}} \mathrm{Bu}_{4} \mathrm{~N}\right]\left[\mathrm{PF}_{6}\right]$ as supporting electrolyte and a scan rate of $0.1 \mathrm{~V} \mathrm{~s}^{-1}$.

\begin{tabular}{|c|c|c|c|c|c|}
\hline Complex & $\begin{array}{c}E_{1 / 2}{ }^{\mathrm{ox}} / \\
\mathrm{V}\end{array}$ & $\begin{array}{c}E_{1 / 2}{ }^{\mathrm{redl}} \\
/ \mathrm{V}\end{array}$ & $\begin{array}{c}E_{1 / 2}{ }^{\mathrm{red} 2} \\
/ \mathrm{V}\end{array}$ & $\begin{array}{c}E_{1 / 2}{ }^{\mathrm{red}} \\
/ \mathrm{V}\end{array}$ & $\begin{array}{c}\Delta E_{1 / 2} / \\
\mathrm{V}\end{array}$ \\
\hline$\left[\mathrm{Ru}(\mathrm{bpy})_{2}(\mathrm{ppy})\right]\left[\mathrm{PF}_{6}\right]$ & 0.09 & -1.96 & -2.22 & & 2.05 \\
\hline$\left[\mathrm{Ru}(\mathrm{bpy})_{2}(\mathbf{7})\right]\left[\mathrm{PF}_{6}\right]$ & 0.13 & -1.95 & -2.14 & -2.40 & 2.08 \\
\hline$\left[\mathrm{Ru}(\mathrm{bpy})_{2}(\mathbf{8})\right]$ & 0.04 & -2.00 & -2.27 & & 2.04 \\
\hline$\left[\mathrm{Ru}(\mathrm{bpy})_{2}(\mathbf{9})\right]\left[\mathrm{PF}_{6}\right]$ & 0.08 & -1.97 & -2.21 & -2.41 & 2.05 \\
\hline
\end{tabular}

Each complex is electrochemically active and cyclic voltammetric data are given in Table 4; Fig. 14 shows the CV of $\left[\mathrm{Ru}(\mathrm{bpy})_{2}(7)\right]\left[\mathrm{PF}_{6}\right]$ as a representative example. Each compound shows a reversible metal-centred process. Compared to a potential of $0.09 \mathrm{~V}$ for the $\mathrm{Ru}^{3+/ 2+}$ couple in $\left[\mathrm{Ru}(\mathrm{bpy})_{2}(\mathrm{ppy})\right]^{+}$, the introduction of the electron-withdrawing ester substituent in $\left[\mathrm{Ru}(\mathrm{bpy})_{2}(7)\right]^{+}$shifts the process to $0.13 \mathrm{~V}$. This trend is consistent with the emission data and the lowering of the HOMO. The similarity of the $E_{1 / 2}{ }^{\text {ox }}$ values for $\left[\mathrm{Ru}(\mathrm{bpy})_{2}(\mathrm{ppy})\right]^{+}$and $\left[\mathrm{Ru}(\mathrm{bpy})_{2}(\mathbf{9})\right]^{+}$(Table 4) is also consistent with the trend in the emission maxima (Table 3 ). The shift to less positive potential for the $\mathrm{Ru}^{3+/ 2+}$ couple on going from $\left[\mathrm{Ru}(\mathrm{bpy})_{2}(\mathrm{ppy})\right]^{+}$to $\left[\mathrm{Ru}(\mathrm{bpy})_{2}(\mathbf{8})\right]$ is consistent with the overall charge on the complex, and is also in line with the red-shift in the emission maximum (Fig. 13). Three reversible ligand-based reduction processes are observed for $\left[\mathrm{Ru}(\mathrm{bpy})_{2}(7)\right]\left[\mathrm{PF}_{6}\right]$ (Fig. $14)$ and $\left[\mathrm{Ru}(\mathrm{bpy})_{2}(\mathbf{9})\right]\left[\mathrm{PF}_{6}\right]$. On going to $\left[\mathrm{Ru}(\mathrm{bpy})_{2}(\mathbf{8})\right]$, the first two reductions are shifted to more negative potential, and only two processes are observed within the electrochemical solvent window.

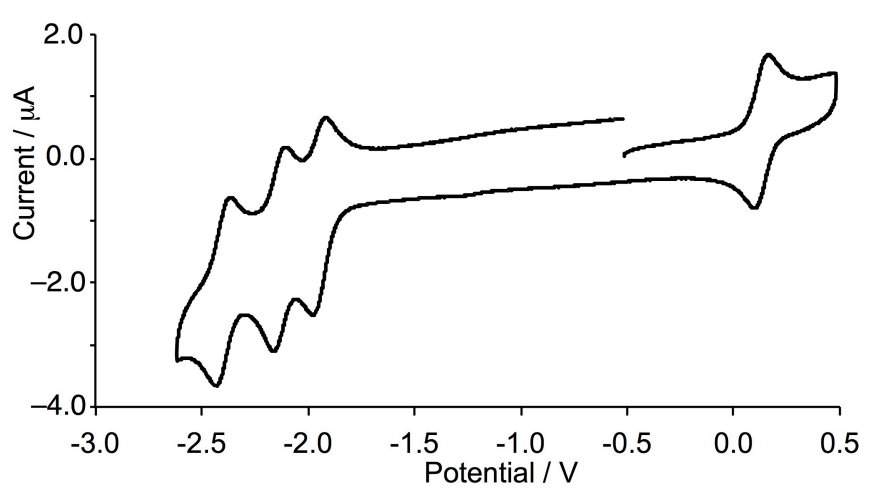

Fig. 14. Cyclic voltammogram of $\left[\mathrm{Ru}(\mathrm{bpy})_{2}(7)\right]\left[\mathrm{PF}_{6}\right]$ (degassed $\mathrm{MeCN}$ solution) with respect to $\mathrm{Fc} / \mathrm{Fc}^{+}$; scan rate $=0.1 \mathrm{~V} \mathrm{~s}^{-1}$.

\section{Conclusions}

A series of $\left[\mathrm{Ru}(\mathrm{bpy})_{2}\left(\mathrm{C}^{\wedge} \mathrm{N}\right)\right]\left[\mathrm{PF}_{6}\right]$ complexes with $\mathrm{C}^{\wedge} \mathrm{N}=\mathbf{1}-\mathbf{7}$ and $\mathbf{9}$ and the zwitter-ion $\left[\mathrm{Ru}(\mathrm{bpy})_{2}(\mathbf{8})\right]$ have been prepared and characterized, and the crystal structures of $\left[\mathrm{Ru}(\mathrm{bpy})_{2}(\mathbf{4})\right]\left[\mathrm{PF}_{6}\right]^{\circ} \mathrm{MeCN}, \quad\left[\mathrm{Ru}(\mathrm{bpy})_{2}(\mathbf{5})\right]\left[\mathrm{PF}_{6}\right] \quad$ and $\left[\mathrm{Ru}(\mathrm{bpy})_{2}(7)\right]\left[\mathrm{PF}_{6}\right]$ determined. $\left[\mathrm{Ru}(\mathrm{bpy})_{2}\left(\mathrm{C}^{\wedge} \mathrm{N}\right)\right]^{+}\left(\mathrm{C}^{\wedge} \mathrm{N}=7\right.$ and 9) and $\left[\mathrm{Ru}(\mathrm{bpy})_{2}(\mathbf{8})\right]$ contain pendant domains that are potential anchors to semiconductor surfaces. The synthesis of the cationic complexes involves the use of $\mathrm{AgPF}_{6}$ to abstract chloride ion from the $c i s-\left[\mathrm{Ru}(\mathrm{bpy})_{2} \mathrm{Cl}_{2}\right]$ precursor; interactions between residual $\mathrm{Ag}^{+}$and the coordinated $\mathrm{C}^{\wedge} \mathrm{N}$ ligand in $\left[\mathrm{Ru}(\mathrm{bpy})_{2}\left(\mathrm{C}^{\wedge} \mathrm{N}\right)\right]^{+}$results in broadened and high-field shifted signals for the cyclometallated ring protons. ${ }^{1} \mathrm{H}$ NMR signals are observed if the freshly prepared $\left[\mathrm{Ru}(\mathrm{bpy})_{2}\left(\mathrm{C}^{\wedge} \mathrm{N}\right)\right]\left[\mathrm{PF}_{6}\right]$ complex is dissolved in $\mathrm{MeCN}$ and the solution left to stand for at least a week followed by filtration through Celite; recrystallized samples also give well resolved NMR spectra. The absorption spectra of $\left[\mathrm{Ru}(\text { bpy })_{2}\left(\mathrm{C}^{\wedge} \mathrm{N}\right)\right]\left[\mathrm{PF}_{6}\right]$ with $\mathrm{C}^{\wedge} \mathrm{N}=\mathbf{1}-$ $\mathbf{6}$ and of $\left[\mathrm{Ru}(\mathrm{bpy})_{2}(\mathbf{8})\right]$ are similar, but the introduction of the anchoring domains in $\left[\mathrm{Ru}(\mathrm{bpy})_{2}\left(\mathrm{C}^{\wedge} \mathrm{N}\right)\right]\left[\mathrm{PF}_{6}\right]$ with $\mathrm{C}^{\wedge} \mathrm{N}=\mathbf{7}$ or 9 enhances the absorption response; the greatest influence on the absorption is seen with the introduction of the diethyl phenylenephosphonate group. Trends in emission and electrochemical behaviours of the complexes are readily interpreted in terms of the influence of the electronic properties of the $\mathrm{C}^{\wedge} \mathrm{N}$ ligand substituents on the energies of the HOMO which is localized on the cyclometalling ligand and the ruthenium centre. This study has allowed us to optimize a synthetic route to the phosphonate ester derivative $\left[\mathrm{Ru}(\mathrm{bpy})_{2}(\mathbf{9})\right]\left[\mathrm{PF}_{6}\right]$ which also exhibits the most promising spectral response among the complexes studied.

\section{Acknowledgements}

We acknowledge the Swiss National Science Foundation, the University of Basel and the European Research Council (Advanced Grant 267816 LiLo) for financial support. D.P.R. would like to thank the Swiss Study Foundation and the Ernst Göhner foundation for support. We thank Dr Daniel Häussinger for assistance with NMR spectroscopic measurements. 


\section{Notes and references}

${ }^{a}$ Department of Chemistry, University of Basel, Spitalstrasse 51, 4056 Basel, Switzerland. email: catherine.housecroft@unibas.ch

${ }^{b}$ Institut für Anorganische und Analytische Chemie, Albert-LudwigsUniversität, Albertstrasse 21, D-79104 Freiburg i. Br., Germany.

$\dagger$ †lectronic Supplementary Information (ESI) available: Infrared spectroscopic data for the $\left[\mathrm{Ru}(\mathrm{bpy})_{2}\left(\mathrm{C}^{\wedge} \mathrm{N}\right)\right]\left[\mathrm{PF}_{6}\right]$ complexes and $\left[\mathrm{Ru}(\mathrm{bpy})_{2}(\mathbf{8})\right]$; Figs. S1-S10: ${ }^{1} \mathrm{H}$ NMR spectra of the complexes. See DOI: $10.1039 / \mathrm{b} 000000 \mathrm{x} /$

1 E. C. Constable and J. M. Holmes, J. Organomet. Chem., 1986, 301, 203.

2 P. Reveco, W. R. Cherry, J. Medley, A. Garber, R. J. Gale and J. Selbin, Inorg. Chem., 1986, 25, 1842.

3 A. Colombo, C. Dragonetti, A. Valore, C. Coluccini, N. Manfredi and A. Abbotto, Polyhedron, 2014, doi.org/10.1016/j.poly.2014.04.048.

4 K. C. D. Robson, P. G. Bomben and C. P. Berlinguette, Dalton Trans., 2012, 41, 7814.

5 P. G. Bomben, K. C. D. Robson, B. D. Koivisto and C. P. Berlinguette, Coord. Chem. Rev., 2012, 256, 1438.

6 S. H. Wadman, J. M. Kroon, K. Bakker, M. Lutz, A. L. Spek, G. P. M. van Klinka and G. van Koten, Chem. Commun., 2007, 1907.

7 T. Bessho, E. Yoneda, J.-H. Yum, M. Guglielmi, I. Tavernelli, H. Imai, U. Rothlisberger, M. K. Nazeeruddin and M. Grätzel, J. Am. Chem. Soc., 2009, 131, 5930.

8 P. G. Bomben, K. C. D. Robson, P. A. Sedach and C. P. Berlinguette, Inorg. Chem., 2009, 48, 9631.

9 R. D. Costa, E. Ortí, H. J. Bolink, F. Monti, G. Accorsi and N. Armaroli, Angew. Chem. Int. Ed., 2012, 51, 8178.

10 See for example: E. Yoneda, M. K. Nazeeruddin and M. Graetzel, $J$. Photopolymer Sci. Technol, 2012, 25, 175; T. Funaki, N. OnozawaKomatsuzaki, K. Kasuga, K. Sayama and H. Sugihara, Inorg. Chem. Comm., 2013, 35, 281; V. A. Grinberg, A. V. Medved'ko, V. V. Emets, S. A. Kuzeev, S. A. Kozyukhin, A. E. Baranchikov, V. K. Ivanov, V. N. Andreev and E. A. Niznikovskii, Russ. J. Electrochem., 2014, 50, 503; T. Funaki, N. Koumura and K. Sayama, Chem. Lett., 2013, 42, 1371; M. He, Z. Ji, Z. Huang and Y. Wu, J. Phys. Chem. C, 2014, doi.org/10.1021/jp4117694.

11 P. G. Bomben, K. D. Thériault and C. P. Berlinguette, Eur. J. Inorg. Chem., 2011, 1806.
12 S. Soman, Y. Xie and T. W. Hamann, Polyhedron, 2014, doi.org/10.1016/j.poly.2014.05.061.

13 J. He, H. Lindström, A. Hagfeldt and S.-E. Lindquist, Solar Ener. Mater. Solar Cells, 2000, 62, 265.

14 Z. Ji, G. Natu, Z. Huang, O. Kokhan, X. Zhang and Y. Wu, J. Phys. Chem. C, 2012, 116, 16854.

15 Z. Ji and Y. Wu, J. Phys. Chem. C, 2013, 117, 18315.

16 Y. Pellegrin, L. Le Pleux, E. Blart, A. Renaud, B. Chavillon, N. Szuwarski, M. Boujtita, L. Cario, S. Jobic, D. Jacquemin and F. Odobel, J. Photochem. Photobiol. A, 2011, 219, 235.

17 P. Péchy, F. P. Rotzinger, Md. K. Nazeeruddin, O. Kohle, S. M. Zakeeruddin, R. Humphry-Baker and M. Grätzel, J. Chem. Soc., Chem. Commun., 1995, 65.

18 S. M. Zakeeruddin, M. K. Nazeeruddin, P. Pechy, F. P. Rotzinger, R. Humphry-Baker, K. Kalyanasundaram, M. Grätzel, V. Shklover and T. Haibach, Inorg. Chem., 1997, 36, 5937.

19 D. K. Zhong, S. Zhao, D. E. Polyansky and E. Fujita, J. Catal., 2013, 307, 140 .

20 F. C. Krebs and M. Biancardo, Solar Ener. Mater. Solar Cells, 2006, 90, 142.

21 E. C. Constable, C. E. Housecroft, M. Šmídková and J. A. Zampese, Can. J. Chem., 2014, 92, 724.

22 G. Guerrero, P. H. Mutin, A. Vioux, Chem. Mater., 2001, 13, 4367.

23 G. Guerrero, P. H. Mutin, E. Framery and A. Vioux, New J. Chem., 2008, 32, 1519.

24 C. Liu, Q. Ni, P. Hu and J. Qiu, Org. Biomol. Chem., 2011, 9, 1054.

25 C. Liu and W. Yang, Chem. Commun., 2009, 6267.

26 J. Wu, D. Zhang, L. Chen, J. Li, J. Wang, C. Ning, N. Yu, F. Zhao, D. Chen, X. Chen, K. Chen, H. Jiang, H. Liu and D. Liu, J. Med. Chem., 2013, 56, 761.

27 E. C. Constable, C. D. Ertl, C. E. Housecroft and J. A. Zampese, Dalton Trans., 2014, 43, 5343.

28 Y. Li, Y. Liu, and M. Zhou, Dalton Trans., 2012, 41, 2582.

29 Bruker Analytical X-ray Systems, Inc., 2006, APEX2, version 2 User Manual, M86-E01078, Madison, WI.

30 P. W. Betteridge, J. R. Carruthers, R. I. Cooper, K. Prout and D. J. Watkin, J. Appl. Cryst., 2003, 36, 1487.

31 G. M. Sheldrick, Acta Crystallogr., Sect. A, 2008, 64, 112.

32 I. J. Bruno, J. C. Cole, P. R. Edgington, M. K. Kessler, C. F. Macrae, P. McCabe, J. Pearson and R. Taylor, Acta Crystallogr., Sect. B 2002, 58, 389.

33 C. F. Macrae, I. J. Bruno, J. A. Chisholm, P. R. Edgington, P. McCabe, E. Pidcock, L. Rodriguez-Monge, R. Taylor, J. van de Streek and P. A. Wood, J. Appl. Cryst., 2008, 41, 466. 
34 A. L. Spek, Acta Crystallogr., Sect. D, 2009, 65, 148.

35 E. C. Constable, A. Hernandez Redondo, C. E. Housecroft, M. Neuburger and S. Schaffner, Dalton Trans., 2009, 6634.

36 B. Bozic-Weber, S. Y. Brauchli, E. C. Constable, S. O. Fürer, C. E. Housecroft, F. J. Malzner, I. A. Wright and J. A. Zampese, Dalton Trans., 2013, 42, 12293.

37 M. Querol, B. Bozic, N. Salluce, and P. Belser, Polyhedron, 2003, 22, 655 .

38 M. Montalti, S. Wadhwa, W. Y. Kim, R. A. Kipp, and R. H. Schmehl, Inorg. Chem., 2000, 39, 76.

39 V. Penicaud, F. Odobel and B. Bujoli, Tetrahedron Lett., 1998, 39, 3689.

40 W. M. Ward, B. H. Farnum, M. Siegler and G. J. Meyer, J. Phys. Chem. A, 2013, 117, 8883 .

41 G. E. Schneider, H. J. Bolink, E. C. Constable, C. D. Ertl, C. E. Housecroft, A. Pertegas, J. A. Zampese, A. Kanitz, F. Kessler and S. B. Meier, Dalton Trans., 2014, 43, 1961.

42 See for example: Y. Habata, M. Ikeda, S. Yamada, H. Takahashi, S. Ueno, T. Suzuki and S. Kuwahara, Org. Lett., 2012, 14, 4576; Y. Habata, A. Taniguchi, M. Ikeda, T. Hiraoka, N. Matsuyama, S. Otsuka and S. Kuwahara, Inorg. Chem., 2013, 52, 2542; J. Kang, M. Choi, J. Y. Kwon, E. Y. Lee and J. Yoon, J. Org. Chem., 2002, 67, 4384.

43 J. C. Dobson, B. P. Sullivan, P. Doppelt and T. J. Meyer, Inorg. Chem., 1988, 27, 3863.

44 F. H. Allen, Acta Crystallogr., Sect. B, 2002, 58, 380.

45 M. Brissard, M. Gruselle, B. Malézieux, R. Thouvenot, C. GuyardDuhayon and O. Convert, Eur. J. Inorg. Chem., 2001, 2001, 1745.

46 E. V. Ivanova, I. V. Kurnikov, A. Fischer, L. Alexandrova and A. D. Ryabov, J. Mol. Catal. B, 2006, 41, 110.

47 M. L. Muro-Small, J. E. Yarnell, C. E. McCusker and F. N. Castellano, Eur. J. Inorg. Chem., 2012, 2012, 4004.

48 G. R. Desiraju and T. Steiner, The Weak Hydrogen Bond, Oxford University Press, 1999, Chapter 2. 